\title{
A REFLECTION ON A BONE TISSUE ENGINEERING DESIGN AND COMMERCIALISATION PROJECT
}

\section{BY}

\section{THOMAS SOBIECKI}

\author{
A thesis \\ submitted to the Victoria University of Wellington \\ in partial fulfilment of the requirements for the degree of \\ Master of Advanced Technology Enterprise
}

Victoria University of Wellington 


\begin{abstract}
A commercialisation project centred round a material called synthetic nacre was undertaken as a team as part of the 2014 Masters of Advanced Technology Enterprise (MATE) programme. There were multiple goals of: examining the individual role within the group, from an engineering discipline (mechatronics), and what it means for building successful teams; finding and developing the material for a market application, in this case the niche of biodegradable osteoconductive load bearing biomaterials for orthopaedic implants; and reflecting on the personal contribution to the commercialisation process and how successful it was.

The role of an engineer to solve problems was proposed and found to be partially true in this case; additionally a secondary role in communicating technical information coherently was also apparent and important to the enterprise development. An adaptive biomaterial design concept and specification for the target application was formed using the literature and extrapolating where there was no resolution or gaps in the research. The influence of mechatronics has been established on the decision making process and direction of the commercialisation project. The design process was incomplete and therefore the enterprise develop was unsuccessful as it has not been validated by going through a full design, test, evaluate cycle. The goals of the course environment and the team building approach further reinforces this belief.
\end{abstract}




\section{ACKNOWLEDGMENTS}

Many thanks to the following:

Natasha Evans who helped and provided guidance through the project on many of the chemistry aspects unfamiliar to me. Especially her patience in instructing me, a person without any tertiary level chemistry at all, through the multiple process method to fabricate synthetic nacre - any errors within are of my own creation.

Professor Kathryn McGrath for providing such an interesting material and project and giving us free reign to attempt to find a market application for it.

My fellow bone project team members: Christina Houlihan and Michael Mettrick for holding in there through the highs and lows.

Melissa Yiannoutsos and Jennifer Anderson from Kerasi Limited who guided as through the commercialisation aspects of the project as it became increasing clear how early stage it was.

My supervisor Ramesh Rayudu for taking a flexible and understanding approach to such a new and changing course.

Thomas Sobiecki

Wellington, February 2014 


\section{CONTENTS}

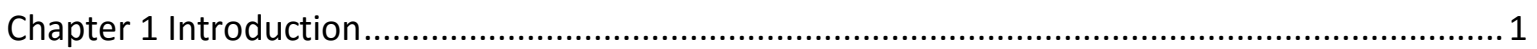

Master of Advanced Technology Enterprise (MATE) ........................................................... 1

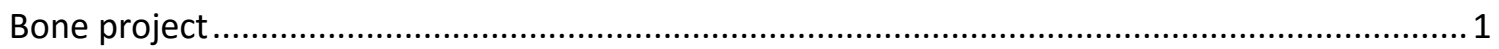

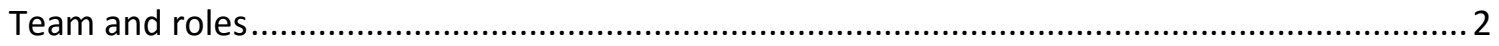

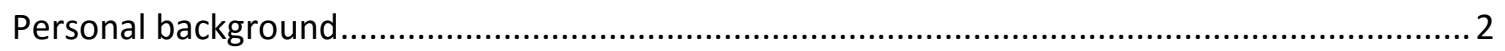

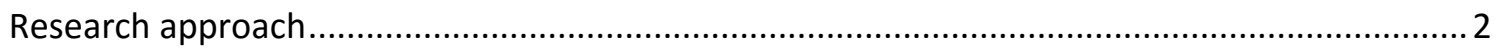

Chapter 2 Synthetic Nacre - Understanding the current status...............................................

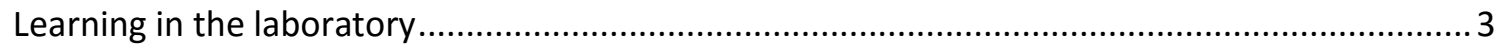

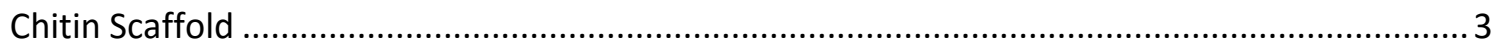

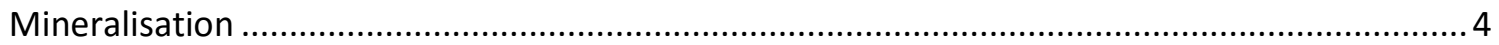

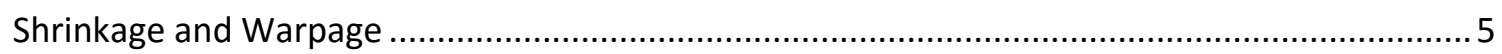

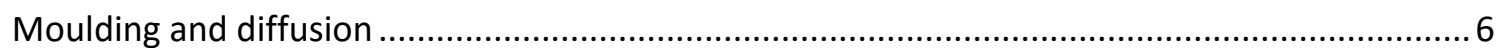

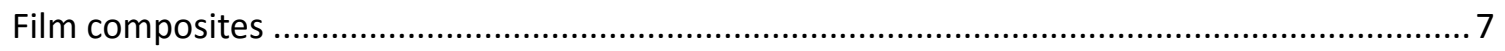

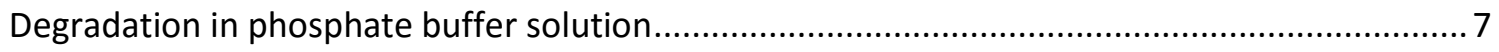

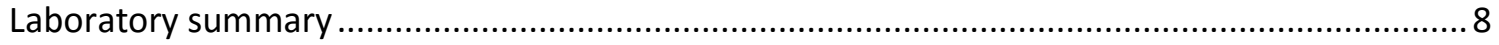

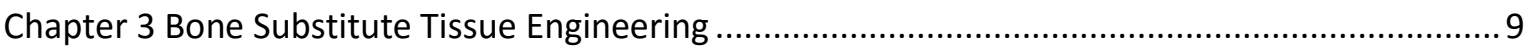

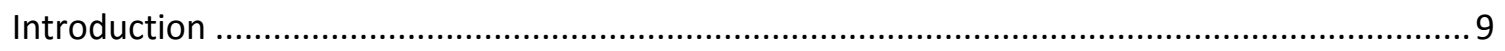

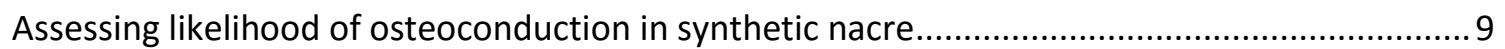

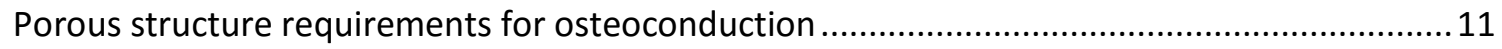

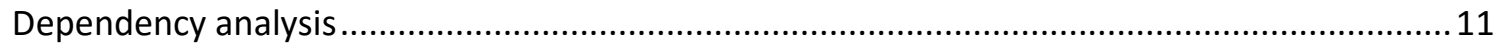

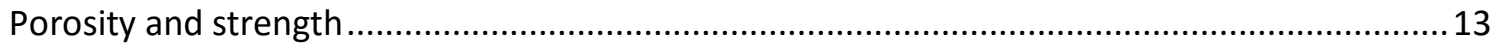

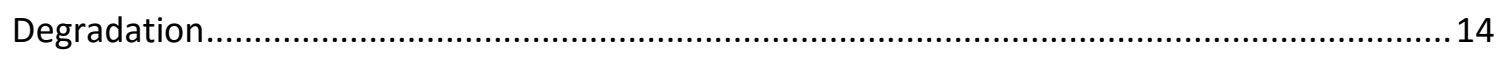

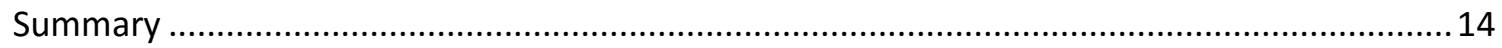

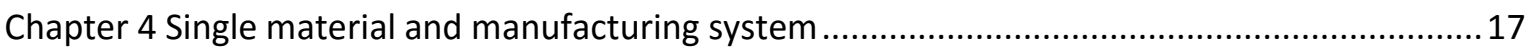

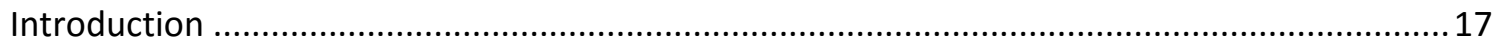

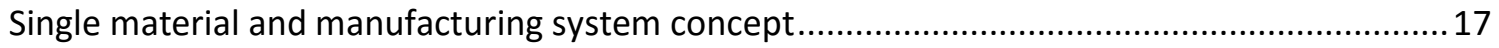

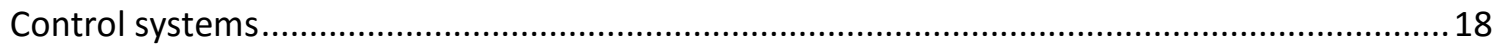




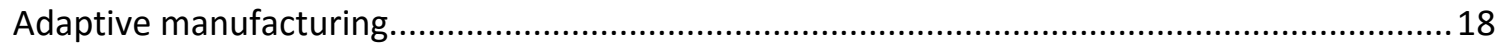

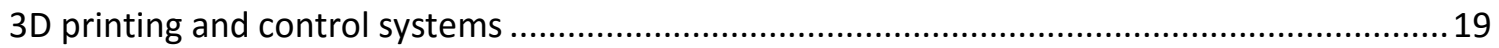

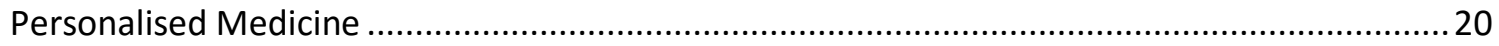

Toyota Product Development System (Lean Product Development) ........................................... 21

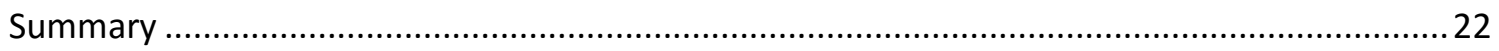

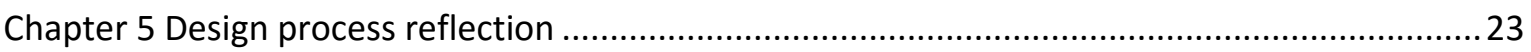

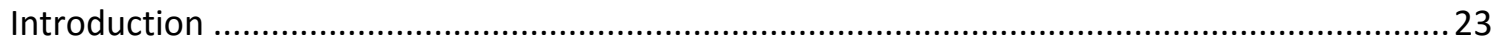

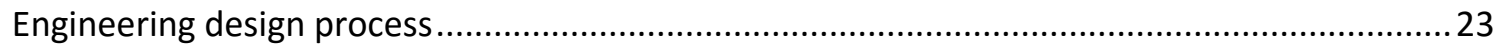

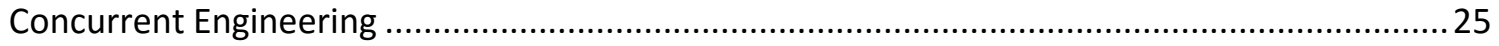

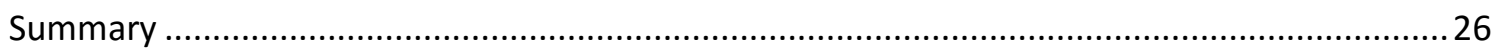

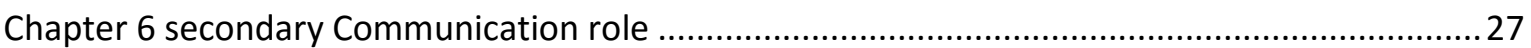

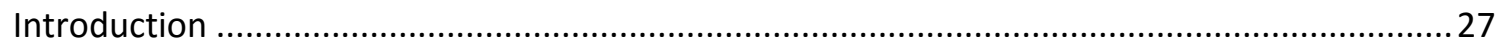

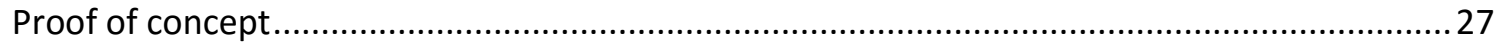

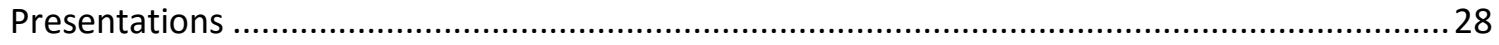

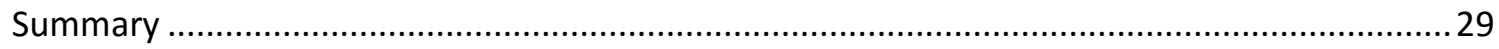

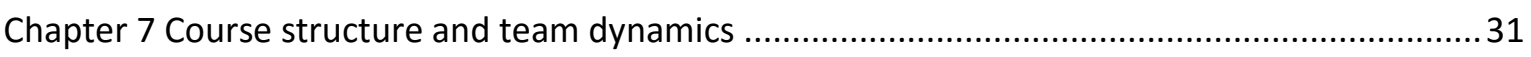

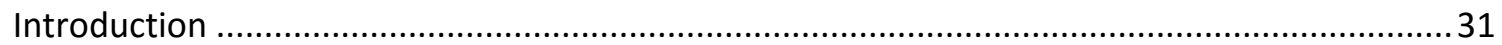

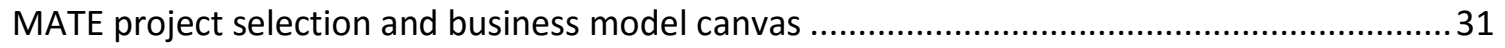

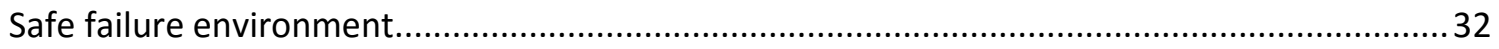

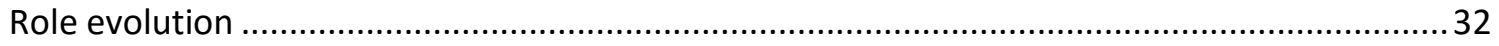

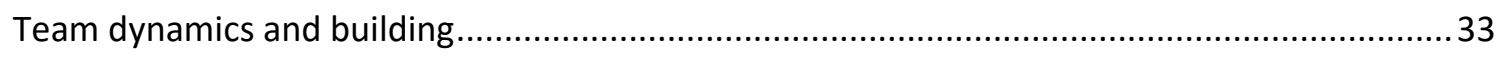

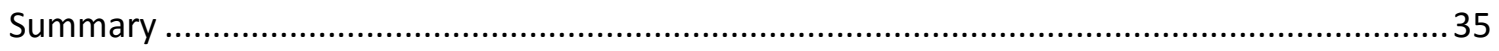

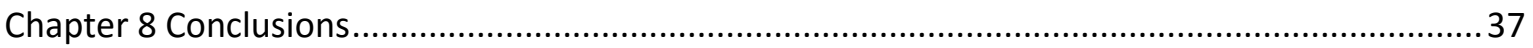

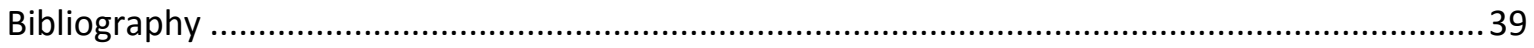




\section{LIST OF FIGURES}

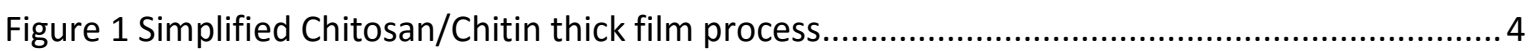

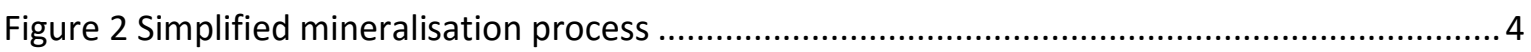

Figure 3 Non-uniform drying of coating edge; adapted from Radhakrishnan (2006) ....................... 5

Figure 4 a) SEM pre-PBS showing aragonite disc b) SEM post-PBS showing calcium phosphate

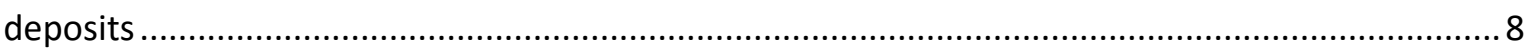

Figure 5 Dependency analysis between synthetic nacre and osteoconduction ............................. 12

Figure 6 Dependency analysis for osteoconduction ................................................................... 12

Figure 7 Conceptual relationship between porosity, strength, and degradation characteristics. Actual relationships will are unknown but likely non-linear. Shows potential optimal regions for

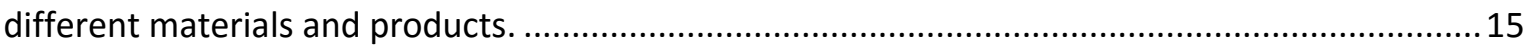

Figure 8 Adaptive manufacturing feedback system ...................................................................... 19

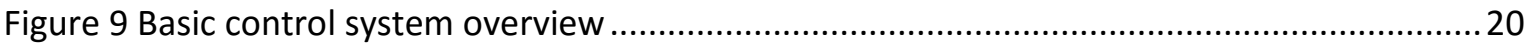

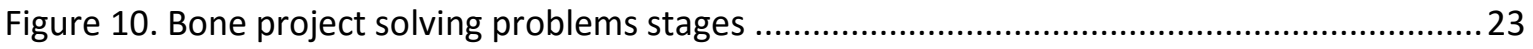

Figure 11. An engineering design process; adapted from Avramenko (2008)............................... 24

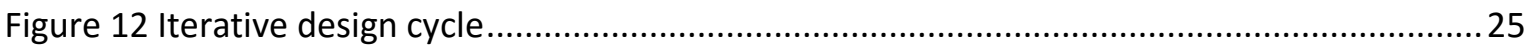

Figure 13 Artistic impressions of the different porous structures: isolated, interconnected, and sparse pores. 


\section{CHAPTER 1 INTRODUCTION}

\section{Master of Advanced Technology Enterprise (MATE)}

An important part of improving the New Zealand economy is developing advanced technology enterprises. Building teams and understanding the roles within them is a key aspect of successfully start-ups and commercialisation of the research and technology being developed within the country's higher education centres. In its second year running the MATE programme is structured around a practical team based science commercialisation project and business case study. The focus is on examining the individual roles and disciplines within a team as advanced technology, for example a piece of research from the Victoria University of Wellington (VUW), is developed towards a market application. Reflection on the practical components from personal perspectives and disciplines is used as a tool to support the formation of individual dissertations. Though restricted to a discipline the enterprise development and its evolution is allowed to influence the direction of the research.

\section{Bone project}

In 2014 there were three projects provided to the students via "project champions"; nominally individual researchers sponsoring their work for the students of the programme to select and form teams around. One of these, a repeated entry from the previous year based on a novel material developed by Professor Kathryn McGrath and her research group, dubbed the bone project formed starting point for developing the business case study.

The novel material was described as synthetic nacre or mother-of-pearl which also forms the semiiridescent inside lining of mollusc shell. A combination of the natural renewable polymer chitin and calcium carbonate formed in the crystal structure aragonite (Munro \& McGrath, 2012) an application identified for the material was as a bone substitute in orthopaedic surgery. The previous year's team had been provided a very narrow target market to investigate, dental void fillers to be used for animals with teeth removed, which was quickly shown to be of no interest since for animals there is a lower standard of care. Importantly however they learnt that it would be essential for synthetic nacre to have a feature called osteoconduction (Townend, 2014, p. 5a). While no target market was suggested for the current year medical, and particularly orthopaedics, was still the route taken (due to the high cost of the raw materials) and this information provided the starting point for the project. Additional market research eventually helped refine the synthetic nacre target application to a biomaterial for biodegradable osteoconductive load bearing orthopaedic implants. 
It was understood from the beginning of the project that due to being in the medical space there would be no possibility that a market ready product would be developed by the end of the year; in fact later estimates suggested it may take over 10 years. As such milestones were set as goals to progress the commercialisation towards (particularly to reach a basic proof of concept stage which was not achieved) and used to measure success. The end result of enterprise development, in part, was a target specification to obtain osteoconduction and a concept for a single material and manufacturing system. This concept could allow fabricating the synthetic nacre biomaterial with tailored properties to support multiple target applications and, in the future, personalised implants.

\section{Team and roles}

The project was chosen and what role within the team formed around that was dependent on each member's discipline and personal background. Aside from myself the bone project team included Christina Houlihan, whose contributions can be found in the intellectual property, regulatory, and some biological sections of the case study, and Michael Mettrick, whose contributions can be found in the market, strategy, and competitor analysis. The author contributed to the technical aspects and product concept design found in the biomaterial, scale up, and proof of concept sections.

\section{Personal background}

A previous bachelors in mechatronics engineering from Massey University, Palmerston North, provided a multidisciplinary technical base including soft skills such as product development for the course and the bone project.

\section{Research approach}

The research direction is intended to be influenced by the development and evolution of the bone project enterprise and individual discipline; as such there are three items examined: 1) the role of an engineer within a team to solve problems; 2 ) the ideal design concept for a biodegradable load bearing osteoconductive bone biomaterial; and 3) the engineering design process followed and its success.

The first sections discuss aspects of the technical product concept development around biodegradable osteoconductive load bearing biomaterials for orthopaedic implants and its supporting manufacturing system concept. Following is a reflection on the overall design process, including a discussion on how mechatronic skills supported the direction of the bone project, proposing it was unsuccessful. Included is a secondary role that hints at the importance of communication. Lastly the overall course and team environment reinforces the argument and raises shortcomings regarding the development of a successful team. 


\section{CHAPTER 2 SYNTHETIC NACRE - UNDERSTANDING THE CURRENT STATUS}

\section{Learning in the laboratory}

An initial task was to understand the current status and capabilities of the synthetic nacre material developed by Professor Kathryn McGrath and the VUW research group. The original goal was to learn the chemical fabrication process and transfer to the bone project team the capability to manufacture specimens for mechanical strength testing without using valuable researcher time.

Developing an understanding of the process and current status of the synthetic nacre material proved crucial; it identified a number of issues to overcome and changed the direction of the commercialisation project in a number of ways. This section focuses on work completed in the laboratory under the guidance of Dr Natasha Evans and importantly the observations of and discussions with the individual researchers regarding their efforts to manipulate the material which affected the direction of the bone project's development.

The capabilities the VUW research group had previously demonstrated included fabricating the synthetic nacre material in different forms as thick films, microspheres, and small 3D printed hollow cylinders or rings. As well as 3D printing and simply drying the material in petri dishes (for thick films) they had briefly experimented with foaming (forming a highly porous but weak material), incorporating magnesium with the calcium carbonate, and throughout much of the year were attempting to develop a method to manufacture specimens for strength testing.

\section{Chitin Scaffold}

Chitosan/Chitin is a natural polymer that is soluble in (acidic) solution depending on its chemical structure, i.e. degree of acetylation, and is either referred to as chitosan or chitin. The separation is indistinct, acting as a scale, where the less acetylated the "more chitosan" it is and the easier it is to dissolve; vice versa for chitin (Aranaz et al., 2009). An approximate definition for chitosan is where the degree of acetylation in chitin has been reduced to less than $50 \%$ (Rinaudo, 2006).

A method to produce synthetic nacre films was learnt under instruction which has been separated into two stages with the first being the fabrication of the chitin polymer scaffold (Figure 1). This produces chitin thick films (approximately between 100 and 500 $\mu$ m thick) that are then mineralised later to form synthetic nacre - an immediate problem noticed was warping, the films deforming away from being flat, and shrinkage as the material dried. These problems, previously not known to the project team but understood by the researchers, were discussed and explanations found or proposed where possible. 


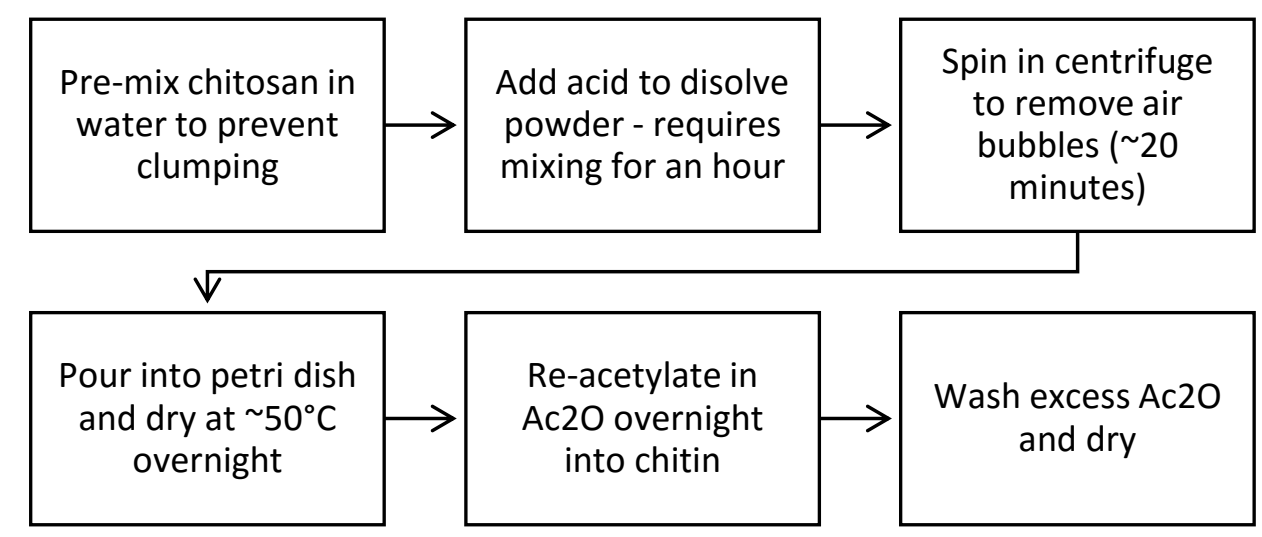

Figure 1 Simplified Chitosan/Chitin thick film process

\section{Mineralisation}

The mineralisation process (Figure 2 ) is where the calcium carbonate in the form of aragonite is integrated into the chitin scaffold - not only on the surface but defused to a depth into the polymer. It is a three stage process starting with embedding calcium ions and then carbonate ions to form the initial aragonite crystal structure. The sodium bicarbonate also has the effect of crosslinking the polymer chains curing the material - essentially lessening its ability to absorb water and expand/shrink. The final five day bath in calcium carbonate solution reinforces the mineralisation already present increasing the ratio of calcium carbonate to scaffold.

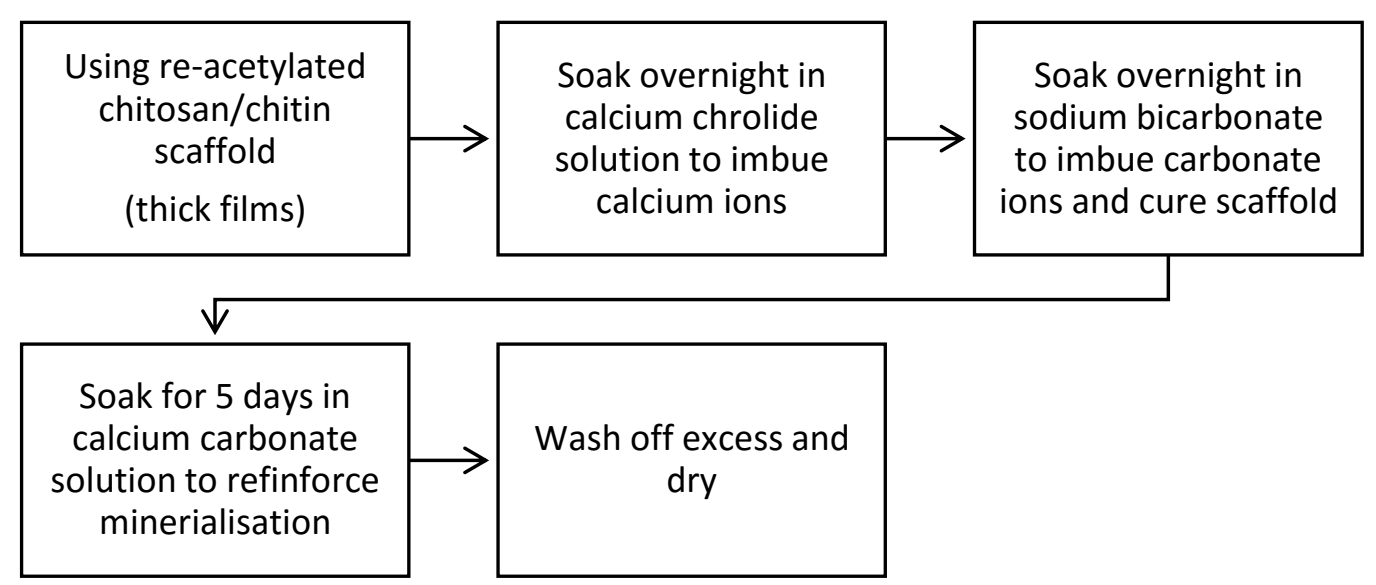

Figure 2 Simplified mineralisation process

While an effort has been made to make sure this description is accurate without having a strong background in chemistry there was confusion at times. For example the water absorption properties are affected by both re-acetylation, which increasing the hydrophobicity of the material, and cross-linking which bindings the polymer chains closer together. It was during the discussions with the VUW research group to clarify this relationship that it was revealed how variable or unfixed 
the process still was in their eyes. The chemicals used could be changed to stronger or weaker types, additional steps could be inserted (such as base treatments to make the chitosan scaffold insoluble), and depending on the fabrication method (thick films, 3D printing, beads) different process steps were used.

\section{Shrinkage and Warpage}

The chitosan/chitin scaffolds solidify via drying. This made previous experience, from mechatronics, with injection moulding less useful during the bone project since the synthetic plastics used solidify via a thermal process. Water content in plastic is an issue but is typically dealt with by drying the plastic granules before moulding. Nylon is considered to have a high water content at $1-2 \%$ or $5-$ $8 \%$ when immersed (Sepe, 2013). These are very low percentages when compared to chitosan/chitin, a hydrogel, which when absorbing water can become as much as $60 \%$ water (Ostrowska-Czubenko, Pieróg, \& Gierszewska-Drużyńska, 2013).

Nevertheless moulding traditional plastic components does lead to shrinkage and warpage due to, among many reasons, non-uniform thermal solidification (Zheng, Tanner, \& Fan, 2011). The much higher shrinkage rates in chitin/chitosan was one basis that was used to suggest that the excessive dimensional instability scaffolds is a key issue to overcome and enable the fabrication of synthetic nacre biomaterial implants.

Solidification via drying appears more relevant in literature to coatings which, as the chitosan/chitin scaffold process is used to create thick films that form over a plastic substrate surface (the petri dish), help adequately explain the distortions encountered during the bone project. For instance the edges of the chitin films fabricated by the VUW research group may dry in a manner similar to (Figure 3) which illustrates how non-uniform drying occurs at the edge of a coating; either thickening or thinning the edge (Radhakrishnan, 2006).

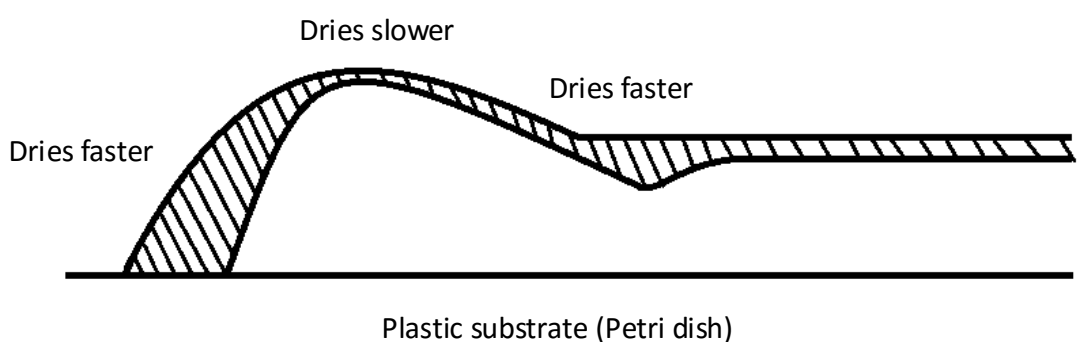

Figure 3 Non-uniform drying of coating edge; adapted from Radhakrishnan (2006) 
Methods exist that help reduce the distortion of the chitosan films. A basic example is washing off the excess Ac2O (Figure 1) with water forms ascetic acid which is the acid used to dissolve the original chitosan powder. Replacing the water with methanol (the chemical Ac2O is mixed with to form a solution for re-acetylation) would remove the excess without forming the acid by-product. It was stated that this was an obvious change to the process that could be made but the focus of their research had been on the chemical structure and its effects rather than producing consistent undistorted films. This confirmed the need to transfer the capabilities across as, justifiably so, the VUW research group and the bone project team had different goals and priorities.

One current procedure, used by the VUW research group to mitigate distortion, is to remove the warped edges before the re-acetylation process. This appeared to have limited success and it may be that, since re-acetylation may act almost as a re-solidification, the repeated chemical processes exaggerates any small non-uniformity that might normally only cause a slight defect after a single process. Additionally during the mineralisation the curing process (Figure 2) will cause further distortion. Cross-linking the polymer chains binds them together, restricting the scaffold's ability to absorb water, and is the final process that stabilises the material.

\section{Moulding and diffusion}

During the time in the laboratory while learning the process to fabricate the synthetic nacre thick films it was possible to observe and discuss with the individual researchers a number of their experiments; amongst these was an attempt to manufacture specimens for strength testing using a moulding technique. The problems encountered included handling steps such as difficulty removing the moulded chitosan scaffold from the die (which was resolved by re-ordering the die in Teflon) and the recurring issue with the expansion and shrinkage of the chitosan material creating unusable warped specimens.

In discussing these problems with the VUW research group a number of (limited) recommendations were provided based on an understanding of moulding processes. These included drafting the mould to ease removing the part but excluded (at the time less assured) opinions on how problematic the extensive shrinkage would be; despite knowing the much lower percentages in thermoplastics are a challenge. Advising that a moulding process would not be suitable could have saved time and effort and perhaps allowed for a successful 3D structure manufacturing technique to be developed (for example by resolving the issues with the 3D printer).

The experiments were beneficial for the bone project team however as it was during these that the issue with the limits of diffusion were observed. The limit of diffusion here refers to depth of polymer scaffold mineralisation. It was initially believed that the mineralisation depth was less than 
$3 \mathrm{~mm}$ but it was revealed to be closer to 0.5 to $1 \mathrm{~mm}$. Additionally not only was the mineralisation restricted but also the re-acetylation process. For the larger thickness of chitosan in the moulded specimens the chemical reaction appeared to be non-uniform; it was suggested that a chemical reaction "front" trapped solution and by-products within the centre of the chitosan material as the outside was transformed into chitin. The limitation on wall thickness turned out not to be an issue in the long term as the porous structure design discussed later (p. 11) required walls of similar dimensions; nullifying the issue.

\section{Film composites}

As the VUW research group was focused on the chemical structure goals very little had been investigated for dimensional stability or building structures. For strength testing, as the 3D printer was not working, a number of simple experiments were completed where the re-acetylation process was used to "glue" the chitosan films together (while transforming it into chitin). A double layer of chitosan thick film and a curled hollow cylinder were constructed; the initial intention was to test whether this technique could be used to create cored composite specimens (an everyday example of a cored sandwich composite is corrugated cardboard). For the chitosan/chitin scaffold this structure would have solved the diffusion issues by having a large porous structure (too large when regarding the final biomaterial design specifications developed) and allow compression strength testing to be performed. Due to the issues with distortion mentioned above (p. 5) this did not progress.

In relation to these simple composite structures it is possible to use chitosan/chitin fibres to selfreinforce the synthetic nacre material. Self-reinforcing is where the orientation of fibres within a body, or matrix, of the same material provide additional strength in a particular direction (Morgan, Weager, Hare, Bishop, \& Smith, 2009) (in exchange for weakness in another direction). Achieving high strength was a concern and this approach, already demonstrated in PLA based materials (Charles, Kramer, Shaw, Olson, \& Wei, 2013; Törmälä, 1992), is one approach to mitigate it. Additionally as it is possible to produce chitosan fibres using electrospinning (Sun, 2011) there is potential for fabric weaves or cloth. Implants built from this, in a similar manner as reinforcing fibres, could provide increased mechanical strength.

\section{Degradation in phosphate buffer solution}

Among the competing biomaterials a common test was immersion in phosphate buffer solution (PBS) at $37^{\circ} \mathrm{C}$ to mimic, to a limited extent, the environment within the human body. It was known that this would not degrade the chitosan/chitin scaffold, which requires an enzyme to be broken down, but would affect the calcium carbonate component of the composite material. As the cost 
was minor a number of thick film samples were used to recreate the test weighing dried samples at set points over a number of days. Unexpectedly, instead of the aragonite dissolving into the surrounding solution, the samples gained weight - a surface reaction was occurring that transformed the surface calcium carbonate into calcium phosphate (Ni, 2003). This was confirmed with scanning electron microscopy for the synthetic nacre thick film samples (Figure 4).

a)

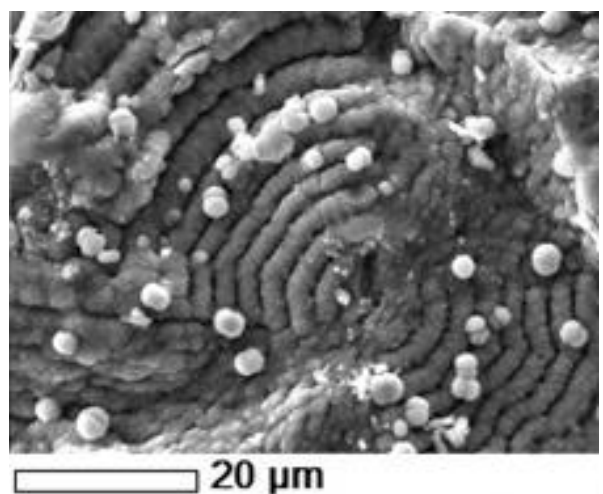

b)

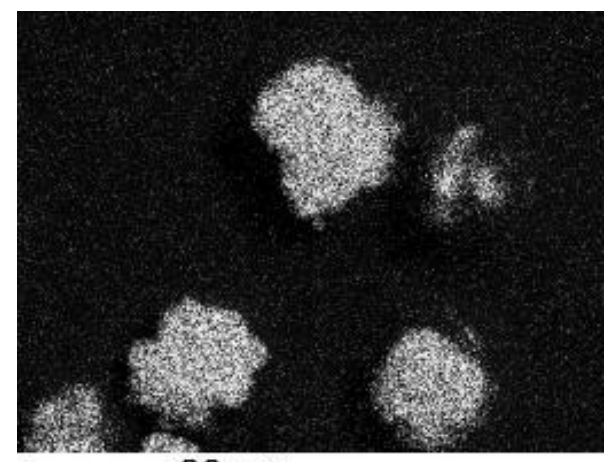

$30 \mu \mathrm{m}$

Figure 4 a) SEM pre-PBS showing aragonite disc b) SEM post-PBS showing calcium phosphate deposits

Later it was found that this may be an advantage since the biomaterial implants may form a strong chemical binding, or rather a multiphase interface, between the synthetic nacre and the calcium phosphate in the form of hydroxyapatite (bone); this effect has been confirmed in sheep using the nacre from marine life (Atlan et al., 1999).

\section{Laboratory summary}

By working in the laboratory a large number of issues were identified with the synthetic nacre material and its fabrication process - highlighting the importance of communication between the project team and the VUW research group and individual researchers. Firstly the natural polymer scaffold which, due to being chitosan/chitin and a hydrogel, warped and distorted from the desired specimen shape. Secondly the limits of diffusion limited the thickness of the synthetic nacre material to less than $0.5 \mathrm{~mm}$. A number of potential avenues were identified to help with the warping, diffusion limitations, and mechanical strength.

The flexibility of the chemical process and its early development stage was now known resulting in two things occurring: 1) the technical capabilities of the synthetic nacre material became a variable target 2) the synthetic nacre material was not ready for commercialisation leading to the role responsibilities evolving into the development of specific targets for synthetic nacre to achieve. 


\section{CHAPTER 3 BONE SUBSTITUTE TISSUE ENGINEERING}

\section{Introduction}

The chosen niche application for the synthetic nacre was as a biodegradable osteoconductive load bearing biomaterial for orthopaedic implants. Biodegradable refers to the ability of the material to be absorbed, or degraded, over time within the human body; its major advantage is that there is no implant remaining, which either needs surgery to remove or is left in long term, after the healing process. Osteoconduction is where natural bone grows through the implant material; replacing it as it degrades. Load bearing refers to having high strength that it can be used in fixation where large forces are applied, for instance breakage repairs in the lower limbs.

The previous year's team identified osteoconduction as a key capability the synthetic nacre. Originally this was not part of the author's role, being at first glance biological, but evolved to include it as the multidisciplinary nature of tissue engineering became apparent; particularly the pre-eminence of the interconnected macro porous structure in combination with micro-structure design features. A large proportion of this task, much simplified, resulted in the biomaterial target specifications within the appendix.

Building upon this the literature around bone biomaterials was reviewed, in combination with dependency analysis, to assess the likelihood that the synthetic nacre material would support osteoconduction. The counteracting nature of porosity and strength is examined leading to the necessity for (not just an option of) a 3D printing fabrication process.

\section{Assessing likelihood of osteoconduction in synthetic nacre}

Synthetic nacre is a composite or hybrid material of calcium carbonate (in aragonite form) and chitin; the natural form also has trace elements, proteins, and other large molecules. The lack of proteins and contaminants, as suggested by the VUW research group, makes the material suitable for medical purposes since they can cause an immune response - otherwise significantly cheaper discarded shells from, for example, mussel farms could be used. Despite the differences the medical literature was used to help provide confidence that synthetic nacre would be biocompatible and safe to use in vivo and support bone healing. While nacre has been used in humans before ( Atlan, Balmain, Berland, Vidal, \& Lopez, 1997) and supported bone growth it was also suggested that this may be due to the proteins (Pereira Mouriès, Almeida, Milet, Berland, \& Lopez, 2002) which have some osteogenic properties. Osteogenesis can be described as spontaneous bone growth; where in osteoconduction ingrowth (via the surface of the porous scaffold) occurs from surrounding bone (Shahgoli \& Levine, 2011). 
Far more research has been conducted on the individual elements; i.e. chitosan and calcium carbonate (but not chitin and aragonite). Chitosan has been extensively investigated in medical use because of its biocompatibility and healing properties (Park \& Kim, 2010). Chitosan improves the rate of bone healing via the acceleration of blood vessels formation, which is essential for the nutrient intensive bone growth process, but other mechanisms are also involved (Mathews, Gupta, Bhonde, \& Totey, 2011). Whether chitin behaves in a similar manner is less known but may be less effective since it tends to be hydrophobic instead of hydrophilic (cells prefer hydrophilic) (Chang \& Wang, 2011) and activates different responses in vivo (Bueter et al., 2011). Interestingly the use of chitin as a structural polymer has not extensive possibly due to the difficulty in manipulating it and recreating the strong designs in nature (Fernandez \& Ingber, 2013). A key question remains whether the synthetic nacre has truly mimicked the microstructure of the natural form, replicating the high strength characteristics. The likelihood of disparate properties is high - for instance the degree of mineralisation is currently significantly different: less than $40 \%$ versus $95 \%$ in the natural form.

Calcium carbonate in the form of aragonite has been used as commercial bone substitute, in the form of coral which is used for its interconnected porous structure similar to cancellous bone (Demers et al., 2002), but appears less researched in medical literature than hydroxyapatite, a form of calcium phosphate, which is popular due to having a similar crystal structure and chemistry to bone. An advantage of synthetic nacre may be the aragonite, which is stronger than the calcite, a more easily synthesised calcium carbonate crystal structure, but more soluble (Roberts, 2009), without organic contaminants. The aragonite in itself, in addition to nacre previously mentioned, can also support bone growth (Shafiu Kamba \& Zakaria, 2014).

As part of an enterprise development the commercial advantage over other materials, particularly hydroxyapatite and the synthetic polymer polylactic acid (PLA), was investigated. In medicine when a new product arrives that is an equally, or more, expensive material such as synthetic nacre there must be a protectable significantly improvement in patient outcomes for it to be adopted. For chitin there is the possibility of improved rates of healing and bone mineralisation. As a natural polymer it also degrades into non-acidic by-products (Azevedo \& Reis, 2005), unlike PLA, and may regulate the immune system (Li et al., 2013). In aragonite it is less clear whether there is a better outcome but a potential argument is that hydroxyapatite, surprisingly, may not be completely absorbed in vivo (Ranjan, 2011). The multiphasic interface that may form between synthetic nacre and natural bone could also be an improvement as a major issue in orthopaedic implants is maintaining fixation (where the implants become loose and allow slippage or movement). 


\section{Porous structure requirements for osteoconduction}

For a biomaterial to have high quality osteoconductive properties it needs a porous structure that meets certain requirements for pore sizes and interconnectivity. For bone cells there is an ideal macro pore size of 200 to $300 \mu \mathrm{m}$; though there is disagreement within the literature (Zhou, Ma, Li, $\&$ Yao, 2011). Bone growth is a nutrient intensive process that relies on neovascularisation, the development of new blood vessels in abnormal (e.g. substitute bone) tissue ("neovascularization," 2003), throughout the biomaterial scaffold to supply oxygen - the limit of diffusion of oxygen in vivo is $100 \mu \mathrm{m}$ (Rouwkema, Rivron, \& van Blitterswijk, 2008). This means that isolated pores are not sufficient as they need to be open or interconnected with each other. The degree of interconnectivity should be very high (100\%) and support the shortest path routes through the implant biomaterial as blood vessels form very slowly (Rouwkema et al., 2008). The diameter of connecting tunnels between pores is ideal, for osteoconduction, when $50 \mu \mathrm{m}$ (Lu et al., 1999); given the size of osteoblast cells is 20 to $30 \mu \mathrm{m}$ this appears reasonable. However the ideal size for blood vessels is closer to $5 \mu \mathrm{m}$ (Whang et al., 1999) revealing the importance of multiple level porosity, micro as well as macro.

\section{Dependency analysis}

Dependency analysis is a tool to examine relationships within a system. For example root cause analysis is a common method used in engineering to find the original fault that caused a failure. Other variations include feature dependency, used to identify common features in multiple products (to optimise manufacturing) (Lee \& Kang, 2004), and functional dependency, used to examine how changing an item will affect the rest of the system (Garvey \& Pinto, 2009).

For the bone project an informal dependency map (Figure 5) revealed that the synthetic nacre, in its current status, has little protectable or essential features linked to osteoconduction; in particular it shows that osteoconduction is almost solely dependent on the macro and micro porous structure and not the type of material (as long as it is not toxic and supports cell growth). Furthermore while chitosan/chitin is known to have healing properties it is not specific to synthetic nacre; any other material, such as PLA, can incorporate it nullifying the advantage in the bone project enterprise development. 


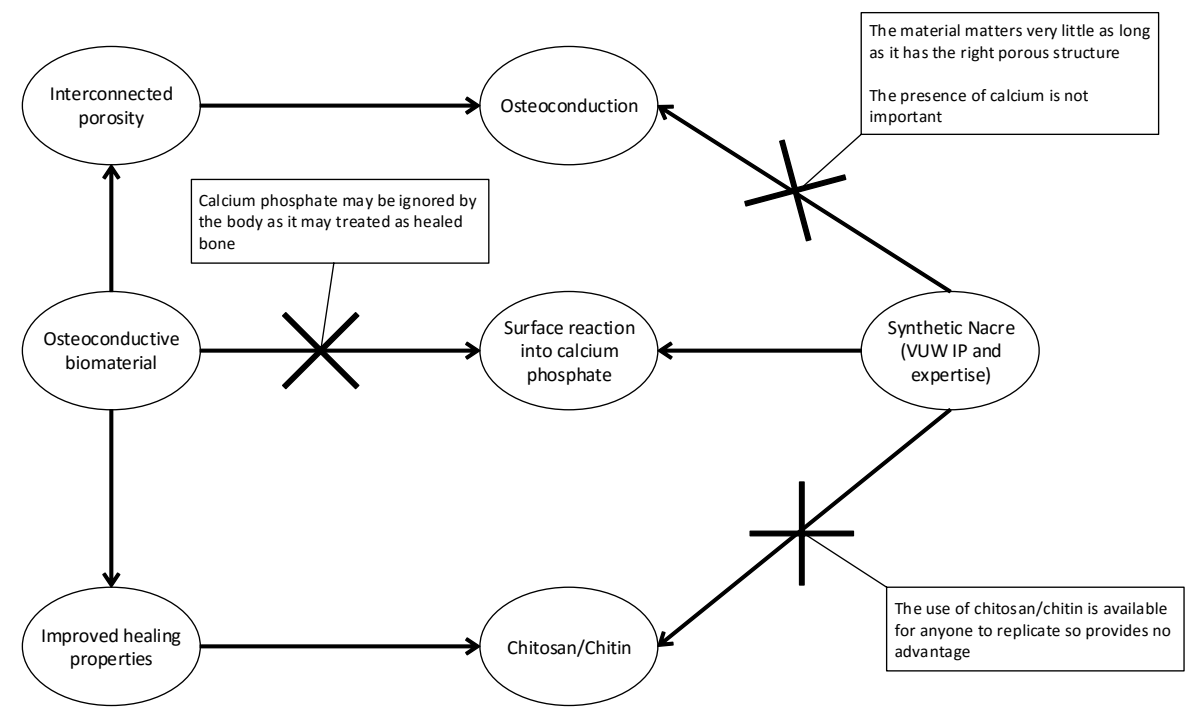

Figure 5 Dependency analysis between synthetic nacre and osteoconduction

By examining osteoconduction alone (Figure 6) this assessment drove the bone project's decision to prioritise the incorporation of the porous structure, alongside the VUW research group's diffusion and distortion issues regarding manipulating the chitosan/chitin scaffold, and strength testing before the biological in vitro studies to see whether the synthetic nacre enabled osteoconduction to occur.

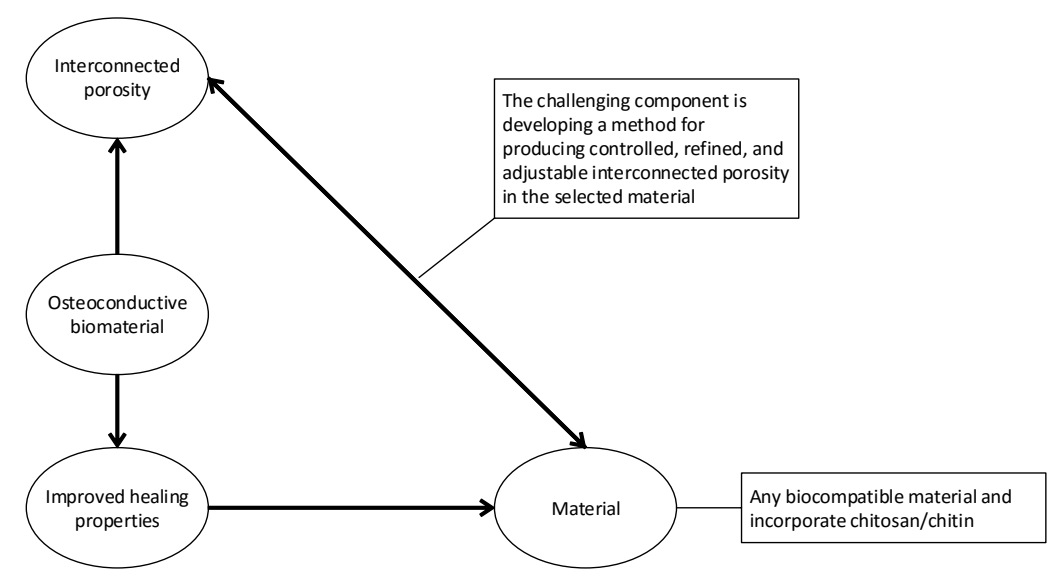

Figure 6 Dependency analysis for osteoconduction

It naively argues that features synthetic nacre has that might be considered useful; the presence of calcium, chitosan/chitin, calcium phosphate reaction; are not actually necessary to achieve and osteoconductive biomaterial. All it needs is to allow cells to proliferate and have an ideal macrostructure for vascularisation. This is could be an overly simplified explanation however nonbiodegradable orthopaedic implants made of porous titanium have been investigated for bone ingrowth (Hirschhorn, McBeath, \& Dustoor, 1971; Lopez-Heredia et al., 2008). 
Dependency analysis, in previous experience used as a tool to find defects and original causes of failure, has been used in the development of the product specifications for the bone project's synthetic nacre biomaterial. By transferring across a skill from engineering and applying it to tissue engineering design the most important product features were identified and provided the team with a recommendation for the material development priorities.

\section{Porosity and strength}

The market research found by the team identified that while substitute bone biomaterials in the form of void fillers were widely available there was a lack of load bearing biomaterials with good osteoconduction. Achieving a high strength in combination with the desired porous structure became a target capability for the synthetic nacre material (an initial push into this direction was the advantage of aragonite having a higher strength than the more common calcite (Roberts, 2009)).

The method developed by the VUW research group to fabricate synthetic nacre mimics the selfassembly of the natural form. The natural form of the material is relatively similar, but with a lower maximum strength in compression (it is also believed that the synthetic version does not approach the maximum in its current state), to compact bone (Wegst \& Ashby, 2004). Unfortunately there is an inverse relationship between porosity and strength which effects osteoconductive biomaterials (Karageorgiou \& Kaplan, 2005). This was used to predict, once the desired porous structure is incorporated, that the final strength of the future biomaterial will have a high risk of not meeting the target for strength in compression of $230 \mathrm{MPa}$. It was felt that without at least the possibility of increasing the material's mechanical strength the project should have been abandoned.

Two pathways forward were provided, in addition to the possibility of using self-reinforcing techniques (p. 7), to supporting continuing the project:

a) The VUW research group had previously incorporated magnesium into the material, which along with the higher percentage of chitin content than natural nacre, and it was proposed that as much as $30 \%$ magnesium could be included in the mineralisation process. It may be that altering the mineralisation could improve the material's strength.

b) A specialised structural macro porous design to improve the strength; at its basic level essentially a sparse network of pores that allow osteoconduction but retains more material and therefore strength. This would require a non-traditional manufacturing process such as 3D printing. Traditional techniques such as foaming, gas bubbling, use of porogens, 
freeze drying, are generally not able to produce this type of structure without losing strength characteristics.

The precise control over the interconnected macro porous structure design needed to achieve a biomaterial that has both high quality osteoconduction and mechanical strength resulted in the decision that not only was 3D printing an option, as developed by the VUW research group, but it, or a similar high resolution additive process, would be required.

\section{Degradation}

Biodegradable is the property of the implant biomaterial breaking down or being absorbed in vivo over time. Controlling the rate of this degradation is a key requirement for any biomaterial since it needs to match the rate of healing (Raghunath, Rollo, Sales, Butler, \& Seifalian, 2007). If the implant degrades too fast the bone will not have healed in time; if it is too slow the healing process will be impeded. Importantly however is the biomaterial must be $100 \%$ biodegradable; PLA for instance can leave crystalline remnants of material within the bone long after complete healing (Bergsma, de Bruijn, Rozema, Bos, \& Boering, 1995); not unlike traditional implants made of metal and negating some of the benefits of being biodegradable.

As with porosity and strength there is an interdependence between porosity and degradation (and between degradation and strength since additionally the mechanical properties of the implant biomaterial will change over time as it is degraded). Ideally the rate of degradation and the strength of the biomaterial would be decoupled to enable each to be individual controlled (Yildirimer \& Seifalian, 2014). For the synthetic nacre material this would involve altering the chemical structure and mineralisation and it was felt true decoupling is unlikely but partial decoupling would still be useful.

\section{Summary}

This review and assessment helped provide the final current status of the synthetic nacre material in relation to the market application the bone project team was targeting. It revealed that there was no reason synthetic nacre could not be a good biomaterial, for both osteoconduction and strength, but that the essential porous structure required is missing. The potential healing properties of chitin, while advantageous, are not as important as a developing a precise method to fabricate a highly interconnected porous structure with high strength (the same as cortical bone).

For the bone project's market niche of biodegradable load bearing osteoconductive implant biomaterials this meant the technical targets for synthetic nacre require balancing the porous structure, mechanical strength, and degradation characteristics. The osteoconductive properties 
rely on both an ideal porous structure and even strength (since bone remodelling is adaptive and bone healing differently depending on the forces applied (Sikavitsas, Temenoff, \& Mikos, 2001). The biomaterial's strength is inversely related to porosity, but needs to meet certain targets to be used for load bearing applications. The degradation characteristics need to be precisely controlled and will rely on the chemical and porous structure of the synthetic nacre material.

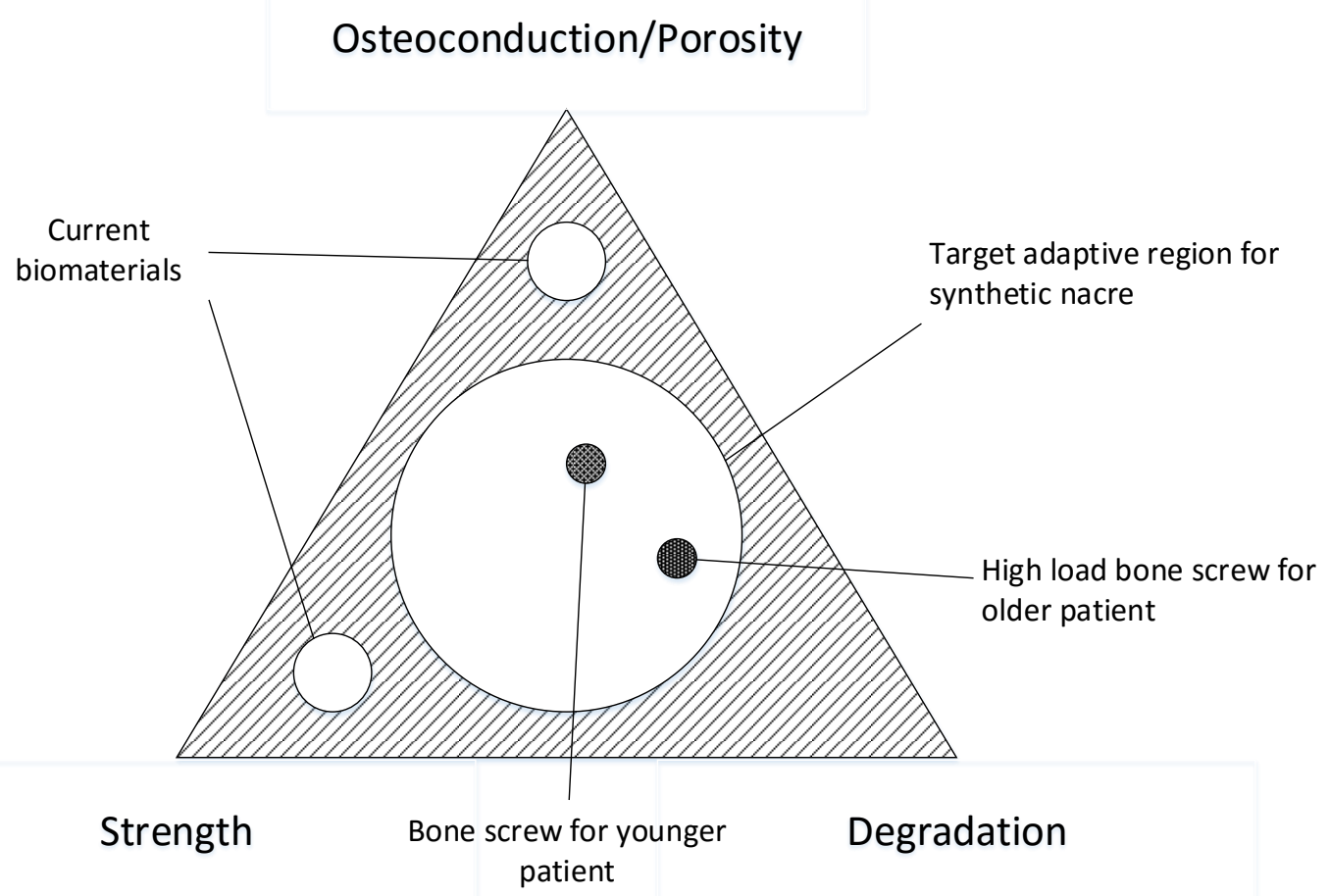

Figure 7 Conceptual relationship between porosity, strength, and degradation characteristics. Actual relationships will are unknown but likely non-linear. Shows potential optimal regions for different materials and products.

The bone project business model relies on synthetic nacre becoming a platform biomaterial for multiple implant types and applications or multiple product lines. Many of these products will need a different compromise and optimisation of the above interrelationships (Figure 7).

It is believed the 3D printing method, already conceptual shown to work by the VUW research group, is more suitable than traditional techniques to achieve the precise control over the porous structure; indeed traditional techniques may not be able to fabricate it without compromising the strength of the biomaterial. 


\section{CHAPTER 4 SINGLE MATERIAL AND MANUFACTURING SYSTEM}

\section{Introduction}

Towards the end of the yearlong course, and as the understanding of the market progressed, the initial concepts around what the product was and how a business would need to be structured to succeed evolved into more advanced concepts. The porous structure and other features required, and the obstacles with the current state of the synthetic nacre fabrication process, were now known. The concepts introduced here, and described in the business case, are in preliminary planning awaiting the material development of the desired features within the synthetic nacre. This section primarily reflects on how mechatronics and engineering concepts influenced the enterprise development towards the single material and manufacturing system.

\section{Single material and manufacturing system concept}

The single material and manufacturing system design concept aims at providing a better solution to two current approaches to manufacturing biomaterial implants for orthopaedic surgery.

Firstly, current biomaterial orthopaedic implants, such as a biodegradable screws, use a customised and increasingly complex, traditional, top down fabrication approach as oppose to a simplified, bottom up, additive manufacturing, method such as 3D printing. Each implant and biomaterial type goes through a separate manufacturing process. Secondly, each biomaterial, with a set degradation, porosity, and strength, is limited to a few applications (strictly speaking there is some customisation in a variety of commercial products, particularly in PLA, but not to the extent that the bone project is aiming to achieve).

By combining 3D printing and a material suitable, given adequate ranges for its porous and chemical properties, for a wide range of applications the single material and manufacturing system can provide tailored material properties for a broad range of patient and implant types. This approach is attractive from an enterprise development point of view because the manufacturing process only has to be designed and developed once; new implant designs can be manufactured without starting from scratch to design a specialised fabrication method or process. Additionally due to the likelihood that synthetic nacre will need to proceed into the more expensive regulatory pathway of clinical trials no single implant design or type will provide a return on investment - the synthetic nacre biomaterial will need to support for multiple applications. 


\section{Control systems}

Control systems theory was the starting point that contributed towards changing the direction of the bone project enterprise concept towards the single material and manufacturing system. The interdependence and balancing act between porosity, strength, degradation, was viewed as a system which needed to be controlled.

A system is a collection of objects in an ordered form that can be described by the cause and effect relationships between its inputs and outputs (Mahmoud \& Xia, 2012). A system can be anything but for the case of the synthetic nacre biomaterial it can be described as the chemical nature, porous structure design, and other properties and components that are combined with the human body and its biological bone growth and degradation processes. The inputs include the porous structure design while the outputs include the capability for osteoconduction in vivo and biomaterial strength.

A controller can be viewed as an additional component that automatically adjusts the inputs to obtain either set or optimal outputs. For biomaterials not all inputs can be adjusted, such as the processes within the human body, but the design of the biomaterial and its chemical nature, within constraints, can be. For the development of the bone project enterprise product concept the key point is that, in theory, a control system could be used to automatically achieve the best osteoconduction possible, by adjusting the porous structure, given set material strength targets needed for different orthopaedic implant applications.

\section{Adaptive manufacturing}

The single material and manufacturing system concept described above is a form of an extremely flexible production process called adaptive manufacturing. Adaptive manufacturing is a mass production manufacturing system that responds, by reconfiguring or adjusting itself, very quickly to changes in volumes, products, and customer requirements. A very simple example of adaptive manufacturing is when individuals can order their car with a specific colour which is then directly included in the assembly line without human intervention - the ordering system selects which colour each car will be painted, based on customer input, as it goes through the factory. More formally adaptive manufacturing can be described as "continuous automatic adaptation of manufacturing resources and production processes to their evolving environment" (Rolstadås, Henriksen, \& O’Sullivan, 2012). 


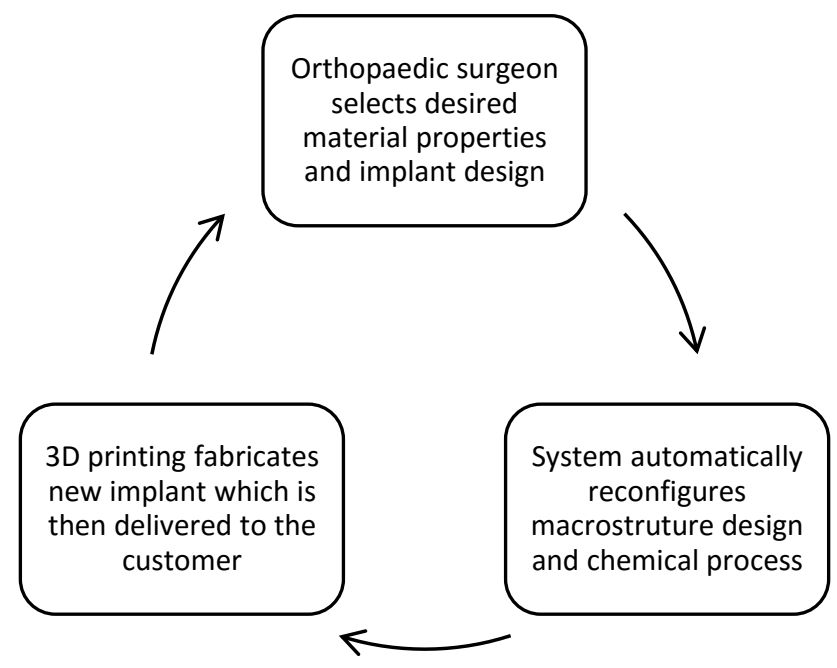

Figure 8 Adaptive manufacturing feedback system

An important component of an adaptive manufacturing system is real time feedback (Figure 8) (Rolstadås et al., 2012); the information source for knowledge it uses to base changes upon. In the case of the bone project this would be the input from orthopaedic surgeons as they select the shape and specific values of the different properties (i.e. strength, porosity, and degradation rate). A, for example, 3D printer could then produce the synthetic nacre product to their specifications without an implant designer's specialised intervention.

\section{D printing and control systems}

A combination of two preceding developments during the bone project the made the transition to an adaptive manufacturing system, despite being far more complex, a more gradual conceptual step change to the direction of the project: the belief that 3D printing is required, rather than optional, and the approach to viewing the balancing act between the ideal biomaterial properties as a control system.

$3 \mathrm{~d}$ printing has recently become popularised with machines such as the Makerbot which can take a design as a computer file and build it in plastic layer by layer over several hours. Stronger materials, such as metals and different high grade plastics, (or methods that can use them) have been developed which are not restricted to prototyping but can and are being used for the final product. Such a slow additive process is now being tested at scales approaching factory production levels (though it will may never be suitable for mass production in millions of units) (The Economist, 2013). The VUW research group had already developed a 3D printer that conceptually proved it could work as an optional fabrication method. As the need for precise control over the sparse 
interconnected pore network structure in the specifications was developed it was believed traditional techniques would not work and the 3D printer would likely be the only method suitable.

If a mathematical or statistical model of the synthetic nacre biomaterial, incorporating a range of porous structures, strength, and degradation properties, could be developed a control system could be implemented to adjust the compromise and optimisation between the three properties. This could allow implant designers, or surgeons in an adaptive manufacturing system, to select their desired strength and be able to view the best design case for osteoconduction (porous structure) and degradation characteristics. Finally a control system is often a feedback system which, if (Figure 8) and (Figure 9) are compared parallels an adaptive manufacturing system.

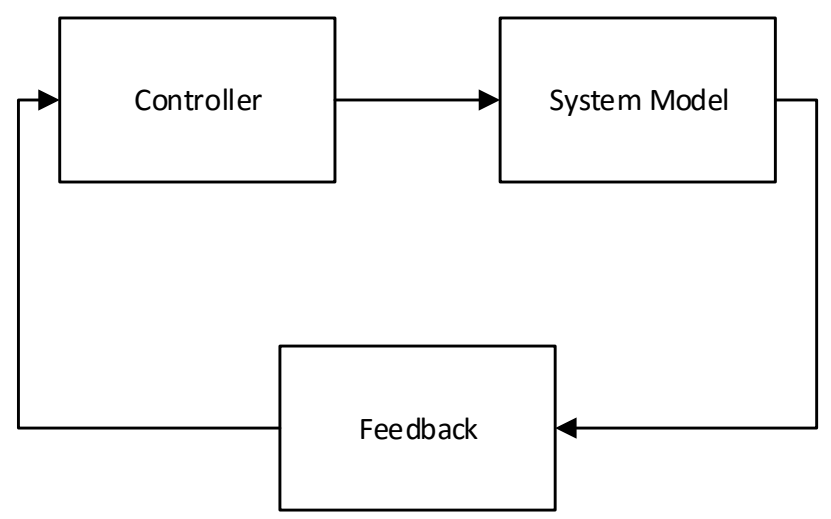

Figure 9 Basic control system overview

Combining the two, 3D printing with a control system, and acknowledging the branch of research into automatic design algorithms for bone like microstructures, made the step and conceptual progression towards adaptive manufacturing, in relation to the bone project, relatively simple - all the necessary precursors had already been developed. The single material and manufacturing system concept became the solution enabling synthetic nacre to be developed into a biomaterial platform for multiple products.

\section{Personalised Medicine}

Adaptive manufacturing with real time input from customers is a platform that could help enable personalised medicine in the medical implant arena. Personalised medicine, most commonly associated with drugs, is tailored and customised therapy or treatment specific to an individual or subset of (with the premise of supporting better outcomes) (Brunicardi et al., 2011). In the case of 
the bone project the synthetic nacre degradation rate could be, for example, matched against the rate of bone growth (extrapolated from genetics, gender, age, race, and diet) within the patient. The concept combining an additive fabrication process with a control system interfaced directly with customers learnt during the bone project enterprise development experience could also be useful for other applications in the future: the mass production of customised products is a growing trend and not limited to personalised medicine (Sherman, 2013).

\section{Toyota Product Development System (Lean Product Development)}

There was a third influence, or rather parallel, that helped direct the concept during the bone project - this was not intentionally implemented but forced because there was no exact specific target application, only the generalised target of a load bearing bone screw. The control system balances the different relationships between the properties to reach an optimal point given certain requirements; these would likely be in the form on non-linear graph curves. If it was not for the specification developed, for a high strength biomaterial with porosity enabled osteoconduction, these curves or profiles (to be built as the synthetic nacre material is developed) would be the information used to design new implants.

This is a parallel to elements of the Toyota Product Development System - not to be confused with their more famous Toyota Production System - or lean product development (LPD). In this situation no specifications are developed but trade-off curves; for example between cost, performance, size, weight, etc.; for the different subsystem properties are built which are then used to select the optimal component (Sobek, 1997, p. 219). The whole concept is based around creating a steady flow of new products (Kennedy, 2004) where interchangeable subsystems can be reused or developed independently (for the most part as interfaces are still kept flexible). For example if the new air conditioning unit is not performing as expected the previous, tested and working, design can be used without requiring the rest of the system to be reconfigured, delaying the final design, and release of the new car model. The issue and difference for the bone project is that the "interchangeable" features are highly interrelated and not interchangeable; requiring a control system to reconfigure the whole system automatically.

In the bone project setting up the single material and manufacturing system was secondly conceptualised to implement the biomaterial platform for a flow of new products; in the first instance it was developed to make the enterprise development more robust to unknown changes ahead. Despite narrowing the target market application for synthetic nacre to bone screws there was no specific implant specifications developed (the target profiles detailed in the appendix are for a general load bearing biomaterial with good osteoconduction, it is not specific to any individual 
implant type and application). This forced the bone project direction towards developing the single material and manufacturing system concept, and creating a partial analogue to the Toyota Product Development System, to enable the material to incorporate any changes once the market expectations and demands were known.

\section{Summary}

Reflecting on the steps taken during the design process has revealed an incremental evolution leading towards the single material and adaptive manufacturing concept. The requirement for 3D printing over traditional manufacturing techniques and viewing the compromise/optimisation between porosity, strength, and degradation as a system that needs to be controlled made the step and increased complexity to an adaptive manufacturing system concept a natural progression.

The lack of exact market niche and knowledge of customer requirements for that forced a flexible approach to developing specifications that is analogue to components of the Toyota Product Development System. Having unwittingly approached the bone project using this approach, where the central idea is to produce a flow of new products, the groundwork for the bone project's biomaterial product platform concept had been completed; awaiting the manufacturing concept progression contribution. 


\section{CHAPTER 5 DESIGN PROCESS REFLECTION}

\section{Introduction}

The bone project team went through an unstructured process towards the commercialisation of the synthetic nacre material with the guidance of the business mentors. The practical experience was used as a learning experience by reflecting on how and why decisions, events, and understanding occurred as they did. The previous chapters can be divided into steps using the role of engineers to solve problems. Here the basic stages of problem solving (Figure 10) are mapped onto the engineering design process and discussed.

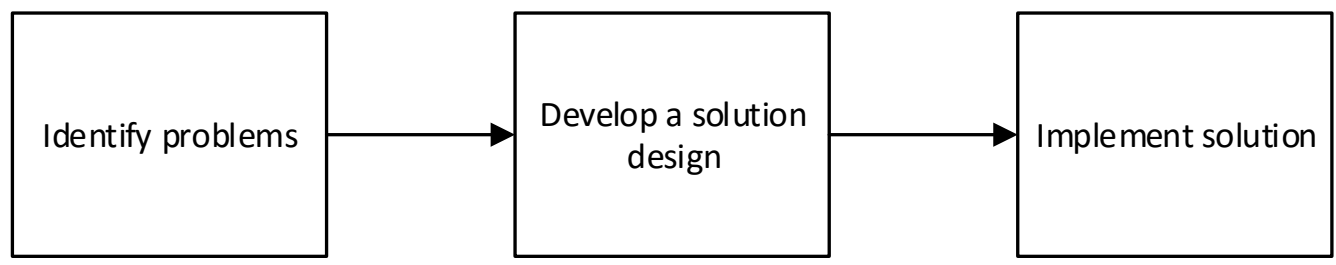

Figure 10. Bone project solving problems stages

\section{Engineering design process}

A simple example of a common and successful engineering design process is shown in (Figure 11). The bone project deviated slightly from this from the beginning since the problem was developed in-house in a technology push situation, where a market application for a technology is sought rather than a client requesting a solution to a problem. Instead the original objectives chosen by the team were based on what was believed to be the most likely application for the synthetic nacre material: as a biodegradable load bearing osteoconductive biomaterial for orthopaedic implants. Whether this was a true problem or not would wait upon the steps towards market validation later in the project.

From this starting point the requirements for osteoconduction were developed. Finding the necessary features (functions) and limitations (constraints) affecting biomaterials and synthetic nacre such as the lack of porous microstructure and limits of diffusion. The design concept for a sparse pore network and single material and manufacturing system were proposed. The bone project never reached a point where the solution could be implemented and tested. It is relatively 
simple to see that the basic problem solving process (Figure 10) maps onto the initial stages of the design process (Figure 11).

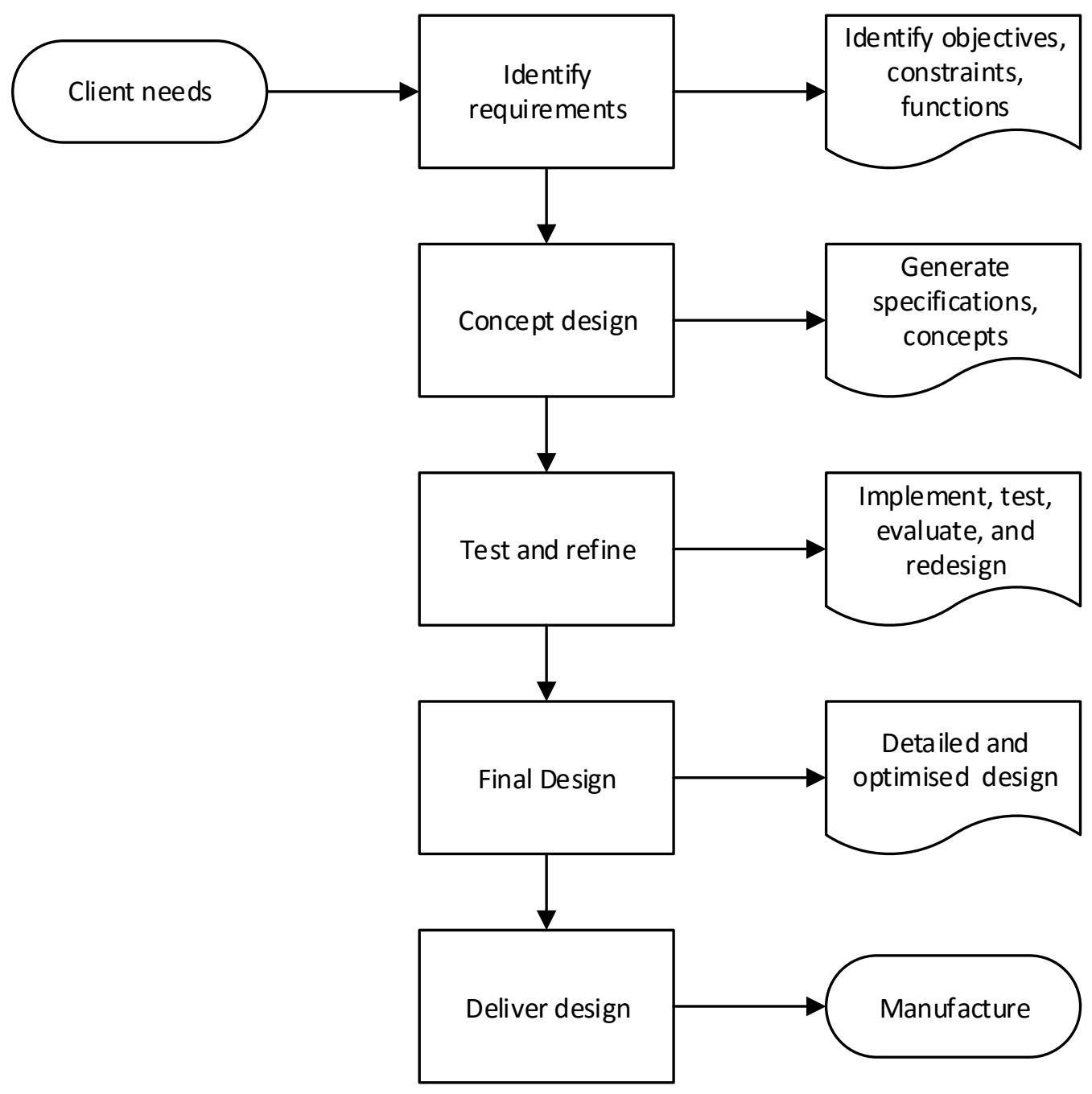

Figure 11. An engineering design process; adapted from Avramenko (2008)

Any design process should test and evaluate the success of the design, this is an iterative process which at is very basic level follows (Figure 12). The bone project did not complete a single complete cycle; the concept for the sparse pore network or even the whether the concept has met the client needs has not been tested. The only task that may be considered part of the testing stage was the degradation experiment in phosphate buffer solution however this is not testing the manufacturing and biomaterial design concepts developed (these had not been generated at that stage). In summary no testing or validation of the final design ever took place. 


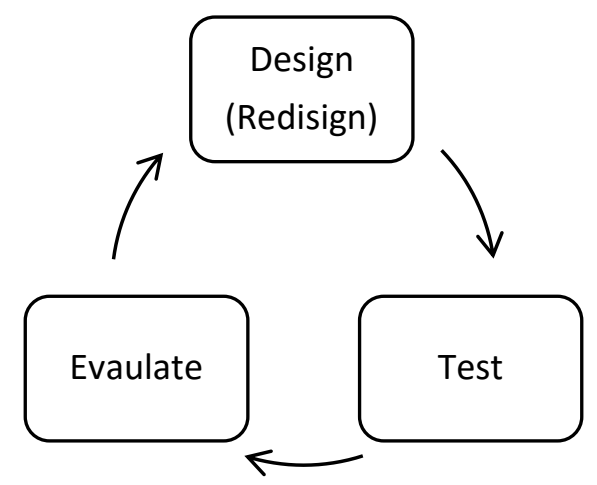

Figure 12 Iterative design cycle

Part of the lean philosophy for start-ups is to fail quickly and an objective of the MATE programme is to allow a safe environment for failure; that the bone project team was not prepared to fail the project during the course of the year again suggests that the objectives were not met - even if the design concept becomes successful the project did not proceed fast enough for it to be evaluated. If the synthetic nacre and single material and manufacturing system concept is a success in the future this project could still be considered a failure in that the design, test, evaluate loop was never completed during the course.

Additionally, from one viewpoint, the adaptive design taken can be argued as an attempt to make the generalised untested specifications (due to not being known for specific implant applications) more robust by requiring the ability for individual properties of the synthetic nacre material to be flexible and changeable. This could allow the best configuration for an implant to be found (by creating a system that gives surgeons the power to tailor the synthetic nacre material) after the technical and enterprise development is closer to completion - essentially shifting the deadline for validating the biomaterial capabilities/specifications by adding flexible features and complexity to the whole design concept. The bone project enterprise and design concept development were artificially extended to cope with ambiguity: the lack of questioning and validation of a specific target market niche and its requirements.

\section{Concurrent Engineering}

The above discussion is perhaps overly critical considering the complex and multidisciplinary nature of tissue engineering and medical product development; a more productive view can start with examining the chosen manufacturing method of 3D printing. 3D printers are often restricted to a single or set of, often propriety, materials which were developed in conjunction with the specific additive manufacturing process used. An example of this is the lithography used in the original 3D printers require requires a specific (toxic) type of polymer cured by UV light. 
Concurrent engineering is a process where all components required to bring a product to market and beyond: the product design, manufacturing process, marketing strategy, maintenance, and more; i.e. the whole product life-cycle is all developed in parallel and allowed to influence each other (Jarvis, 1999). This is in contrast to the waterfall methods where first the product is design, then tested, then the manufacturing process developed, and so forth. It has a disadvantage of being more complex and difficult to implement (currently it is used primarily by aerospace and aviation companies such as at ESA's Concurrent Design Facility (ESA, 2012)) but reduces the time to market significantly.

A key feature and requirement of concurrent engineering is an extended preliminary design and planning process. This is exactly what the bone project has completed, a preliminary, untested, but extensively developed design concept developed over the yearlong course - suggesting it may be ideally set up for using concurrent engineering in the future.

\section{Summary}

The design process followed within the bone project team regarding the development of the single material and manufacturing system concept has been discussed in relation to the role of engineer to solve problems and in terms of its failure to complete the design process or test/validation stages. The extended preliminary design stage supports the future possibility of using concurrent engineering concepts to development the synthetic nacre material and 3D printer machine. 


\section{CHAPTER 6 SECONDARY COMMUNICATION ROLE}

\section{Introduction}

A goal of the MATE programme and the bone project was to examine the role of an engineer within the enterprise start-up. The initial hypothesis was that the role of any engineer within a team is to solve problems, which has been partially demonstrated during the bone project, however upon reflection a secondary role in communication became apparent. Adding to the importance of communicating, from in the laboratory with the VUW research group, is the influence of tender specification writing in the proof of concept and illustrations in the bone project presentations.

\section{Proof of concept}

As part of establishing a business case and data package for using synthetic nacre as an orthopaedic implant biomaterial a proof of concept section describing the individual tests and passing values required to proceed to the next stage in product development; the very expensive in vivo studies in animals. This makes use of many of the tests that would be required during the technical development of the material but formatted in a go/no-go stage gate form.

The responsibility for the mechanical and degradation components were included in the technical role while the biological aspects were undertaken by Christina Houlihan. This involves medical regulations and the role of standard testing procedures (for example ASTM standards). For enzymatic hydrolysis degradation of chitosan/chitin there is no standard in vitro testing procedure; only for non-enzymatic hydrolysis by which polylactic acid and other synthetic polymers are degraded. Therefore a degradation test had to be constructed from suggested procedures, primarily developed from (Azevedo \& Reis, 2005).

An approach similar to writing tender document specifications for engineering projects was applied to the bone project's proof of concept testing procedure. The central concept being that each and every specification in the contract should have an accompanying test procedure. Each test procedure should be able to be completed within a reasonable time and cost and have a specific pass or fail value that avoids ambiguous, and challengeable, results. In the case of the bone project this was not always possible as - specifically referring to the rate of debris released from the degrading material in vitro - there is not always a pass or fail value (for in vivo this would be measured by the presence of adverse reactions). In these cases subjective opinions will still be relied upon.

Assuring each specification had a matching test clarified what was required at each material development stage. The order of testing was prioritised, with input from the dependency analysis 
(p. 11), based off critical pathway (osteoconduction and strength testing is not possible until the porous macrostructure is incorporated), cost, and risk (delaying expensive and low risk tests). For instance the inverse relationship between porosity and strength suggests the low cost compression testing, the first test recommended after $\mu$-CT scanning the macrostructure, has a high chance of failure.

As this part of the individual role within the team did not identify or solve any problem in the bone project enterprise development it can be separated into a secondary role of communication. The proof of concept document is there to help and convince others of the technical progress of the material development.

\section{Presentations}

A number of presentations are a compulsory component of the MATE course and illustrations, in the form of storyboard comics, was used as a visual aid to display information and concepts in a non-technical and humorous manner. The original presentations in the beginning of the year were very number orientated and used dense bullet points to describe the complex porous structure required to achieve good osteoconduction. Even to the business mentors who were familiar with the bone project found the explanation confusing and suggested a more visual approach may help. It was from this initial suggestion that the final presentation developed with a sample below for reference.

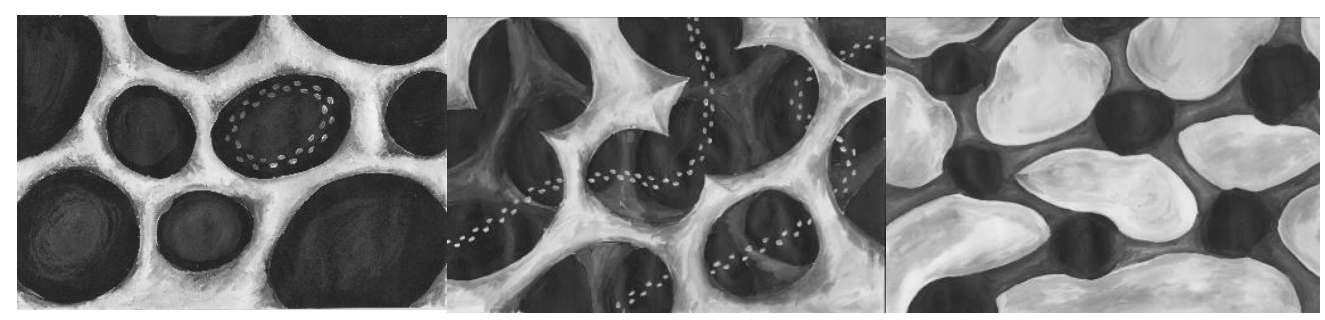

Figure 13 Artistic impressions of the different porous structures: isolated, interconnected, and sparse pores.

As well as providing clarity to the explanation this also helped solve a number of conflicts with the differing terminology used in the different medical, scientific, and engineering fields: conductive, open, connected, interconnected pores appear the same and helped communicate the sparse pore network to the audience. In addition this help explain to the rest of the project team why traditional techniques for fabrication would not work and that 3D printing would, most likely, be required. In the future diagrams representing similar technical descriptions, instead of the less form illustrations, could provide a more professional appearance. 


\section{Summary}

A secondary role to engineering; that of communication; was demonstrated during bone project enterprise development through working with the VUW research group, document creation (proof of concept plan), and presentations. Illustrations were used to communicate the technical aspects of the biomaterial design concept to the rest of the team and outside audiences in an understandable fashion. This helped guide the direction of the project since it made the unintuitive and complicated interdependence between and requirements of the three properties: porosity, strength, and degradation; comprehensible to the team and influenced their decisions. 


\section{CHAPTER 7 COURSE STRUCTURE AND TEAM DYNAMICS}

\section{Introduction}

The team environment and course structure have an important effect on the practical enterprise development component and individual role. The following section attempts to include relevant aspects of the course structure, other bone project team members' work, and how it affected the progression and direction of the bone project in relation to the engineering design concept and process.

\section{MATE project selection and business model canvas}

The business model canvas (Osterwalder, 2010) was the tool used to help guide the participating students through screening the research provided by the project champions. In discussions with the business mentors it was suggested that this had the effect of encouraging the formation of a business around the synthetic nacre rather than investigating other ways of commercialisation the material such as licensing or developing a relationship with a partner. This may have biased the team towards the very complex single material and manufacturing system concept, which is able to support a standalone business, instead of a simpler approach with lower barriers to entry. If another method had been used to screen the projects and develop the initial business concepts it raises the question whether the team would have arrived at the same end point in the project.

On a positive note Steve Blank's insistence of leaving the building and talking to customers (Blank \& Dorf, 2012) is reflected, to an extent, in the project with Michael Mettrick's interviews with orthopaedic surgeons completely changing the direction of the technical design by raising the issue of the compromise between high strength and good osteoconduction. This, along with reliable precise control over degradation, fed into the tailored material properties (porosity, strength, and degradation) concept.

A philosophy of lean start-ups being to fail quickly and fail often (Blank \& Dorf, 2012) (rather than expending effort on developing a business or product without testing whether anyone will pay for it). This is done by following an iterative process to validate decisions with the real customers using a mock up or a minimal viable product. A number of invited speakers also spoke about the concept of selling an imaginary product, assuming the synthetic nacre can and will be developed to meet the desired specifications, was strongly suggested to find out if people wanted it (and how they wanted it) before building it (though not having a refined product concept or knowing how the bone project business case would appear until near the end this was perhaps premature). That the bone project enterprise never followed this approach reinforces the argument that the extended 
design process, without testing, in the bone project lead to the enterprise development being unsuccessful.

\section{Safe failure environment}

Failure is part of business and start-ups and within the MATE programme an objective was to provide a safe environment for failure allowing new ideas to start or to pivot around issues blocking progress towards a successful enterprise. As the bone project had been dropped by a team in the previous year due to an inability to progress it was a known risk it could happen again. The small number of initial projects (in the current year) necessitated creating another, revolving around using eye tracking technology in education, to provide a fall back option. This went as far as interviewing an IT coordinator at a local school who provided a detailed commentary on the issues they were facing. No team with a singular vision formed around the project and the magnitude of the limited budget within schools was now known leading to the projects demise.

This meant there was no suitable projects for myself, or other team members, to take on in the event the bone project stalled or failed. During the course of the year this did, to a degree, happen at some points but ways were fashioned to get around it (though this could be more positively referred to pivots around blocking problems). When the current development status of the synthetic nacre material - particularly around the limited wall thickness - and inverse relationship between porosity and strength were known there was an opportunity for the team to decide upon either dropping the research or finding an alternative application for it.

Other applications were ruled out - due in part to the original justification for targeting a medical application being the very high cost (\$2.1 per gram) of the raw materials needed to manufacture synthetic nacre. That the material was still under active research however allowed the team to keep progressing with the project on the basis that the synthetic nacre could be developed to incorporate new features or meet more strenuous requirements. By avoiding ending the project then (by returning it to the VUW research group with suggestions on how to proceed) two things occur: 1) the philosophy of failing quickly was not followed and 2) time was provided that allowed the single material and manufacturing system concept to be developed (without testing whether the material concept meets the requirements or is desired by orthopaedic surgeons).

\section{Role evolution}

At the start of the year the current status of the synthetic nacre material and the method to produce it were thought of as fixed and for the most part fully developed. The 3D printer developed by the VUW research group was thought to be more robust and refined then it turned out to be. 
The thickness limitation of the material was believed to be in the 2 to $5 \mathrm{~mm}$ scale (not the less than 0.5 to $1 \mathrm{~mm}$ after mineralisation it actually was). From this optimistic viewpoint of the synthetic nacre material the personal role within the team, and the initial milestones of the project were developed and decided.

As the previous year's team, who had also completed a small component of work on the synthetic nacre material, had stalled due to not being able, or willing, to work with the material without the research team's help it was decided to learn the chemical process used to manufacture it. This would have had a three-fold task: 1) transfer knowledge across from the VUW research group allowing the bone project team to work independently 2) provide a means to fabricate specimens for proof of concept testing and 3) help me identify whether the chemical process would scale up.

In conjunction, as it took some time before safety training was completed, the medical literature around bone biomaterials and tissue engineering was reviewed. In particular the specialised porous structure required for osteoconduction lead to absorbing many of the biological aspects initially assigned to Christina Houlihan (whose role also evolved to other areas).

During the time in the laboratory learning the process the actual development status of the synthetic nacre material was uncovered. The chemical process was not fixed and could be altered to achieve different ends. The 3D printer developed by the VUW research group had tested the concept but was unrefined and unreliable. The limitation on thickness was reduced to less than $0.5 \mathrm{~mm}$. This required an adjustment to the role and the objectives for the enterprise development project.

The role within the team changed from a manufacturing to a product development - in particularly applying the multidisciplinary nature of mechatronics to the equally, but differently, multidisciplinary nature of bone tissue engineering. The primary questions to answer were: 1 ) what are the characteristics of an ideal biomaterial (in the biodegradable osteoconductive load bearing niche), did synthetic nacre have these, and if not, could they be incorporated in the future? 2) Given the very high raw material cost of \$2.1 per gram how can a significant advantage (the proposed solution being good osteoconduction and high strength within the same biodegradable biomaterial) be engineered into the synthetic nacre material over competing products?

\section{Team dynamics and building}

The team, and specifically the work completed from other roles, had a beneficial effect on the individual role and the development of the biomaterial specifications concept design and vice versa. For example the preliminary interviews with orthopaedic surgeons highlighted the technical 
gap for biomaterials with both strength and osteoconduction which helped guide the porous structure design towards the sparse network of pores. Secondly the specialised biomaterial design provided the inventive concept which the patent strategy was designed to protect.

As in many team situations there are benefits but times where communication breaks down due to differences in opinion. In the bone project this stemmed from different perspectives on how the group would work as a team: individuals responsible for separate components or all components developed by the group together. This could be considered beneficial as it simulates the real world environment and an objective of the course is the development of successful teams (not only in the university). Various attempts were made to manage the situation with limited success with only looming course deadlines resolving remaining issues. There was no assigned leader or organiser with authority to assert pressure, either real or from expectation, leaving the situation in limbo with no resolution (nor was there the ability to divest individuals from the group). In some respects this is appropriate since individuals are expected to be able to work independently within a postgraduate programme but the bone project may have been more successful if there was a leader with authority.

While there were slight differences, presumed here to be due the academic environment, a situation of scapegoating was created in the bone project team. Scapegoating is where an individual is faulted for the failure of the project (due to not completing work); the result is the targeted individual becomes isolated and disenchanted with the project ("Difficult Group Behaviours," n.d.). There were slight variations as in the beginning the conflict was not for the delay of the bone project's development but expectations that each person would apply equal effort to their role (thus not creating an end point where the project is finished but not all contributed).

A result of this was the basic surveying and interviewing with orthopaedic surgeons were delayed, or not completed, and were substituted by a review of medical literature (fortunately in medical there is an advantage it that problems and effectiveness of different implants are well documented). The negative is that the design process relied almost (the exceptions, key to the direction of the project, have already been discussed) exclusively on technical constraints around current biomaterials to inform the design - not on what the end users, orthopaedic surgeons, actually wanted (which for example could be non-biodegradable, i.e. titanium, implants that do not lose fixation).

The conflict within the bone project team occurred because not everyone was happy with their roles at the beginning or as the evolved of the duration of the year (as influenced by how the enterprise development around synthetic nacre developed). The original team was built around 
functional roles and personal backgrounds: engineering, biological and intellectual property, marketing and strategy. Additional responsibilities were allocated where individuals volunteered or it was deemed appropriate. The previously mentioned communication breakdown shows that this was not completely successful for the bone project team.

Through discussion with Susan Sage, a small business consultant, it was apparent that as part of the team formation the motivations and personality types of each individual should have been taken into account when dividing up the functional roles. For example a creative visionary personality type will not be happy or useful in a role that requires "dotting i's and crossing t's". The bone project team lacked an individual with a people oriented personality needed to fill the market validation role. This void created a situation where the design concept focused on the technical aspects of synthetic nacre and osteoconduction at the expense of a reduced (in scope) and slower validation cycle with the potential customers.

\section{Summary}

The structure of the course, the individual role within the team, and team dynamics are important aspects of enterprise development and influenced the direction of the bone project. As the understanding of synthetic nacre developed and changed the direction of the bone project the role evolved to match; changing from technical development to preliminary engineering design. The tools and structured used in the course biased the project towards a standalone business concept. The team dynamics influenced the design process and concept in both positive and negative ways but particularly the lack of end user feedback has stunted the validity of the biomaterial platform concept. Taking into account personality types and not just functional roles when building the team could have potentially avoided this weakness. 


\section{CHAPTER 8 CONCLUSIONS}

The role of an engineer to solve problems was, in a partial manner, confirmed for the bone project enterprise development around synthetic nacre. The objectives to develop an ideal concept for a biodegradable osteoconductive load bearing implant biomaterial design concept was completed and extended with a single material and manufacturing system utilising concepts from adaptive manufacturing.

A number of advanced engineering and design concepts, such as adaptive manufacturing and concurrent design, appear suitable for future aspects of the bone project; though being at such as early preliminary planning stage these may never be used. Interestingly there was no conscious deliberate attempt to implement these concepts; they came about as a response to the complexities, constraints, and requirements of the project. This raises the question whether advanced strategies such as these could naturally occur in engineering and enterprise development where solutions are sought for sufficiently complex problems - considering Honda and Toyota have developed similar systems independently reinforces this view.

The extended time frame for commercialisation medical applications and the very early stage development of the material itself required separating the term "solve problems" into identify, propose, and implement stages of which only the first, and to a lesser extend the second being completed during the course of the yearlong project.

These basic stages of problem solving have been transposed onto the engineering design process. Reflecting upon this showed that the basic product development cycle which included design, testing, and evaluating was not completed suggesting the bone project, even if the biomaterial concept is correct and will be successful, was a failure in terms of not being able to confirm a positive results of the design process. Without testing and evaluating the conceptual design and technical capabilities of the synthetic nacre there is no evidence to suggest whether the business case has any validity. In addition applying the objectives of lean and the MATE course, to fail quickly and provide a safe environment for failure, reinforces this judgement.

Within the enterprise development individuals worked together in a team in an attempt to complete a successful commercialisation project. The ability to work within a team and healthy team dynamics is essential and cannot be ignored despite not being core to the role discipline. The group environment effected the progress and engineering design decisions during the bone project concept; the process to form the team could have been improved. 
Through this experience a number of skills and approaches have been adapted across to different fields while acquiring specialised knowledge in hard tissue engineering. Understanding why and how the engineering design process was followed and where it failed supports the belief that in the future all effort should be made to reduce the design, test, evaluate cycle time so that it can be completed; in particular to enterprise development this should not only tests evolving technical efficacy but end user needs and desires as well.

For the biomaterial commercialisation project itself the focus for the following 6 months should be on developing the ability to create 3D porous structures - either by the current 3D fabrication method or a more traditional approach. Without the ability to create greater wall thicknesses than currently possibly with the material the targetable applications possible appear very limited. 


\section{BIBLIOGRAPHY}

Aranaz, I., Mengíbar, M., Harris, R., Paños, I., Miralles, B., Acosta, N., ... Heras, Á. (2009). Functional characterization of chitin and chitosan. Current Chemical Biology, 3(2), 203-230.

Atlan, G., Delattre, O., Berland, S., LeFaou, A., Nabias, G., Cot, D., \& Lopez, E. (1999). Interface between bone and nacre implants in sheep. Biomaterials, 20(11), 1017-1022.

Atlan, G., Balmain, N., Berland, S., Vidal, B., \& Lopez, É. (1997). Reconstruction of human maxillary defects with nacre powder: histological evidence for bone regeneration. Comptes Rendus de l'Académie Des Sciences - Series III - Sciences de La Vie, 320(3), 253-258.Avramenko, Y. (2008). Case based design: applications in process engineering. Berlin: Springer.

Azevedo, H. S., \& Reis, R. L. (2005). Understanding the enzymatic degradation of biodegradable polymers and strategies to control their degradation rate. Biodegradable Systems in Tissue Engineering and Regenerative Medicine. Boca Raton, FL: CRC Press, 177-201.

Bergsma, J. E., de Bruijn, W. C., Rozema, F. R., Bos, R. R. M., \& Boering, G. (1995). Late degradation tissue response to poly(I-lactide) bone plates and screws. Biomaterials, 16(1), 25-31.

Blank, S. G., \& Dorf, B. (2012). The startup owner's manual: the step-by-step guide for building a great company : vol. 1. Pescadero (California): K\&S Ranch.

Brunicardi, F. C., Gibbs, R. A., Wheeler, D. A., Nemunaitis, J., Fisher, W., Goss, J., \& Chen, C. (2011). Overview of the Development of Personalized Genomic Medicine and Surgery. World Journal of Surgery, 35(8), 1693-1699.

Bueter, C. L., Lee, C. K., Rathinam, V. A. K., Healy, G. J., Taron, C. H., Specht, C. A., \& Levitz, S. M. (2011). Chitosan but Not Chitin Activates the Inflammasome by a Mechanism Dependent upon Phagocytosis. Journal of Biological Chemistry, 286(41), 35447-35455.

Chang, H.-I., \& Wang, Y. (2011). Cell responses to surface and architecture of tissue engineering scaffolds. In Regenerative Medicine and Tissue Engineering - Cells and Biomaterials. InTech.

Charles, L. F., Kramer, E. R., Shaw, M. T., Olson, J. R., \& Wei, M. (2013). Self-reinforced composites of hydroxyapatite-coated PLLA fibers: Fabrication and mechanical characterization. Journal of the Mechanical Behavior of Biomedical Materials, 17, 269-277. 
Demers, C., Hamdy, C. R., Corsi, K., Chellat, F., Tabrizian, M., \& Yahia, L. (2002). Natural coral exoskeleton as a bone graft substitute: a review. Bio-Medical Materials and Engineering, 12(1), 15-35.

Difficult Group Behaviours. (n.d.). Retrieved February 19, 2015, from http://www.skillsyouneed.com/ips/difficult-group-behaviour.html

ESA. (2012, July 31). What is concurrent engineering. Retrieved February 23, 2015, from http://www.esa.int/Our_Activities/Space_Engineering_Technology/CDF/What_is_concur rent_engineering

Fernandez, J. G., \& Ingber, D. E. (2013). Bioinspired Chitinous Material Solutions for Environmental Sustainability and Medicine. Advanced Functional Materials, 23(36), 4454-4466.

Garvey, P., \& Pinto, A. (2009). Introduction to functional dependency network analysis. Presented at the Second International Symposium on Engineering Systems, Massachusetts Institute of Technology, Cambridge, Massachusetts.

Hirschhorn, J. S., McBeath, A. A., \& Dustoor, M. R. (1971). Porous titanium surgical implant materials. Journal of Biomedical Materials Research, 5(6), 49-67.

Jarvis, M. (1999). Concurrent engineering. Work Study, 48(3), 88-91.

Karageorgiou, V., \& Kaplan, D. (2005). Porosity of 3D biomaterial scaffolds and osteogenesis. Biomaterials, 26(27), 5474-5491.

Kennedy, M., M. (2004, May 6). The Toyota Product Development System. Machine Design.

Lee, K., \& Kang, K. C. (2004). Feature dependency analysis for product line component design. In Software Reuse: Methods, Techniques, and Tools (pp. 69-85). Springer.

Li, X., Min, M., Du, N., Gu, Y., Hode, T., Naylor, M., ... Chen, W. R. (2013). Chitin, Chitosan, and Glycated Chitosan Regulate Immune Responses: The Novel Adjuvants for Cancer Vaccine. Clinical and Developmental Immunology, 2013, 1-8.

Lopez-Heredia, M. A., Goyenvalle, E., Aguado, E., Pilet, P., Leroux, C., Dorget, M., ... Layrolle, P. (2008). Bone growth in rapid prototyped porous titanium implants. Journal of Biomedical Materials Research Part A, 85A(3), 664-673. 
Lu, J. X., Flautre, B., Anselme, K., Hardouin, P., Gallur, A., Descamps, M., \& Thierry, B. (1999). Role of interconnections in porous bioceramics on bone recolonization in vitro and in vivo. Journal of Materials Science: Materials in Medicine, 10(2), 111-120.

Mahmoud, M. S., \& Xia, Y. (2012). Applied Control Systems Design. London: Springer London.

Mathews, S., Gupta, P. K., Bhonde, R., \& Totey, S. (2011). Chitosan enhances mineralization during osteoblast differentiation of human bone marrow-derived mesenchymal stem cells, by upregulating the associated genes: Chitosan enhances mineralization. Cell Proliferation, 44(6), 537-549.

Morgan, L. M., Weager, B. M., Hare, C. M., Bishop, G. R., \& Smith, G. M. (2009). Self reinforced polymer composites: Coming of age. Self, 5, 13.

Munro, N. H., \& McGrath, K. M. (2012). Biomimetic approach to forming chitin/aragonite composites. Chemical Communications, 48(39), 4716-4718.

neovascularization. (2003). Miller-Keane Encyclopedia and Dictionary of Medicine, Nursing, and Allied Health (Seventh Edition).

$\mathrm{Ni}, \mathrm{M}$. (2003). Nacre surface transformation to hydroxyapatite in a phosphate buffer solution. Biomaterials, 24(23), 4323-4331.

Osterwalder, A. (2010). Business model generation: a handbook for visionaries, game changers, and challengers. Hoboken, NJ: Wiley.

Ostrowska-Czubenko, J., Pieróg, M., \& Gierszewska-Drużyńska, M. (2013). Water state in chemically and physically crosslinked chitosan membranes. Journal of Applied Polymer Science, 130(3), $1707-1715$.

Park, B. K., \& Kim, M.-M. (2010). Applications of Chitin and Its Derivatives in Biological Medicine. International Journal of Molecular Sciences, 11(12), 5152-5164.

Pereira Mouriès, L., Almeida, M.-J., Milet, C., Berland, S., \& Lopez, E. (2002). Bioactivity of nacre water-soluble organic matrix from the bivalve mollusk Pinctada maxima in three mammalian cell types: fibroblasts, bone marrow stromal cells and osteoblasts. Comparative Biochemistry and Physiology Part B: Biochemistry and Molecular Biology, 132(1), 217-229. 
Radhakrishnan, H. (2006). Solidification by drying: Effect of non-uniformities (Ph.D.). University of Minnesota, Ann Arbor. Retrieved from ProQuest Dissertations \& Theses Global.

Raghunath, J., Rollo, J., Sales, K. M., Butler, P. E., \& Seifalian, A. M. (2007). Biomaterials and scaffold design: key to tissue-engineering cartilage. Biotechnology and Applied Biochemistry, 46(2), 73.

Ranjan, R. (2011). Fate of hydroxyapatite crstals used as bone graft substitute in benign lytic lesions of long bones. Internet Journal of Orthopaedic Surgery, 18(2), 1.

Rinaudo, M. (2006). Chitin and chitosan: Properties and applications. Progress in Polymer Science, 31(7), 603-632.

Roberts, J. M. (Ed.). (2009). Cold-water corals: the biology and geology of deep-sea coral habitats. Cambridge, UK ; New York: Cambridge University Press.

Rolstadås, A., Henriksen, B., \& O’Sullivan, D. (2012). Manufacturing Outsourcing. London: Springer London.

Rouwkema, J., Rivron, N. C., \& van Blitterswijk, C. A. (2008). Vascularization in tissue engineering. Trends in Biotechnology, 26(8), 434-441.

Sepe, M. (2013, April). PART 4: Dimensional stability after molding. Plastics Technology, 59(4), 1517.

Shafiu Kamba, A., \& Zakaria, Z. A. B. (2014). Osteoblasts Growth Behaviour on Bio-Based Calcium Carbonate Aragonite Nanocrystal. BioMed Research International, 2014, 1-9.

Shahgoli, S., DDS, \& Levine, M. H., DMD, MD. (2011). Introduction and Overview of Bone Grafting. New York State Dental Journal, 77(2), 30-2.

Sherman, E. (2013, November 18). Mass Customization: Let Your Customers Have It Their Way. Retrieved February 19, 2015, from http://www.inc.com/erik-sherman/masscustomization-let-your-customers-have-it-their-way.html

Sikavitsas, V. I., Temenoff, J. S., \& Mikos, A. G. (2001). Biomaterials and bone mechanotransduction. Biomaterials, 22(19), 2581-2593. 
Sobek, D. K. I. (1997). Principles that shape product development systems: A Toyota-Chrylser comparison. University of Michigan.

Sun, K. (2011). Preparations, properties and applications of chitosan based nanofibers fabricated by electrospinning. Express Polymer Letters, 5(4), 342-361.

The Economist. (2013, September 7). 3D printing scales up. The Economist, 408(8852), 11.

Törmälä, P. (1992). Biodegradable self-reinforced composite materials; manufacturing structure and mechanical properties. Clinical Materials, 10(1-2), 29-34.

Townend, O. (2014). Managing to start: The application of lean startup and design thinking to academic commercialisation projects. Victoria University of Wellington.

Wegst, U. G. K., \& Ashby, M. F. (2004). The mechanical efficiency of natural materials. Philosophical Magazine, 84(21), 2167-2186.

Whang, K., Healy, K. E., Elenz, D. R., Nam, E. K., Tsai, D. C., Thomas, C. H., ... Sprague, S. M. (1999). Engineering bone regeneration with bioabsorbable scaffolds with novel microarchitecture. Tissue Engineering, 5(1), 35-51.

Yildirimer, L., \& Seifalian, A. M. (2014). Three-dimensional biomaterial degradation - Material choice, design and extrinsic factor considerations. Biotechnology Advances, 32(5), 984999.

Zheng, R., Tanner, R. I., \& Fan, X.-J. (2011). Shrinkage and Warpage. In R. Zheng, R. I. Tanner, \& X.J. Fan, Injection Molding (pp. 87-104). Berlin, Heidelberg: Springer Berlin Heidelberg.

Zhou, C., Ma, L., Li, W., \& Yao, D. (2011). Fabrication of tissue engineering scaffolds through solidstate foaming of immiscible polymer blends. Biofabrication, 3(4), 045003. 

APPENDIX A

\author{
BUSINESS CASE
}

BY

CHRISTINA HOULIHAN,

MICHAEL METTRICK, \&THOMAS SOBIECKI

Submitted to the Victoria University of Wellington

In partial fulfilment of the requirements for the degree of

Master of Advanced Technology Enterprise

Victoria University of Wellington 


\section{Executive Summary}

NacreTech is a material and manufacturing platform based upon research that is being developed within Victoria University of Wellington. It relies on the further development of a unique biomaterial, which has the potential to be used as a scaffold for bone growth.

The biomaterial consists of a flexible polymer matrix which is strengthened through the crystallisation of a secondary compound into the matrix surface. The combination of the matrix and the secondary compound is hypothesised to allow for a rigid, but not brittle, material with similar strength to bone. A specific porous macrostructure is considered to be crucial for supporting bone growth while retaining strength. This combination of features is considered to give the NacreTech biomaterial a competitive advantage.

Traditional methodologies are unable to create the detailed porous structure. Hence a bottom up process such as 3D printing is considered to be the best method of manufacture as it can allow the biomaterial to be tailored for many different applications

Discussed in detail is a potential initial application of the biomaterial as a bioactive bone screw. Currently most available bioactive bone screws are made with poly-lactic acid (PLA) as a base material, which results in the following identified problems: weak material strength resulting in breakage, fast degradation rates resulting in incomplete healing or holes left in the bone and harmful acidic by-products which impede healing, lack of reliable clinical data and trust by surgeons.

The bone screws produced by the NacreTech product platform are anticipated to overcome these existing problems, due to the attributes of the material. Specifically, the porous macrostructure will increase bone growth rates, while the base material reduces the rate of degradation.

The vision of the NacreTech platform is not a single market application, such as bioactive bone screws, but a range of different product lines. The ability to 3D print will enable adaptive manufacturing of the biomaterial for new applications without the expense of developing a new production process. Initially, the product line could easily expand to produce bolts, pins, plate systems, and other bioactive implants for use in orthopaedic applications.

The long term vision is the manufacture of personalised orthopaedic implants. This can be achieved due to the 3D printing which allows for the tailoring of the porosity, strength, degradation rates and design to meet the needs of the patient and the orthopaedic surgeon. 


\section{Contents}

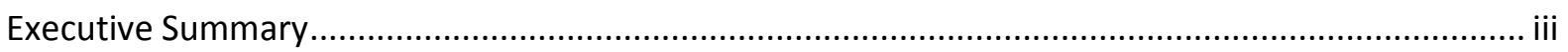

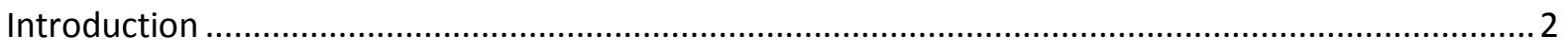

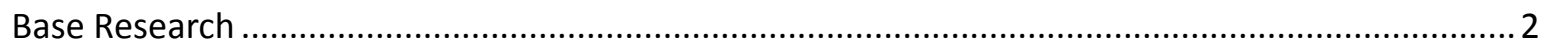

Team Summary

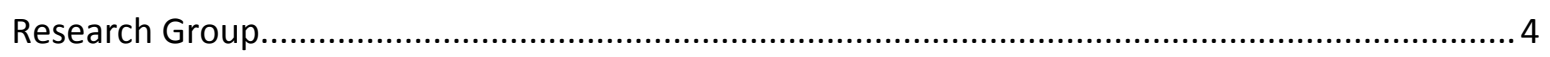

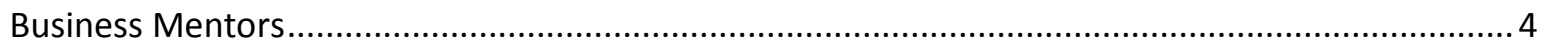

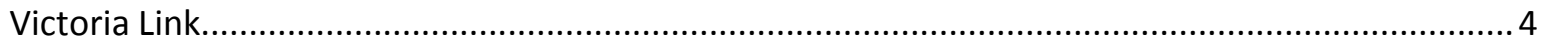

Christina Houlihan - Biological, intellectual property and regulatory aspects ................................. 4

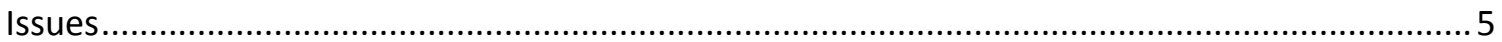

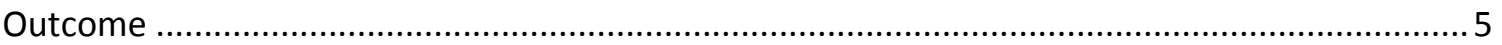

Thomas Sobiecki - Biomaterial design, mechanical, and manufacturing aspects ............................. 5

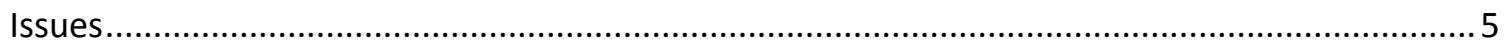

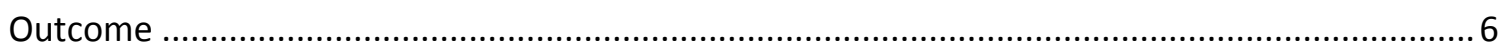

Michael Mettrick - Market research, strategy, and business model development........................... 6

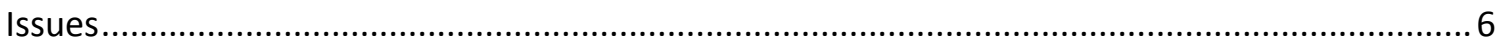

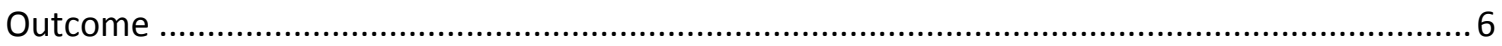

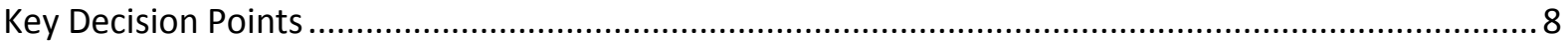

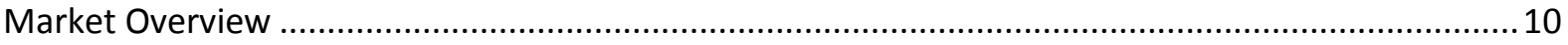

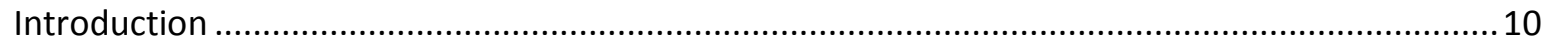

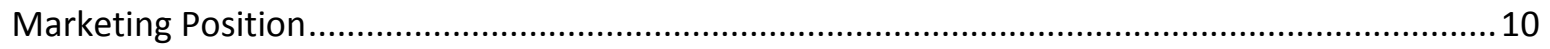

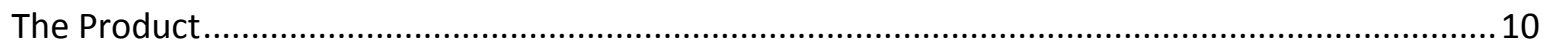

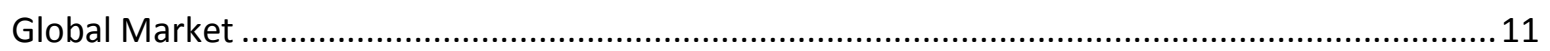

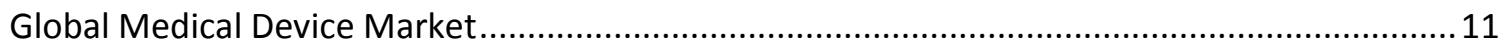

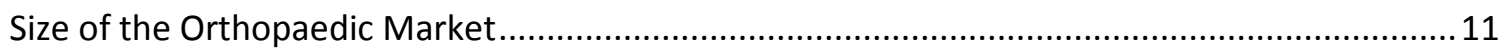

Size of the Trauma Fixation Device Market .............................................................................. 12

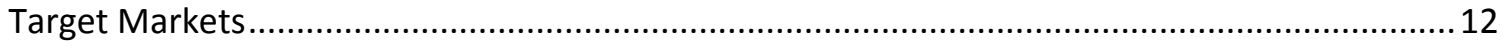

Effect on NacreTech: Analysis of Global Operating Environment ............................................... 12

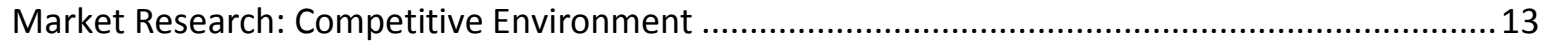

Significant Players in the USA and European Markets for Bioactive Bone Screws ...................... 13

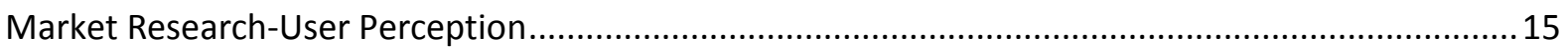

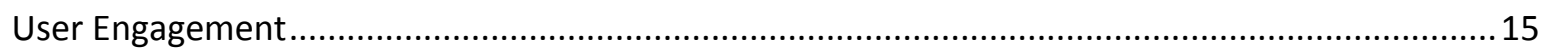

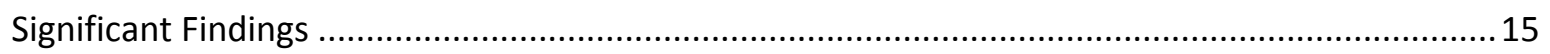

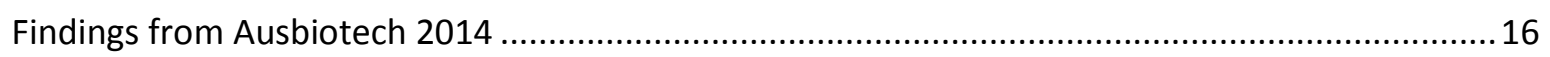




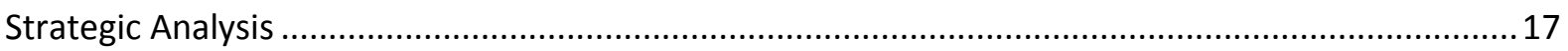

PESTEL Analysis for Bioactive Bone Screws ................................................................. 17

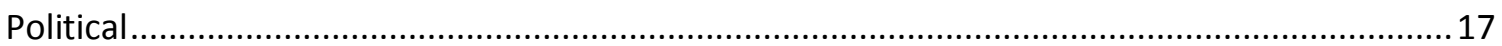

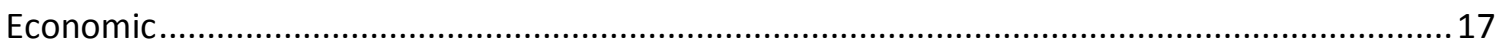

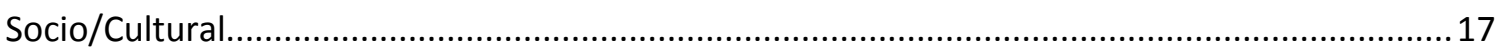

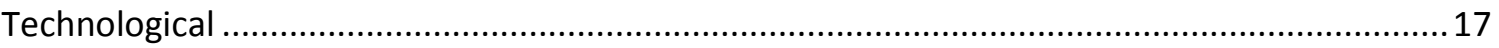

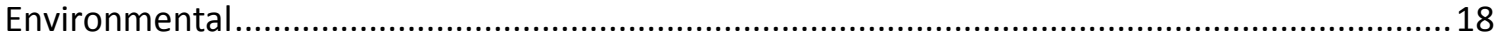

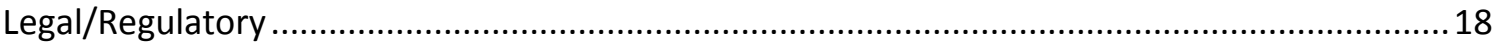

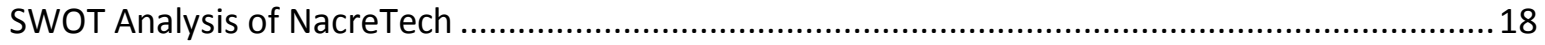

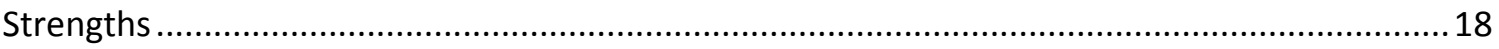

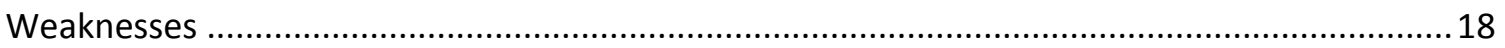

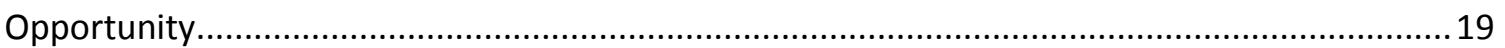

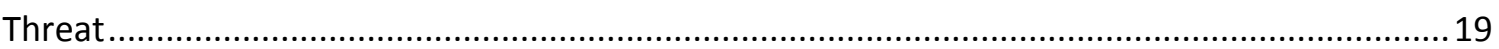

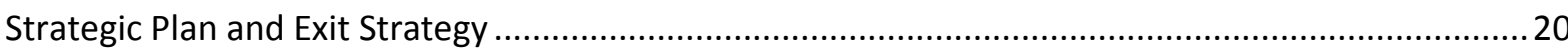

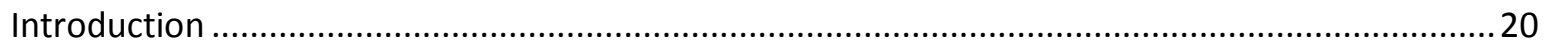

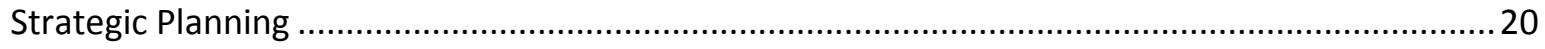

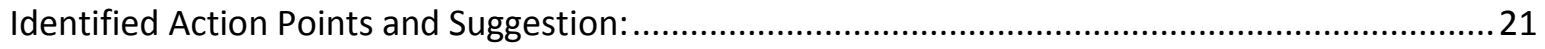

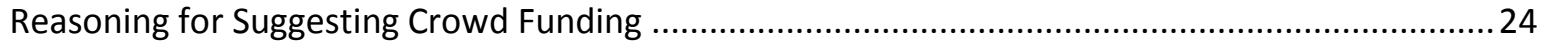

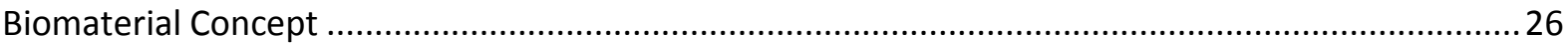

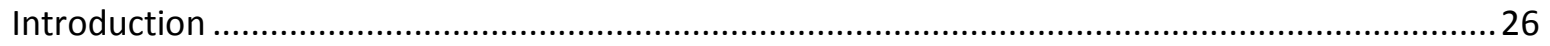

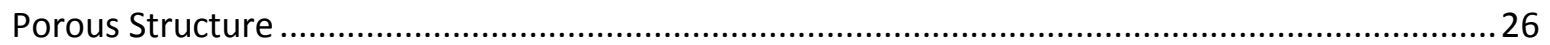

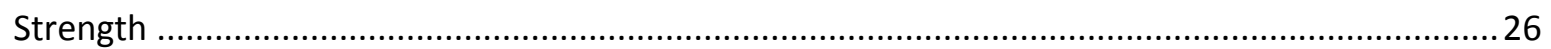

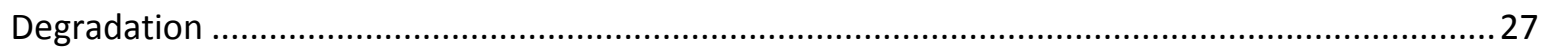

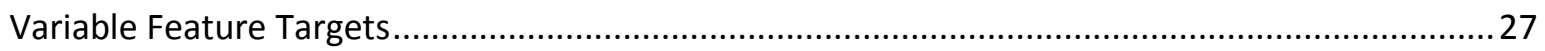

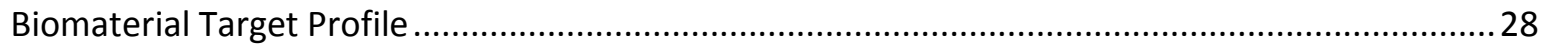

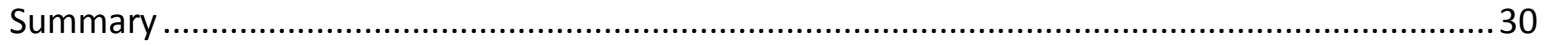

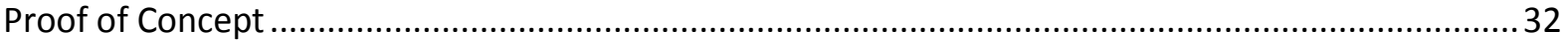

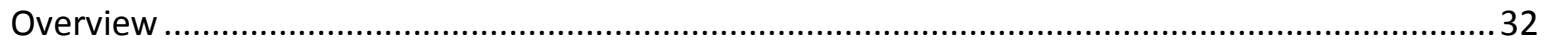

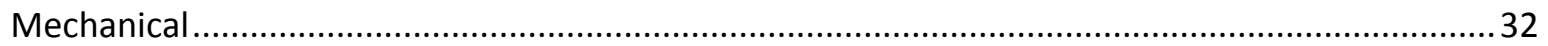

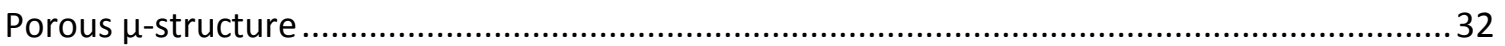

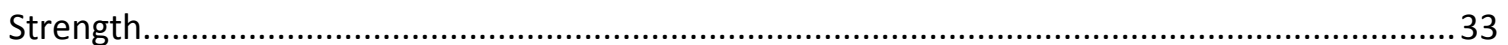

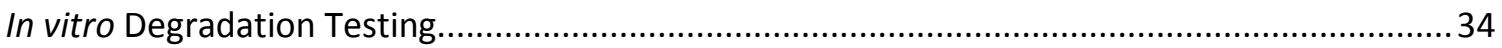

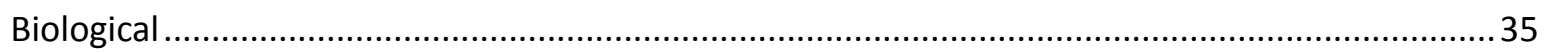

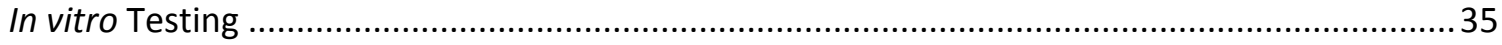




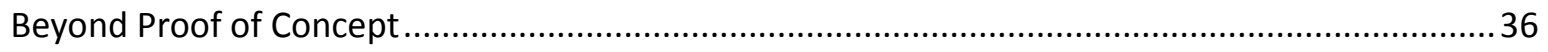

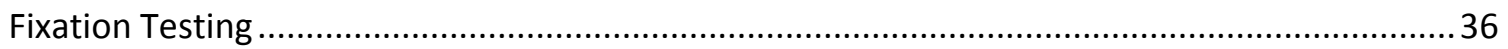

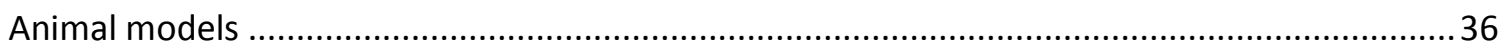

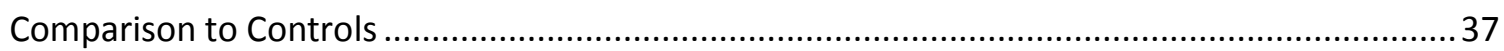

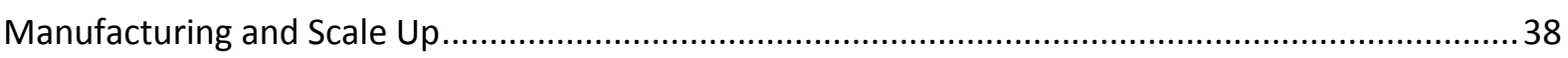

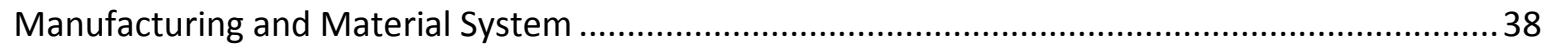

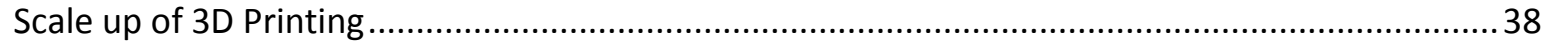

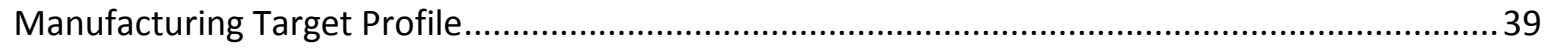

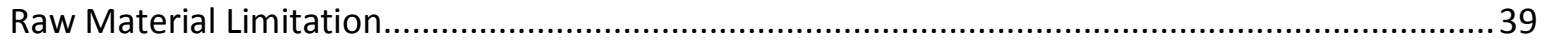

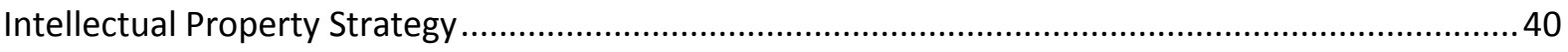

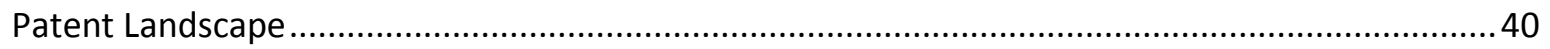

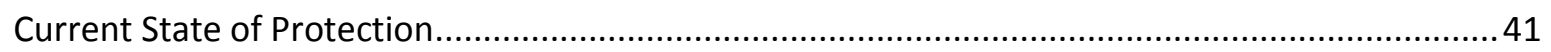

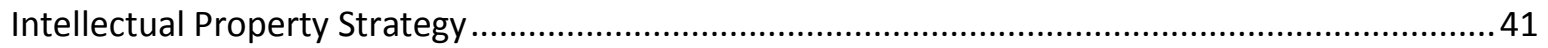

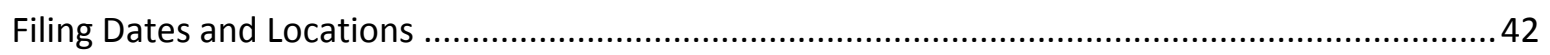

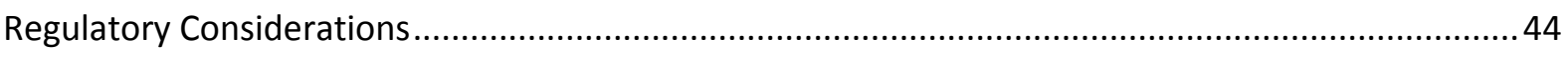

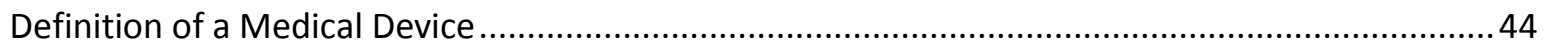

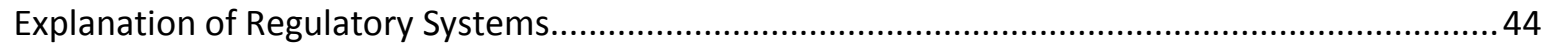

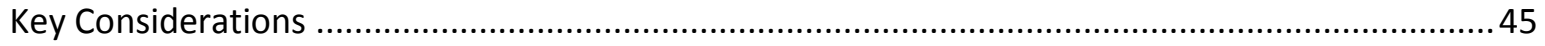

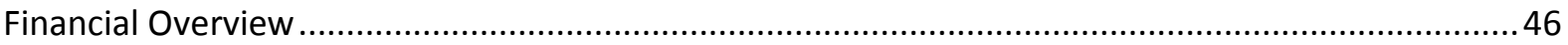

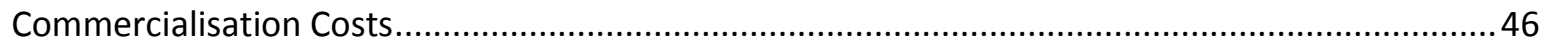

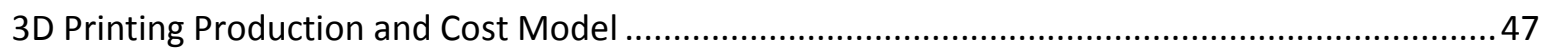

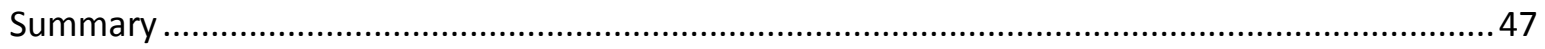

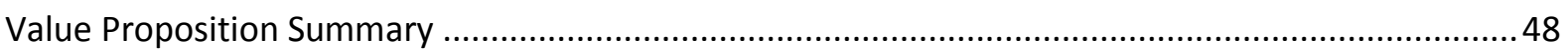

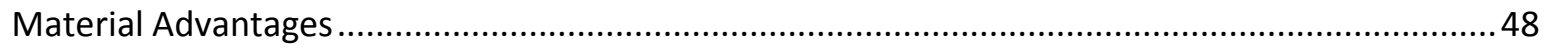

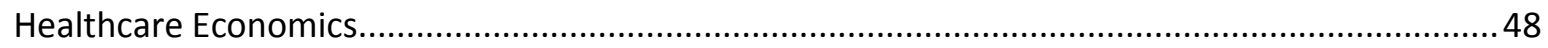

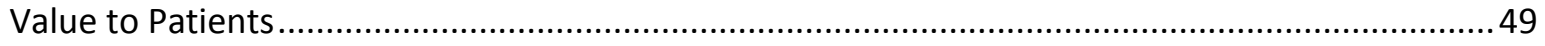

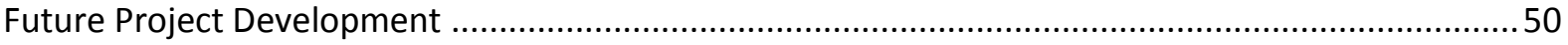

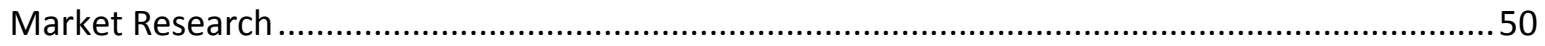

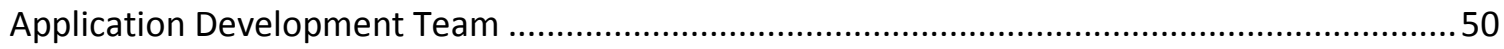

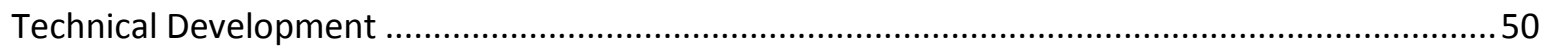

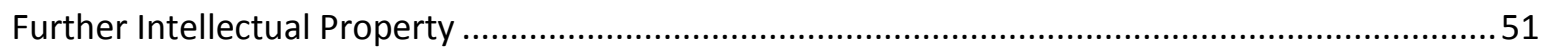

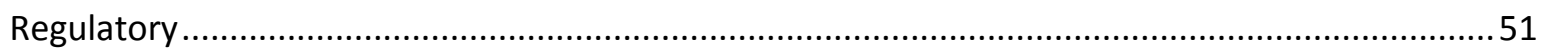

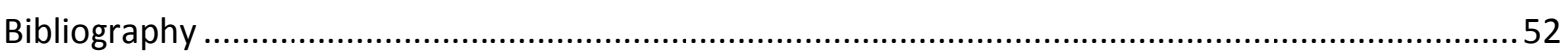




\section{List of Figures}

Figure 1: Total health expenditure expressed as a percentage of gross domestic product (GDP) for 12 industrialised economies (World Health Organization, 2014) .......................................................... 11

Figure 2 Conceptual diagram comprising different biomaterial features ............................................ 28

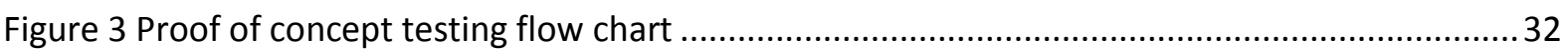

Figure 4 Image adapted from (Kherlopian, et al., 2008)................................................................ 33

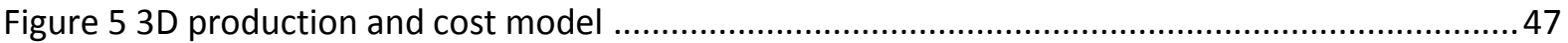

\section{List of Tables}

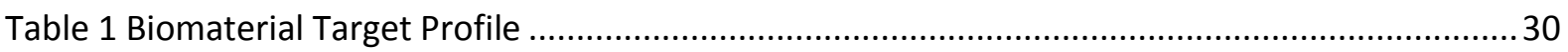

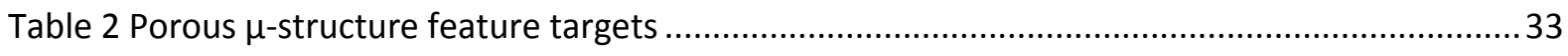

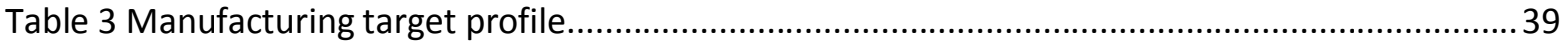

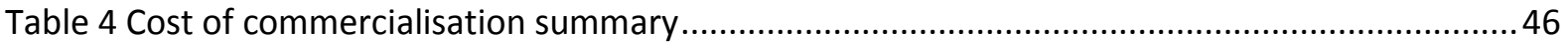




\section{Introduction}

The NacreTech biomaterial platform concept has been developed towards commercialisation as part of the Masters of Advanced Technology Enterprise (MATE). The goal of the MATE course is to provide the participants with practical experience in the commercialisation of university or other advanced research.

The initial project presented to the student participants was from the research laboratory of Professor Kathryn McGrath at Victoria University of Wellington (VUW). The research is based upon a method of producing a material referred to as synthetic nacre. A team was formed around this material with the purpose of determining a market application and planning subsequent development of the enterprise.

The purpose of this document is to provide the initial findings of the NacreTech team. It is recognised that the material is still in the early stages of development. As such, the focus of this document is providing a potential market application, the immediate next steps in the product development and initial legal considerations.

\section{Base Research}

The initial research was to mimic the chemical process by which molluscs, oysters, and mussels produce a material within their shells called nacre. Nacre is a naturally formed composite of calcium carbonate, in the form of aragonite, structural protein, and chitin; an example of the resulting material is the semi iridescent inside lining of a mollusc shell (Munro, Green, \& McGrath, 2013).

The initial research provided to the team was the method of producing a material similar to nacre which the research team called synthetic nacre. At a basic level this material is a composite of a chitin/chitosan natural polymer scaffold and calcium carbonate in the form of aragonite crystals; the primary difference from the natural form is the absence of proteins and contaminants creating a material that is potentially suitable for medical applications.

The research group developed this material using a combination of known chemical processes which resulted in the surprising and novel formation of the aragonite integrated into the polymer scaffold. Additionally a process to 3D print the chitin scaffold followed by the mineralisation has been shown to work in the laboratory. There are limitations are on the maximum wall thickness of the material which is restricted by limits of diffusion. During experimentation samples have been made in microspheres, films, and printed rings all $<1 \mathrm{~mm}$ at their maximum thickness. 


\section{Team Summary}

\section{Research Group}

The research group, as lead by Professor Kathryn McGrath, including Dr Natasha Evans provided a support role helping the teams understanding of the research and advice on concept feasibility. The research group would also be responsible for the progression of the material to meet the target product profile.

\section{Business Mentors}

The mentors, Jennifer Anderson and Melissa Yiannoutsos from Kerasi limited, provided advice to the team regarding the commercialisation of the research.

\section{Victoria Link}

Victoria Link (VicLink) is the commercialisation branch of Victoria University of Wellington. Anne Barnett is a Senior Commercialisation Manager at VicLink who supported the NacreTech team during the MATE program.

\section{Christina Houlihan - Biological, intellectual property and regulatory aspects}

The role of Christina Houlihan within the team was initially to investigate the hypothesis that the biomaterial is capable of performing its function as a biomaterial. This was envisaged to involve determining what results are needed to confirm that the biomaterial is capable of undergoing osteoconduction and that it is non-toxic, investigating who would be capable of developing and performing these tests and then potentially arranging completion of said tests.

In the second quarter of the year (June-July), it became very clear that sample was not going be able to be produced and therefore no biological testing was going to be completed this year. As a result, the role within the team changed to focus on the patent strategy and regulatory considerations. This involved taking into consideration what biological testing needs to be completed (as per the previous role) but also to investigate the longer term obstacles associated with obtaining clinical testing results. The patent strategy role involved performing a freedom to operate search (patent landscape), developing a patent strategy which aligned with the overall business strategy and considering appropriate filing dates, locations and methods. 
Issues

Due to the specialisation and experience required in the patent and regulatory industry, formulation of specific actions in order to obtain a patent or regulatory approval was not considered to be a part of the role. In addition, as the biomaterial is still in the early stages of development, it was considered too early in the commercialisation of the biomaterial to bring such specialists into the team.

Outcome

In view of the recognition of the early stage of development, the patent strategy and regulatory considerations were considered at a high level and incorporated advice from industry professionals.

Thomas Sobiecki - Biomaterial design, mechanical, and manufacturing aspects The role of Thomas Sobiecki within the team initially was to learn the process of fabricating synthetic nacre - followed by fashioning specimens and prototypes for mechanical testing as part of proof of concept. A literature review into the current orthopaedic biomaterials and characteristics of nacre identified limitations, effect, and advantages. This review influenced the decision to choose bone screws as an application and later in the development of the target product profile.

While working with the VUW research group the current early stage development status of the material was discovered. This forced a role shift to building the technical targets for the biomaterial and reducing the proof of concept testing component to developing a preliminary plan. Investigating the technical targets evolved to include much of the biological aspects of tissue engineering design and the specialised macrostructures needed for osteoconduction to occur.

As a result of understanding the tissue engineering design aspects it became apparent in the second half of the yearlong project that 3D printing, or another additive process, would be required (as opposed to being an option among many) for fabrication. This eventually led to the development of the single material and adaptive manufacturing system concept, i.e. the NacreTech product manufacturing platform. Additionally the test design recommendations around non-standard degradation were included in the proof of concept planning.

Issues

Due to the early stage development of the material the role evolved from developing specimens and prototypes for testing to developing the technical targets for the material to meet in the future. By not completing any full design, test, evaluate loops the concept and specifications have not been validated. 


\section{Outcome}

A design concept and technical targets have been developed for a biodegradable osteoconductive load bearing biomaterial which given further development the synthetic nacre material may meet. The single material and manufacturing system concept has been developed to allow targeting a larger market from the single material using 3D printing.

\section{Michael Mettrick - Market research, strategy, and business model development} Within the team, Michael Mettrick's role was to develop the understanding of the market aspects, both customer needs and aspirations, as well as identify how their needs are being met by current competitors. Once the existence of unmet market expectations had been established, the focus switched to the strategic level identifying and developing the optimal business model.

The decision by the team to focus on orthopaedic bone screws guided the primary market research and showed a need to further understand the operating environment. The primary research phase entailed end user interviews with orthopaedic surgeons and nurses. These interviews led to a second round of secondary research to investigate the findings and help the team better understand the end users viewpoints and needs. The next step was to identify the specific surgical application for NacreTech to target as its first product.

Issues

Part way through June 2014 it became evident that the material development was not at a stage where specific applications could be considered. This early stage of material development impacted the market research because we could not target a specific surgical application of the bone screw.

\section{Outcome}

Unable to ascertain the material limitations and parameters required to isolate specific material applications caused a shift in focus. Initially the intention was for gathering further detailed market research. Later the role changed to developing the business strategy and gaining further knowledge about the competitive environment. The strategy development was directed towards establishing the best foundation for NacreTech to meet the needs of the users and also the goals of the material owners (VicLink). 


\section{Key Decision Points}

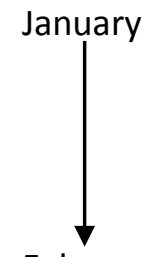

February

March

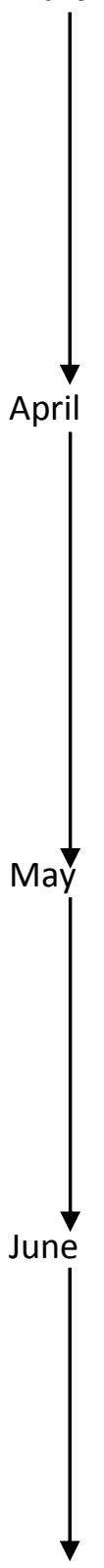

July

- 6 week intensive course

- Meeting with Anne Barnett of VicLink

- Choose project

- Developed business model canvas

- Formed team

- Defined decision making process

- Brainstormed potential material applications including: artificial reef, filtration for mining, environmental shotgun pellets, insulator, bone-carving, medical applications, tiles

- Investigation into medical market looking at orthopaedic bone substitute

- Identified strength as a potential advantage (narrowing to load bearing orthopaedic bone substitutes)

- Research indicated that there is market saturation of void fillers with approximately more than 12,000 on the market

- Cost per gram for raw materials informed decision to focus solely on medical applications

- Met with Anne Barnett- NacreTech supplied Memorandum Of Understanding (MOU)

- Research team believed mechanical testing would be done by mid-year

- Research team informed the project team about the limitations on manufacturing size leading to the decision to focus on bone screws

- Identified that the screw shape would be technically difficult to manufacture and would provide a good test case for the manufacturing process

- Laboratory observations begin regarding learning the process to synthesise the material

- Initial freedom to operate search conducted

- Primary market research started-Interviews indicated a need for active and strong biodegradable bone screws, this need is not perceived as been meet by current products, degradation rate and half-life were questioned informing aspects of the TPP investigation

- 3D printer failure delaying compression testing

- Interview with regulatory professionals regarding CE mark and FDA regulatory systems. In particular, FDA specialist suggested a drug master file or that a $510 \mathrm{k}$ may be possible

- Further secondary research is started

- National phase entry of patent

- Meeting with Professor Kathryn McGrath regarding further material development. Intention of the research team to focus on 3D printing of hydrogels

- Found further research confirming that the current screws are not up to requirements and also find out about screws becoming loose in a competitors screw- used primarily for $\mathrm{ACL}$ reconstructions

- Determined that 510k application for FDA approval was not possible due to 


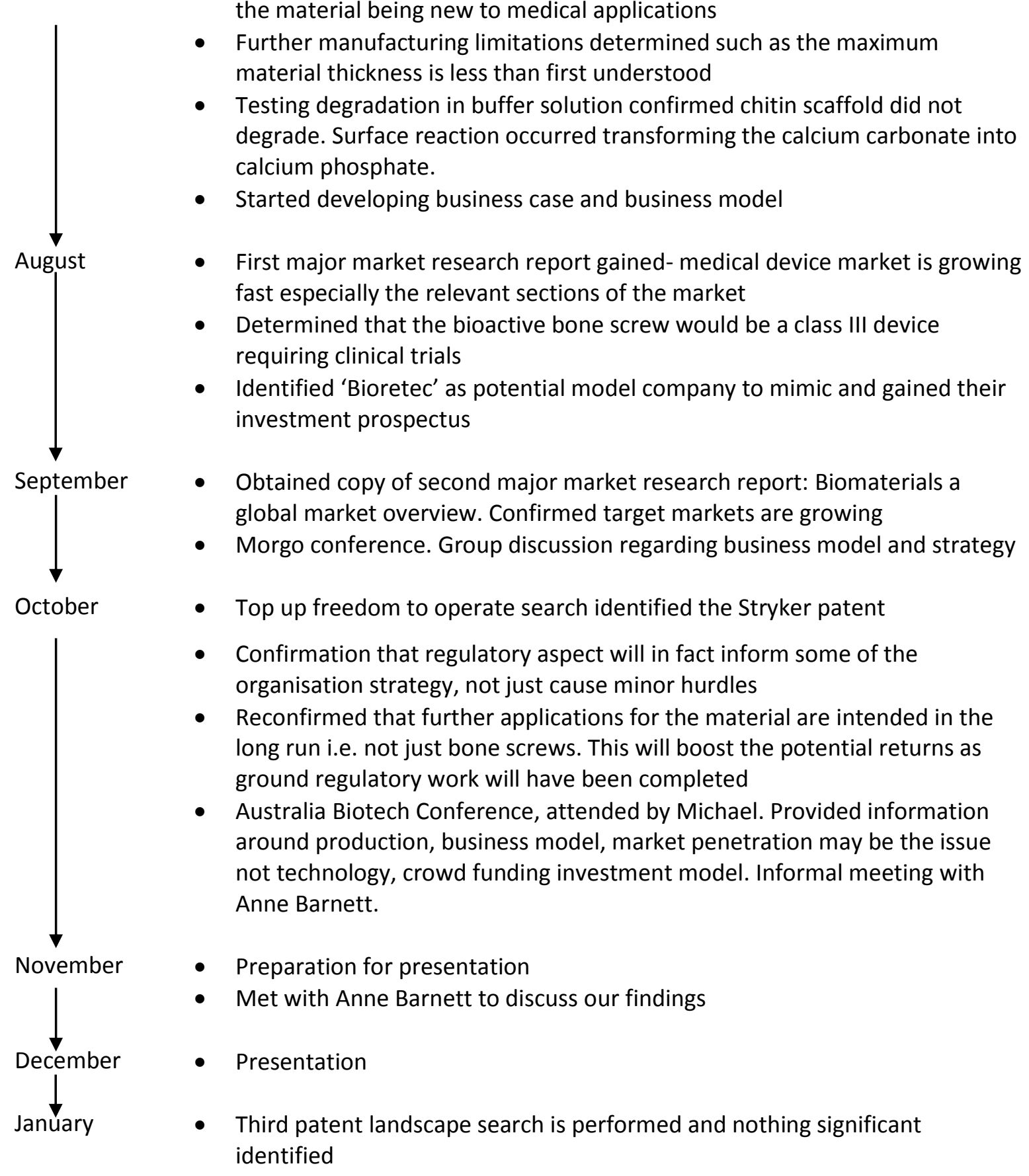




\section{Market Overview}

\section{Introduction}

NacreTech intends to bring the next generation of biodegradable materials to the orthopaedic and trauma markets. NacreTech has focused on a potential application of the biomaterial as bone screws. A combination of primary and secondary research established that current bone screws have both technical and reputational problems. Therefore, significant opportunity exists for NacreTech's biomaterial to address these market needs.

The long term goal for NacreTech is to further advance the biomaterial medical market through the production of medical devices with the ability to support natural bone recovery.

\section{Marketing Position}

Biomaterials are slowly gaining penetration into the long established medical device marketplace (Industry Experts, 2011). This slow penetration has been limited by both technical and reputational problems. Current biomaterials are used in a wide variety of medical applications from bandages to valve stents, and void fillers to glues. The production of bone screws is intended to be NacreTech's initial market niche.

The biomaterial and intended development schedule of the bone screws are planned to overcome the known technical problems with current materials and products. It is recognised that the initial price of the biomaterial bone screw will be priced above existing bone screws; however, there is the potential for reduced total cost of care and significantly improved outcomes. The intended technical development meant that there is the potential to disrupt the current marketplace for biomaterial bone screws.

\section{The Product}

The intended product of NacreTech is orthopaedic biodegradable, load-bearing bone screws. Bone screws are a form of internal fixation used by orthopaedic surgeons to support the bone during the healing process. Internal fixation is largely used in orthopaedic surgeries where serious or critical damage to the bone has occurred. NacreTech has focused on the orthopaedic and trauma markets as the primary proof of concept. Other applications such as orthodontic and Craniomaxillofacial (CMF) will be considered once material development has been completed. In addition, the specific surgical application of the bone screw will depend on the outcome of material development. 


\section{Global Market}

Globally health expenditure as a percentage of Gross Domestic Product (GDP) is increasing. This global growth is relevant because it represents a market opportunity where consumers are purchasing in increasing volumes which will support business growth. Furthermore it also presents opportunities for products that intend to reduce the overall cost, such as biodegradable bone screws that don't need to be removed. The global nature of this increase in health expenditure is shown below (World Health Organization, 2014) in Figure 1.

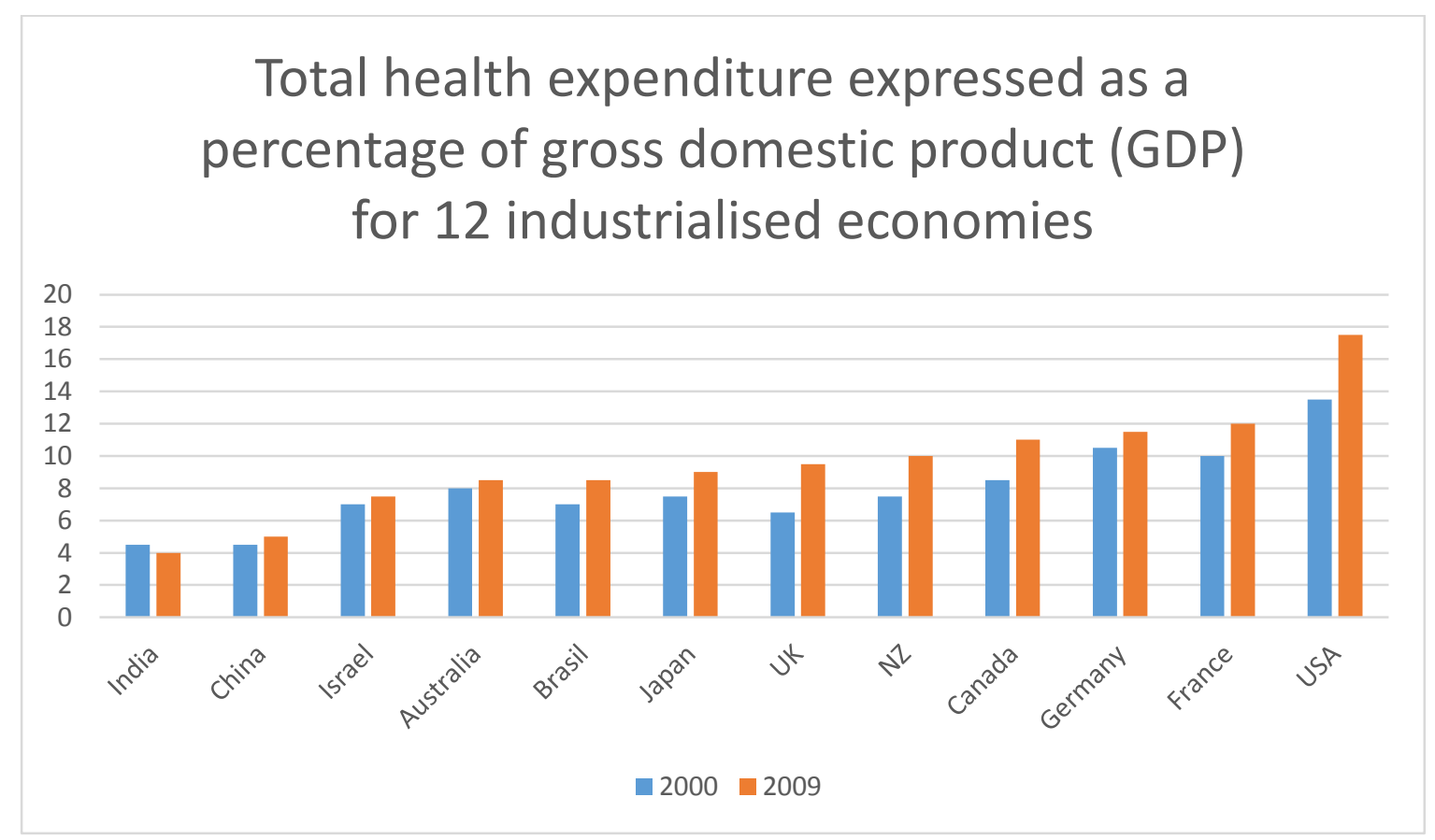

Figure 1: Total health expenditure expressed as a percentage of gross domestic product (GDP) for 12 industrialised economies (World Health Organization, 2014)

Global Medical Device Market

The market segment NacreTech is targeting is medical technology, specifically medical devices for use in orthopaedics and trauma markets. The global medical technology market was valued at over $\$ 325$ billion USD in 2014 , with the expectation that by 2018, total sales will total $\$ 440$ Billion USD (Industry Experts, 2011). This expected valuation indicates a Compound Annual Growth Rate (CAGR) of $4.5 \%$.

Size of the Orthopaedic Market Globally, the orthopaedic market accounts for approximately \$30.2Bn USD in sales turnover (Industry Experts, 2011). The CAGR of 3.1\% indicates that growth is still happening for the overall orthopaedic market. Biomaterial components of the orthopaedic market are growing fast; for example, within the United States geographic region, orthopaedic biomaterials grew at a CAGR of $11.12 \%$. 


\section{Size of the Trauma Fixation Device Market}

NacreTech is initially targeting a subset of the orthopaedics markets, the market for trauma fixation. This global market for trauma fixation is estimated at over $\$ 6.1 \mathrm{Bn}$ USD (Transparency Market Research, 2013), just under $\$ 3 B n$ USD of that is within the US market. The same United States market sees approximately 41 million visits annually to the emergency department for trauma, of these admissions almost half underwent surgery (Vanderson, 2010).

Synthes, Stryker, and Smith \& Nephew collectively dominate the Trauma fixation market supplying 70\% of overall market share (Orthoworld, 2010). A large number of SMEs account for the other $30 \%$.

\section{Target Markets}

The United States and Europe make up the largest percentage of the global market and have been identified as NacreTech's target markets. The United States accounts for $40 \%$ of the global medical technology market and Europe for 30\% (MedTech Europe, 2012).

\section{Effect on NacreTech: Analysis of Global Operating Environment}

NacreTech is looking to enter the high market growth, orthopaedic fixation market. High market growth, low market share is expected initially and the intention is to quickly grow the business to high market share within the growing market (Morrison \& Wensley, 1991). The expectation is that obtaining significant market penetration will be easier within a growing market (Solomon, 2008). 


\section{Market Research: Competitive Environment}

Approximately 400 organisations from around the world have been identified as selling or developing bioactive materials (Industry Experts, 2011). It is worth noting that the majority of these applications of biomaterials are not focused on the orthopaedic and trauma markets. Most biomaterial selling companies are focused on the larger wound care and cardiothoracic markets.

Only 37 organisations were found to target orthopaedics. Of these 37 , only seven were identified as direct competitors selling load bearing bone screws. It is also worth noting that 25 organisations were found to sell bioabsorbable interference screws, which are primarily used in anterior cruciate ligament $(A C L)$ reconstruction. Interference screws are not direct replacements for bone screws, but it is possible that some may be adapted to the bone screw market if they can meet the technical requirements.

Significant Players in the USA and European Markets for Bioactive Bone Screws

\section{Biomet}

Biomet is the largest of the identified direct competitors within the current marketplace. In 2014, Biomet had a turnover of approximately $\$ 3.2$ billion USD and targets the entire orthopaedics and trauma market (Forbes.com, 2014). Biomet is in the process of being purchased by Zimmer, a slightly larger orthopaedics device manufacturer. There are regulatory antitrust concerns about the acquisition, as the EU's antitrust regulators believe that competition would be reduced in some markets due to the size and near monopoly. Biomet has a competitive advantage resultant from their large size and considerable resources.

\section{Bioretec}

Originating from Finland, Bioretec develops, manufactures and distributes bioactive medical devices. These devices are solely within the area of bioactive fixation devices (predominantly screws and pins). Last year, Bioretec had a turnover of $\$ 681$ USD million and were in a growth phase having just completed another round of investment (Anonymous, 2014). With reference to their technical focus and business model, Bioretec are the company the team is trying to mimic; they have built almost their entire company around the concept of bioabsorbable medical devices. Bioretec also has a competitive advantage resulting from their specialisation in the area of biomaterial bone screws.

\section{Conmed}

From the United States of America, ConMed manufacture surgical devices. They specifically target general surgery, orthopaedic, sports medicine, gynaecology, gastroenterology, anaesthesiology and pulmonology. Last year ConMed had a turnover of approximately \$750 million USD (2014) (ConMed Corporation, 2014). Unfortunately for ConMed recent growth has plateaued and their corporate 
management is under threat from activist investors, whom want them to divest or be acquired by another company. ConMed has a completive advantage resulting from a company specialisation in surgical equipment.

\section{Takiron}

From Japan, Takiron are a conglomerate focused upon the use of plastics technology. Takiron had a combined turnover of approximately $\$ 600$ million last year from their diverse portfolio of industry specific companies (Markets.ft.com, 2015). We have not found recent reports of success or failure. Takiron have used their knowledge of plastics to combine the base polymer with another ceramic material. The diversity of Takiron is also a competitive advantage as they not reliant on success any one industry.

\section{Sinobiomaterials Co, Harbin Haiousi Business Co and Gunze trading limited.}

Three Chinese companies Sinobiomaterials Co, Harbin Haiousi Business Co and Gunze trading limited have been identified as selling bioabsorbable bone screws from the Chinese market. Currently only very limited information is available about each of these companies beyond product lines. We do know that they have a variety of plastics based products, and also capabilities with multiple types of plastic compositions. Sinobiomaterials Co, Harbin Haiousi Business Co and Gunze trading limited are thought to have a competitive advantage based on their locations within China where there is often considerable government support (Peng, 2012). 


\section{Market Research-User Perception}

Once secondary market research had been conducted to understand the current literature and operating environment a round of primary market research interviews were carried out. The intention of the interviews was to find out if there was an unmet need with regards to orthopaedic bone screws that could potentially be solved with the application of our material. These interviews were followed by more information gathering and a final survey to quantify the outcomes. The phased processes that we tried to follow was that recommended by literature (Adams, 2010; Zikmund, Ward, Lowe, Winzar, \& Babin, 2010).

\section{User Engagement}

Interviews were carried out with a small but highly representative sample of senior orthopaedic and trauma surgeons and senior orthopaedic nurses from Wellington Hospital in Wellington, New Zealand. These interviews were qualitative and exploratory in nature with both specific and openended questions being asked (Bryman \& Bell, 2007). The line of questioning was left largely open to enable freedom within the conversation to induce and explore interesting and useful comments (O'leary, 2010). The interviews were 30 - 45 minutes in length and conducted in May and June 2014.

\section{Significant Findings}

One orthopaedic consultant described the current bioactive materials as having limitations, they were either "Strong but not bioactive enough, or bioactive and not strong enough."

The surgeon went on to say that because of this "Bioactive materials are generally not considered for load bearing applications in adults".

This indication of a lack of market penetration for bioactive materials was very interesting to find out, mostly as a lot of the available secondary information does not support this position. Our secondary research indicated that while there had been problems with earlier bioactive materials the current generation were in general, found to meet their clinical objectives, indeed they often produced superior clinical results to the traditional metal based medical devices. Early bioactive screws were found to have problems with some screws loosening over time (Gefen, 2002). These secondary findings pointed away from a focus on the technical aspects holding back biomaterials and instead, user perceptions inhibited market penetration.

A focus upon the degradation process also became pertinent during one of the interviews. The surgeon wanted to know the degradation time and rate was including the half-life strength of our material if it was potentially going to be used in orthopaedic applications. Once mentioned it became apparent that the degradation properties would be very relevant to the user requirements. 
Desirable degradation properties have since been added to the biomaterial technical requirements based upon this conversation. Metal implants were also said generally to be removed if possible, this supported the available literature (Hanson, van der Werken, \& Stengel, 2008).

The final noteworthy findings are that surgeons are responsible for selecting the medical device that are used during surgery. The interviewed surgeons most commonly find out about new technologies from the sales people that visit them in the hospital.

A follow up survey was also created and circulated but rates of completion were so low they were statistically invalid, however useful information was still gathered that supported the findings from the conducted interviews.

\section{Findings from Ausbiotech 2014}

Attendance at the Ausbiotech 2014 conference in late October led to an informal discussion with a representative from the Therapeutic Goods Association (TGA). The TGA regulate the medical device market for Australia. The TGA representative described how he had recently been the TGA investigator for one of our direct competitors products, their bio-absorbable bone screws had flown through the clinical trial with outstanding results. Having previously investigated other similar products for the TGA he said that the ease of gaining regulatory approval was common as recent products had all been successful in the clinical trials. From TGAs perspective there are no major problems with current biomaterial products.

The TGA representative also talked about how these products have only managed small scale market penetration; not because of the technical aspects but instead because of the attitudes of the surgeons that selected the medical devices. The TGA representative described how the actions of the surgeons are justified, and described further that surgeons are specifically trained and instructed in such a way as to instil an air of self-confidence and belief that they are 'all knowing'. This air of 'knowing all' has the advantage that patients are soothed and that trust is boosted in the surgeon. According to 'Seeing what others don't' by Klein (2013) a disadvantage to 'knowing all' is that you are not open to insights and innovations. Klein describes how when someone has the perception of 'knowing all' they play it safe and relying on existing processes instead of being open to new ones. The playing it safe mentality would therefore inhibit the market penetration of cutting edge medical devices. 


\section{Strategic Analysis}

A PESTEL analysis is included to cover and identify macro-environmental factors that are expected to or may possibly impact the business (Wheelen \& Hunger, 2006). Efforts have been made so that the Strength, Weakness, Opportunity and Threat (SWOT) analysis is useful and relevant to business strategy as suggested by Piercy and Giles (1989).

\section{PESTEL Analysis for Bioactive Bone Screws}

Political

Globally there is a strong focus upon limiting or reducing overall healthcare costs. In New Zealand centralisation of bulk purchasing for public hospitals is currently occurring through Health Benefits Ltd. Bulk purchasing means that prices centrally negotiated (Summers, 1989). Funding and support is available to some start-ups in New Zealand, these businesses embody the desire to increase national GDP (Raine, O'Reilly, Teicher, New Zealand, \& Ministry of Science and Innovation, 2011). The implementation of Obamacare in the US is progressing towards collective bargaining for medical devices when they are sold within the country (Gleckman, 2014). The Trans Pacific Partnership Agreement (TPPA) which is currently under negotiation may also impact the international medical device market, currently these negotiations are secret so the potential impact is unknown.

\section{Economic}

Strong global economy. Risk of war in Eastern Europe does threaten some economic stability, as does the current Arabian conflict. Internationally there is interest in health economics and bringing down the overall cost of healthcare.

\section{Socio/Cultural}

The broad variety of international markets makes this area overly generalistic. Nonetheless, globally there is an increasing awareness of, and, acceptance for bioactive materials among the general public (medicaldevicedevelopments, n.d.). Desire for a bone substitute that is as suitable as an allograft but one that does not require additional surgeries to extract from the patient (Truumees \& Herkowitz, 1999). Globally there is an increase in health awareness(Nielsen, 2015). Indications of change with regard to the amount of evidence that is required to support device efficacy.

\section{Technological}

There are a lot of competing technologies and research institutes/companies in the field of biomaterials (Industry Experts, 2011). Therefore it is a very competitive, technologically focused marketplace. Patients were found to generally be accepting of technological advancement but medical professionals were found to be lagging(Or \& Karsh, 2009; Yarbrough \& Smith, 2007). 


\section{Environmental}

Minimal impact on the environment identified. The one exception for this is regarding the sourcing of large quantities of chemically uniform chitin for the manufacturing process.

\section{Legal/Regulatory}

Proposed changes to the Food and Drug Administration (FDA) and EU medical device regulatory systems may increase examination costs to prove efficacy and safety of proposed devices.

\section{SWOT Analysis of NacreTech}

\section{Strengths}

- Novel material has had a patent application that has been filed in multiple markets, this application creates the foundation for the intellectual property strategy.

- Development work is already underway to meet the Biomaterial Technical Properties (BTP).

- Intended products look to meet the needs of the global interest in health economics and overall cost/benefit analysis.

\section{Weaknesses}

- Lack of funding relative to the required investment.

- Current lack of dedicated team taking over once the MATE program concludes.

- NacreTech does not currently have a presence in the market.

- NacreTech does not currently have a science advisory group, or board of governance.

- Costs required to meet the BTP are expected to be considerable. Development costs are also expected to be considerable.

- May not be possible to meet the BTP within a reasonable time as it relies on a number of factors aligning.

- Current base material has not been used in internal medicine, potentially causing delays or blockages. Proof of concept testing and clinical trials are expected to be required, as are the associated costs.

- Possibility that even after, considerable development the current material may not be able to meet the requirements of the BTP.

- Currently only one single patent lodged to protect the material from imitation and theft.

- Additional capabilities and resources are needed within the development team.

- An inexperienced team.

- Physical distance from intended markets. Coming from New Zealand but targeting markets on the opposite side of the world.

- End product is expected to cost more than alternative products, especially metallic screws. 


\section{Opportunity}

- Market research indicates global demand would be considerable for the type of product proposed.

- Limited number of identified direct competitors.

- $\quad$ BTP for massively scalable product/s.

- Opportunity for multiple spin-offs or pivots within the medical device market.

- Multiple potential markets.

- Chosen marketplaces are undergoing considerable growth.

- Market research shows potential influential consumers are enamoured with the concept.

- Potential to replace current biomaterial screws.

- First mover advantage with 'new generation' biodegradable material (Markides Constantinos \& Sosa, 2013). A recent patent could potentially threaten this and enable second mover advantages instead.

- Build relationships with potential customers during the product development process. Surgeons whom are involved with the development will have increased buy-in as well as providing legitimacy and credibility to the development process. As indicated by the market research, surgeons select the devices that they use therefore purchasing behaviour will be influenced.

- Opportunities to licence the material to leading medical device manufacturers.

- Opportunity to expand patent portfolio to strengthen legal position.

Threat

- Another incumbent company is preparing to enter the market with a similar product material.

- Imitation.

- Incumbents are generally large established companies.

- Potential for material scarcity as supplier specific properties are expected.

- Biomaterial screws currently have a poor reputation.

- Potential that medical screws used for other applications may be adapted and fill our market niche.

- Organisation that owns material (VicLink) has other projects that will be competing for attention and finance.

- High regulatory and development costs are expected. 


\section{Strategic Plan and Exit Strategy}

\section{Introduction}

Strategically there are a few incoming decision points and suggestions at both the strategic and operational levels. Each decision point could lead NacreTech in a variety of different directions. The overarching strategy being suggested is the dynamic model of strategy as proposed by Moncrieff (1999). In essence the mission of NacreTech's strategy is value creation; it is believed that through the use of a dynamic' model that the maximum value can be created. The dynamics model uses a combination of both deliberate-planned processes and also emergent adaptions to the current operating environment.

\section{Strategic Planning}

As proposed by Moncrieff, the strategic intent (also known as planned strategy) is a combination of the vision of senior management/stakeholders, their analysis of both current resources and capabilities and the current operating environment. The analysis, the vision and the resultant plan are all affected by the assumptions and beliefs of the person doing the analysis. It is the implementation of the plan that leads to both new emergent strategies and strategic learning. This strategic learning in turn influences the future strategic intent as well as emerging opportunities. In essence the strategic plan for NacreTech is to create a plan, implementing it and learn from how it worked as well as integrating new information.

It is also worth noting that since NacreTech is intending to sell a medical device, medical devices generally have requirements such as clinical trials and regulatory approval. These requirements as outlined within the regulatory section and are expected to dictate some of the specific milestones for NacreTech. It is within these milestones, that the above strategy is primarily intended to influence NacreTech's overall direction.

It is recognised that the key impeding decision point and associated suggestion depends on the choice of business model. There are two different business models that are primarily being considered, licensing and independent spin-off subsidiary. The suggested path for NacreTech is to pursue both of these options sequentially. If it is possible at a relatively early stage of material development to license the material to a company operating in a different market segment, then it is recommended that NacreTech capitalise on the market opportunity. Licencing at a relatively early stage will limit potential risk and curtail the investment and reduce the time to see a return on investment. 
If licencing is unsuccessful or considered undesirable, it is recommended to follow the traditional biotech development pathway. The traditional pathway would have NacreTech launch as an independent company to commercialise and sell products from the developed biomaterial platform. The exit plan for this longer pathway is to sell the firm to a large biotech company. The alternatives are to list on the share market or pursue an acquisition by a venture capital organisation. As discussed below raising a portion of capital through targeted 'crowd funding' to support the spin-off may be desirable, as long as it does not inhibit other forms of investment.

\section{Identified Action Points and Suggestion:}

\section{Governance and Science Advisory}

The intention here is to create a team to support the translation from science lab to commercial reality. A board of governance is suggested to be made from the stakeholders who have vested interests in NacreTech (IP owners, investors, VicLink etc.). Furthermore a science advisory panel should be made up from subject matter and commercialization experts.

\section{Develop Product and Capabilities in-house, or find external resources}

This project relies upon the commercialization of science. In particular further research and development is needed to meet the specific requirements of the biomaterial target profile. Currently development is underway within Victoria University of Wellington, but offsite development by a third party may be required. The decision to outsource development or not needs to be made in conjunction with decision 4 and 5 .

\section{Identification of a Physical Location for Development and Production}

Manufacturing is envisaged to rely on 3D printer technology. 3D printer technology can reduce the costs associated with the expansion of a product line. Scalable 3D printer technology can limit labour costs. Instead of 'racing to the bottom' looking for cheap labour in the international manufacturing market there is the ability to keep production physically located with development teams. This locality means the processes used in manufacturing are able to be continuously improved as new developments are realised (Ashkenas, 2012). The down side is finding a suitable location that has both development and production space available.

\section{Time Frame Considerations}

The key action to be made with regards to the future of NacreTech is deciding the timeframe for realizing the return on investment, in particular the choice of exit strategy. Both shortterm and long-term options are viable. The pathway for short-term return on investment 
focuses on licensing of the material to interested parties. The alternative long-term pathway traces the normal business creation process that most biotech start-ups follow. The longterm pathway is expected to require full development of both the technology and the business. The longer pathway means greater overall value is expected to be created but it is expected to take longer to see these significant return on investment. Return on investment is expected to take the form of dividends to shareholders or returns from being acquired by another competing company. Five and six below expand upon these two pathways.

\section{Licencing Business Model}

Licensing of the technology to a medical device maker may be possible to either one that wants to develop capabilities in next generation biomaterials, and already has compatible capabilities, or one that has resources they want to defend.

It has been identified that the largest orthopaedics company (Stryker) has recently patenting a chemically similar material, this application has opened a range of strategic options to NacreTech. We have identified two primary avenues of action. The first option is to license the use of our material to one of Stryker's competitors, these companies will be wanting to remain competitive with the newer generation of materials; this will enable fairly early acquisition of the material. At a slightly later stage licensing of the material directly to Stryker may be possible. Our material would enable Stryker to strengthen their position with regards to diversity of resources/capabilities and also stop their competitors from having similar capabilities. The licensing of the materials to one or more companies within the medical device market can be seen as the safe option; a return on the investment and research can be realized with minimal risk.

\section{Extrapreneurship Business Model}

Conversely there is the longer term, high risk, high reward option. This entails setting up NacreTech as a subsidiary/spin-off company to directly compete within the medical device market (Johnsson \& Hagg, 1987). This means further investment into developing the entire supply chain from development and design all the way though to sales and reimbursement. The intention is to emulate the success of Bioretic within the marketplace for bio-absorbable bone screws. It must also be mentioned that licensing of the material to medical device company or companies does not rule out the viability of a spin-off company but does affect the company tactical options it is worth remembering that network effects could potentially support the business (Robinson \& Stuart, 2006). 
If a business is created to realize the potential of the material, the recommendation is to pursue alternative business models such as 'crowd funding' for a small portion equity (Belleflamme, Lambert, \& Schwienbacher, 2013). Bioabsorbable bone screws have traditionally had problems with market penetration. Early generation products caused problems to the reputation of bioabsorbable bone screws (see market research), there is also indications that because of the reputation of the earlier generation bone screws the newer 'better' bone screws are having trouble with market penetration. One of the intentions with the suggestion of crowd funding is to aid market penetration. Further reasoning for suggesting crowd funding is given in the section below.

\section{Alternative Applications}

With the extrapreneurship business model it is expected that eventually there will be the opportunity to diversify the product range beyond just bone screws. The recommendation is that this step in growth for the business is not rushed and instead the focus is retained until the organisations resources and capabilities can organically support the expansion and diversification (Schaper, Volery, Weber, \& Lewis, 2011). 


\section{Reasoning for Suggesting Crowd Funding}

Interviews indicated that there is still an interest in the concept and the possibility of better healthcare that biodegradable bone screws offer. There may be an opportunity such as crowd funding to capture this interest and use it to help fund and develop the venture as well as aid development of the products that the market wants (Belleflamme et al., 2013).

The concept of crowd funding is inspired by the success of 'BrewDog' an independent craft beer brewery from Scotland. BrewDog successfully managed to combine the collection of investment/funds and also increased the vested interest of consumers as well as influencing their purchasing behaviour (Boyce, 2013). Both the investment and customer behaviour modification are beneficial to the business. BrewDog managed to achieve this is by crowd funding a small proportion of the equity in the business to consumers. Crowd funding has supported and enabled BrewDog during extremely rapid growth, averaging 285\% growth annually over the past 5 years (Brinded, 2014).

This concept of crowd funding is intended to bring a number of benefits (Ahlers, Cumming, Guenther, \& Schweizer, 2012). There is the intention to target professionals within the medical industry (specifically surgeons) with this application of crowd funding. The reasons for this specific targeting are multiple. Firstly this will facilitate engagement with surgeons who have a vested interest in the success of the project, this engagement will enable testing and development with intended users giving them a product made to their specifications and needs. User engagement will increase the legitimacy of the product and increase awareness within the target market (Suchman, 2012). The ethical implications and potential conflict of interest arising from these prospective relationships would also need to be addressed and developed so that the integrity of all parties can be maintained (Torr-Brown, 2013).

The desire is to engage with surgeons for more than just the standard development process. This engagement will boost their perception of NacreTech and associated product lines. By increasing communication and overall legitimacy with the surgeons, market penetration may increase at a faster rate than that achieved by the incumbents in the market. Finally surgeons are traditionally high net-worth, but time poor, and an investment of this nature is intended to tap into both their financial and philosophical aspirations (helping patients) as well as using their specialist knowledge.

The main concern identified with crowd funding is in regard to future investment where venture capital may be put off by the more complicated ownership structure. This concern helped reinforce the specific targeting of the investments, the hope is that by targeting the end users in such a way the positive benefits will outweigh the negatives. The second way to reduce unintentional harm to 
the business from crowd funding is to limit the size of this type of funding to specific and small amounts of total equity. 


\section{Biomaterial Concept}

\section{Introduction}

To develop a biomaterial suitable for biodegradable load bearing osteoconductive orthopaedic implants the material needs to be developed from its current status to meet the target product profile. Here is an explanation of the complexities faced to reach a number of key targets and the adaptive material concept. This is followed by a detailed table of specific targets and the set of current limitations affecting the target product profile.

\section{Porous Structure}

For the biomaterial to gain high quality osteoconductive properties the porous macro structure needs to meet certain targets for pore size and interconnectivity. There is an ideal macro pore size of 200 to $300 \mu \mathrm{m}$ (though there is disagreement in the literature (Zhou, Ma, Li, \& Yao, 2011)); if the pores are too small then the osteoblast cells may block passage further into the biomaterial's pore network; if the pores are too large then the cells may treat it as a flat surface instead of a 3D structure and the strength of the material is drastically compromised (Zhou et al., 2011).

A material with isolated pores is not sufficient as they need to be connected to each other - that is have open and conductive pores - to allow blood vessels to propagate through the biomaterial (Rouwkema, Rivron, \& van Blitterswijk, 2008). As bone growth is a very intensive process these blood vessels help determine how osteoconductive the biomaterial is by supplying nutrients; the more interconnections there are the better the result.

\section{Strength}

To meet the requirements to act as a biomaterial for load bearing implants the strength of the material needs to have an upper limit of $230 \mathrm{MPa}$ in compression; this is the approximate maximum strength of cortical bone and required for load bearing implants (Pilia, Guda, \& Appleford, 2013). Due to bone being adaptively responsive to force or load (Sikavitsas, Temenoff, \& Mikos, 2001) the strength should be near, in a similar manner to pore size, an ideal point in the middle; too strong and the biomaterial will cause stress shielding, where the surrounding natural bone is reabsorbed, as metal implants do currently; too weak and breakage will occur.

Current biomaterials on the market, such as Biomatlante's MBCP Wedges ("MBCPTM Wedges Biomatlante," n.d.), gain good osteoconductive properties by directly increasing porosity; more and more pores are squeezed into the same volume until they overlap creating interconnectivity. However this complicates reaching strength targets since there is an inverse relationship between 
porosity and strength - the more porosity, and improved osteoconduction, the less strength and vice versa (Karageorgiou \& Kaplan, 2005).

The proposed method to help lessen the effect of this relationship is to design a sparse network of connected pores. Consisting of pores connected by narrow tunnels (50 to $100 \mu \mathrm{m}$ in diameter) this will help the biomaterial retain more material while still having highly interconnected pores for the purpose of osteoconduction. How successful this porous macro structure is has not been tested within the literature reviewed for this report.

Combining strength and porosity requires balancing two inversely related properties with their own individual ideal targets.

\section{Degradation}

For the implant to be absorbed by the human body over time it needs to be biodegradable - this means the strength of the biomaterial changes over time as well. The rate or profile at which this degradation occurs is a key property that needs to be balanced with strength and porosity; they are all interrelated variables. As for strength and pore size there is a target rate of degradation in the middle; too fast and the new bone will grow incorrectly and be easily damaged; too slow and the biomaterial will impede the healing process. The ideal situation would be where the rate of degradation matches the rate of new bone growth within the patient (Raghunath, Rollo, Sales, Butler, \& Seifalian, 2007).

Joining all the above features:

For good osteoconduction there is an ideal porous structure required in the biomaterial. This macro structure inversely affects strength which has an ideal target as well. Due to degradation the strength of the biomaterial changes over time; there is an ideal rate at which this occurs.

\section{Variable Feature Targets}

There is a balancing act between the three properties to reach an optimal point compromising osteoconduction, strength, and degradation for a given application. This is achieved in some way by all biodegradable osteoconductive biomaterials available and a key target for the synthetic nacre is to improve on this by allowing the optimal point to be adapted, or changed, as required by surgeons and patients (Figure 2). 




Figure 2 Conceptual diagram comprising different biomaterial features

Gaining this ability is an important improvement since each optimal point is only useful for a limited number of applications and patient types as illustrated by (Lew, Othman, Ishikawa, \& Yeoh, 2012) regarding the various bioceramics used in the body. Depending of the type of implant, bone, and patient the ideal targets for porosity, strength, and degradation will change. A bone screw for a leg bone will have different optimal point balancing strength and porosity than a bone screw for an arm repair surgery. The rate of new bone growth changed depending on the age of the patient; for example the ideal rate of degradation for an implant in a 50 year old will be different than that for a 20 year old.

In summary enabling tailored material properties for individual implant and patient types will support a broad range of orthopaedic implant applications; turning synthetic nacre into a platform biomaterial.

\section{Biomaterial Target Profile}

The target profile has been developed to detail the feature parameters which the biomaterial should have to allow for good osteoconduction and strength. It has been established from medical literature research articles and analysis of competing products. While it is not considered to be a set measure or comprehensive list the porous structure and mechanical targets include the key aspects which should not be ignored. 


\begin{tabular}{|c|c|c|}
\hline \# $\quad$ Feature & Target & Minimum \\
\hline \multicolumn{3}{|l|}{ Porous structure* } \\
\hline Interconnectivity & $100 \%$ via $\sim 50 \mu \mathrm{m}$ tunnels & $\begin{array}{l}>70 \% \text { via } 40-100 \mu \mathrm{m} \\
\text { channels }\end{array}$ \\
\hline Macro pores & $200-300 \mu \mathrm{m}$ & $150-500 \mu \mathrm{m}$ \\
\hline Micro pores & $\begin{array}{l}\sim 5 \mu \mathrm{m} \text { pores within the } \\
\text { struts between macro } \\
\text { pores }\end{array}$ & $\sim 1-10 \mu \mathrm{m}$ \\
\hline Wall thickness** & $200-400 \mu \mathrm{m}$ & $<400 \mu \mathrm{m}$ \\
\hline \multicolumn{3}{|l|}{ Mechanical targets } \\
\hline $\begin{array}{l}\text { Elastic modulus (Joukainen, } \\
\text { 2008) }\end{array}$ & $\begin{array}{l} \pm 1 \mathrm{GPa} \text { of target bone area } \\
\text { ranging from } \sim 10-30 \mathrm{GPa} \\
\text { for cortical bone }\end{array}$ & $\begin{array}{l}>10 G P a \text { to improve on } \\
\text { synthetic plastics and } \\
<<200 G \text { a to improve on } \\
\text { stainless steel }\end{array}$ \\
\hline $\begin{array}{l}\text { Compressive strength (Pilia, } \\
\text { Guda, \& Appleford, 2013) }\end{array}$ & $\begin{array}{l} \pm 10 \mathrm{MPa} \text { of target ranging } \\
\text { up to } \sim 230 \mathrm{MPa}\end{array}$ & $\begin{array}{l}\text { It will be dependent on the } \\
\text { final application but } \\
>150 \mathrm{MPa} \text { will improve on } \\
\text { competing products. }\end{array}$ \\
\hline Tensile strength*** & $130 \mathrm{MPa}$ & $\begin{array}{l} \pm 20 \mathrm{MPa} \text { to match bone and } \\
\text { be on par with PEEK } \\
\text { material (Katti, 2004). }\end{array}$ \\
\hline Shear strength*** & $50-65 \mathrm{MPa}$ & $\sim 40-100 \mathrm{MPa}$ \\
\hline Fatigue (Teoh, 2000) & No susceptibility to fatigue & $\begin{array}{l}\text { Strength under shear stress } \\
\text { remains above } 40 \mathrm{MPa}\end{array}$ \\
\hline \multicolumn{3}{|l|}{ Surface properties } \\
\hline $\begin{array}{l}\text { Wettability (Vandrovcová \& } \\
\text { Bacakova, 2011) }\end{array}$ & Strongly hydrophilic & $\begin{array}{l}\text { Sufficient for cells to } \\
\text { propagate during } \\
\text { osteoconduction testing }\end{array}$ \\
\hline $\begin{array}{l}\text { Topography (Vandrovcová \& } \\
\text { Bacakova, 2011) }\end{array}$ & $\begin{array}{l}\text { Nanoscale }(100 \mathrm{~nm}) \\
\text { surface roughness of } \\
\sim 40 \mathrm{Ra}\end{array}$ & Nanoscale roughness \\
\hline \multicolumn{3}{|l|}{ Thermal properties } \\
\hline $\begin{array}{l}\text { Thermal contraction/expansion } \\
\text { coefficient (Holmes, 2011) }\end{array}$ & $\begin{array}{l}\sim 27 \times 10^{-6} \mathrm{~mm} / \mathrm{C}^{\circ} ; \mathrm{Or} \\
\text { near that of the } \\
\text { surrounding bone. }\end{array}$ & $\begin{array}{l}\text { Similar enough not to } \\
\text { displace set bone } \\
\text { (application specific). }\end{array}$ \\
\hline $\begin{array}{l}\text { Thermal conductivity (k) } \\
\text { (Holmes, 2011) }\end{array}$ & $\begin{array}{l}0.41 \text { to } 0.510 \text { or similar to } \\
\text { the surrounding bone }\end{array}$ & $\sim 0.13$ or similar to PLA \\
\hline \multicolumn{3}{|l|}{ Degradation } \\
\hline Strength half-life & $\begin{array}{l} \pm 2 \text { weeks over a range } \\
\text { with an upper limit of } 12 \\
\text { weeks - based off the } \\
\text { enzymatic hydrolysis } \\
\text { taking twice as long to } \\
\text { degrade the biomaterial } \\
\text { vs. non-enzymatic } \\
\text { hydrolysis (Venkatesan \& } \\
\text { Kim, 2010). }\end{array}$ & $\begin{array}{l}>6 \text { weeks or the current } \\
\text { strength half-life of } \\
\text { competing materials }\end{array}$ \\
\hline $\begin{array}{l}\text { Debris released (Böstman \& } \\
\text { Pihlajamäki, 2000) }\end{array}$ & 0 & $\begin{array}{l}\text { Slow enough that an adult } \\
\text { body can absorb the }\end{array}$ \\
\hline
\end{tabular}




\begin{tabular}{|c|c|c|}
\hline & & $\begin{array}{l}\text { released debris before it } \\
\text { builds up which can cause a } \\
\text { cascade inflammatory } \\
\text { reaction }\end{array}$ \\
\hline \multicolumn{3}{|l|}{ Specific targets for implant types } \\
\hline $\begin{array}{l}\text { Screw coaxial torsion strength } \\
\text { (dependent of design and screw } \\
\text { diameter) }\end{array}$ & & $\begin{array}{l}\text { Sufficient, when combined } \\
\text { with specialised tools, not } \\
\text { to break during insertion. } \\
\text { For example ASTM F543 } \\
\text { requires } 3.5 \mathrm{~mm} \text { metal } \\
\text { screws to withstand } 2.3 \mathrm{Nm} \text {. }\end{array}$ \\
\hline $\begin{array}{l}\text { Fixation strength (dependent of } \\
\text { screw design and application) }\end{array}$ & & $\begin{array}{l}>1200 N \text { for interference } \\
\text { screws (Kousa et al., 1995) }\end{array}$ \\
\hline Plate stiffness & $\begin{array}{l}\text { Target bone resist } 50 \% \text { of } \\
\text { force after initial healing } \\
\text { period }\end{array}$ & \\
\hline $\begin{array}{l}\text { Plat controlled plastic } \\
\text { deformation }\end{array}$ & $\begin{array}{l}0 \text { - targeting locking plates } \\
\text { as unlikely to have plastic } \\
\text { deformation with ceramic } \\
\text { component }\end{array}$ & \\
\hline
\end{tabular}

Table 1 Biomaterial Target Profile

*Specific targets adapted from (Zhou, Ma, Li, \& Yao, 2011), (Lee, Kasper, \& Mikos, 2014), and (Hing, Annaz, Saeed, Revell, \& Buckland, 2005).

**Based on the diffusion limit of oxygen in vivo of 100-200 $\mu$ m (Rouwkema, Rivron, \& van Blitterswijk, 2008).

***Adapted from (Turner, Wang, \& Burr, 2001) and dependent on direction with lower requirements for perpendicular forces.

\section{Summary}

Within the market the compromise between osteoconduction and strength has not been solved; the biomaterial target profile, in particular the sparse network macrostructure, has been developed to fill this gap. As such if the synthetic nacre can incorporate the porous structure and strength characteristics detailed then it is perceived to be of interest for further investment. 


\section{Proof of Concept}

\section{Overview}

When the synthetic nacre biomaterial has completed development and meets the target product profile a set of formal tests should be carried out before proceeding. The recommended order (based on technical risk, the critical path in material development, and cost) in which features or properties should be developed and tested begins with the porous structure and follows the flow chart below.

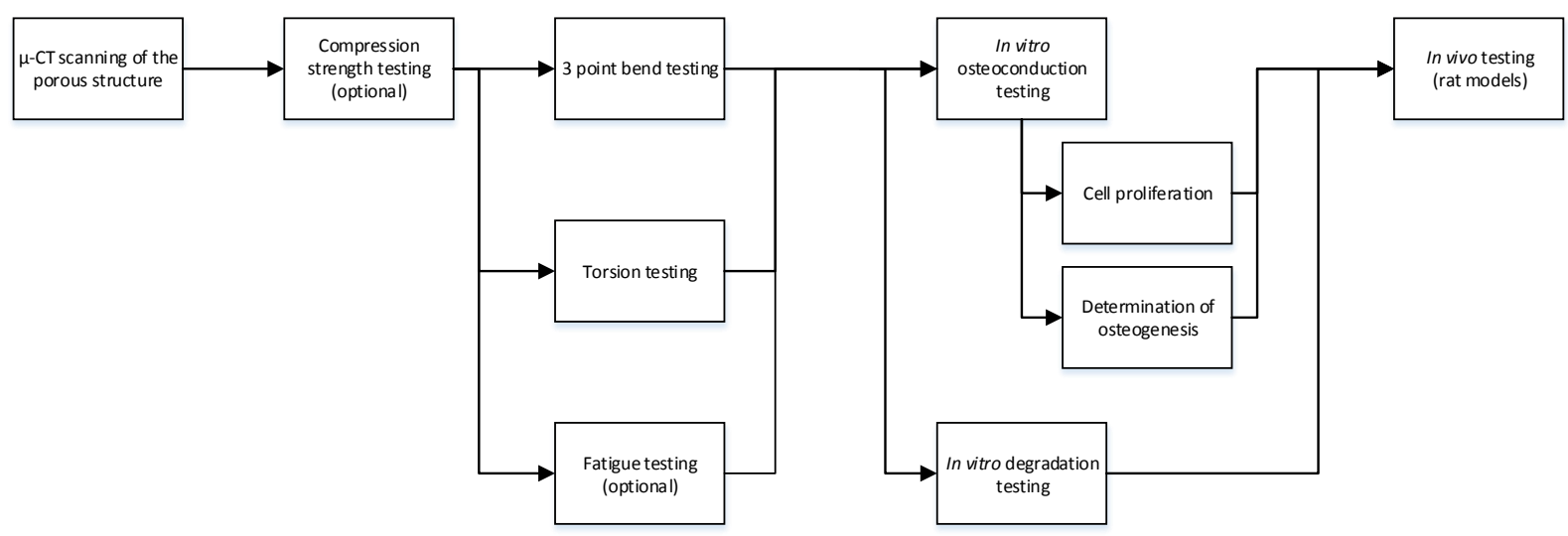

Figure 3 Proof of concept testing flow chart

\section{Mechanical}

The purpose of the mechanical testing is to determine whether the synthetic nacre biomaterial can incorporate a target porous structure, desirable to later achieve osteoconduction, and strength similar to natural cortical bone. Furthermore in vitro degradation testing will help determine whether it is able to maintain these desired strength properties for sufficient time, without debris, to enable complete healing in adults. Currently the material is without any porosity which is required both for the desired porous structure and to be able to manufacture specimens for all further testing.

\section{Porous $\mu$-structure}

Porosity with specific characteristics has been identified as a requirement before proceeding with strength or biological testing. There are several categories of pores mentioned in the material target profile but the presence of the macro level pores within the desired size range is the minimum requirement to support the proof of concept stage. 


\begin{tabular}{|llll|}
\hline \# & Feature & Target & Minimum \\
\hline $\mathbf{1}$ & Macro pores within range: & $200-300 \mu \mathrm{m}$ & $150-500 \mu \mathrm{m}$ \\
\hline $\mathbf{2}$ & Interconnecting tunnels & $60 \mu \mathrm{m}$ & $50-100 \mu \mathrm{m}$ \\
\hline $\mathbf{3}$ & Interconnectivity & $100 \%$ & $>70 \%$ \\
\hline
\end{tabular}

Table 2 Porous $\mu$-structure feature targets

There are various testing methods that can be utilised depending on availability and cost. During the development stage while implementing a porous structure SEM (Scanning Electronic Microscopy) and diffusion testing could be used to test for the presence, size, and interconnectivity of pores. For the final decision to continue to the next test $\mu$-CT scanning is suggested to get a precise model of the porous structure; this should return a result similar to Figure 4. ASTM F2450, Standard Guide for Assessing Microstructure of Polymeric Scaffolds for Use in Tissue Engineered Medical Products, may help provide information to build appropriate analysis procedures from a 3D scan.

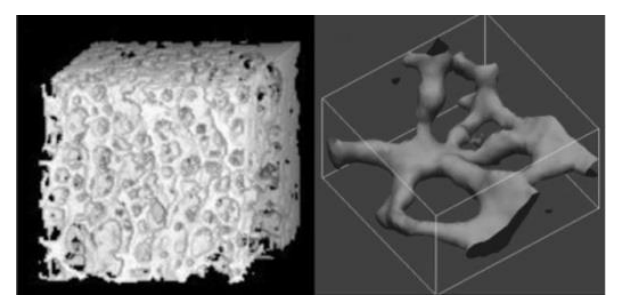

Figure 4 Image adapted from (Kherlopian, et al., 2008)

SEM/diffusion testing can be completed at VUW and there are $\mu$-CT scanner machines at the universities of Auckland and Otago (“NZ National Testing Facilities," 2014).

Cost: $\$ 200$ for a simple scan to $\$ 2000.00$ per scan for a large sample with analysis (Zamparo, 2011).

Strength

Research conducted into bone biomaterial market suggests that there is a gap for a biodegradable material, having good osteoconduction such that it requires a porous structure, with good mechanical properties. A positive strength in compression result is suggested before performing other tests. The end manufacturing process will include sterilisation via gamma irradiation - as this will cause cross-linking and make the material stronger it may not be necessary to include this process for these tests.

For each test:

- Use 10 specimens or more for statistical relevance.

- Test biomaterial at $37^{\circ} \mathrm{C}$ while the biomaterial is wet; if possible test in situ with specimen in soaking water or buffer solution. 
- Use the manufacturing of specimen shapes as a test that the 3D printer has the minimum capability requirements.

All tests can be completed in a universal testing machine in a temperature controlled cabinet; one of which is available at Callaghan Innovation or in various New Zealand universities other than VUW (“NZ National Testing Facilities," 2014). For the proof of concept a preliminary basic compression testing is optional while the 3 point bending and torsion tests, which will also provide a compression strength value, are required by regulations and should follow the relevant standards such as ASTM D7264 and F543. These two are important as torsion, or twisting, and bending are the two most common modes of failure for bone and implants (Väänänen, 2009). Additionally a standard fatigue test for biomaterials will need to be conducted either at this point or a later date such as after the initial biological testing.

Costs are in order of $\$ 20$ to $\$ 200$ per specimen with the exception of fatigue testing which is related to the number of cycles or duration.

\section{In vitro Degradation Testing}

Currently there are no standards for testing biodegradable biomaterials where the breakdown mechanism is enzymatic hydrolysis. This test has been adapted from other standards (ASTM F1635) and testing procedures suggested in literature, primarily (Azevedo \& Reis, 2005); as such this test can be adjusted with appropriate care.

Two profiles or rates are to be measured: the degradation, or remaining material; and strength, recording how the load bearing capabilities of the biomaterial change. This is essentially a repeated series of previously mentioned $\mu$-CT scans and standard based mechanical testing over a period of time of up to a minimum of 6 months. Allowing for early termination in the event the material has completely degraded the time point at which testing ends will depend on whether complete specimen degradation occurs in a reasonable fashion. The mechanical strength testing should be performed in situ where possible or immediately upon specimen removal from the buffer solution used to degrade the biomaterial; this should ensure the wet strength, not dry, is tested.

The buffer solution should mimic the internal environment of the human body that the final implant will be expected to be in. Due to synthetic nacre being a composite including a natural polymer component, chitin/chitosan, the standard phosphate buffer solution used by competing products to test degradation rates is not sufficient; the enzyme lysozyme needs to be included.

Additionally to mimic the blood flow and fluid dynamics within the body the container containing the enzyme and phosphate buffer solution need to be cycled two times per minute. 


\section{Biological}

The purpose of the biological testing is to determine whether osteogenic cells (bone cells) will grow on the biomaterial. The first stage is the in vitro testing: this involves determining whether the cells are capable of growing within the biomaterial and providing initial evidence support the statement that bone cells are capable of replacing the biomaterial with natural bone matrix. The second stage is the in vivo testing: this involves confirming that the biomaterial is degraded within the body and replaced with natural bone matrix; it also confirms a rate of degradation.

Currently there has been one test performed on the film form of the biomaterial. This test was performed using osteoblast cells which were incubated for 72 hours. The results showed that there was no immediate toxic effect to the osteoblast cells. The test did not demonstrate whether bone growth occurred due to the short incubation period. However, materials comprising chitin have been shown to support the growth of osteogenic cells and display osteoconductive features (US 13/801,044, 2013)(Di Martino, Sittinger, \& Risbud, 2005). As such the current assumption is that the biomaterial is capable of osteoconduction and is non-toxic to osteogenic cells.

\section{In vitro Testing}

The in vitro testing is estimated to take a period of 6 to 12 weeks to perform; the first aspect is to examine cellular proliferation of osteogenic cells. There are standard methods of determining cell proliferation such as AlamarBlue assays, PicoGreen dsDNA assays, or MTS assays. The AlamarBlue assay involves incubating the cells with a reagent which produces a colourmetric change (from blue to red) when incubated with live cells. The colourmetric change is measured using either the fluorescence or absorbance spectra wherein the percentage of colour change indicates cellular proliferation ("alamarBlue ${ }^{\circledR}$-Rapid \& Accurate Cell Health Indicator," n.d.). It would appear that the use of either AlamarBlue assays or PicoGreen assays are common tests utilised when determining osteoconductive capabilities of biomaterial scaffolds (Brey, Chung, Hankenson, Garino, \& Burdick, 2010; Fang, Wan, Tang, Gao, \& Dai, 2009; Musson et al., 2013; Subha N. Rath et al., 2012)

The next stage is to determine that the cells have adhered successfully to the biomaterial scaffold. This can be achieved using a combination of FDA/PI staining, fluorescent microscope, and scanning electron microscope. The FDA/PI is an assay which investigates the amount of live cells in a sample compared to dead cells. In particular, the cells are exposed to a compound called fluorescein diacetate and then propidium iodide. The viable cells are labelled green while the non-cells are labelled red. The biomaterial samples will then need to be examined under fluorescent microscope and scanning electron microscope. The scanning electron microscope can also show the proliferation 
of the cells throughout the entire 3D structure of the material(Subha N. Rath et al., 2012; Jones \& Boyde, 1977).

The last stage is to confirm that the biomaterial scaffold is osteoconductive and the cells begin the mineralisation process. Typical methods of determining whether osteogenic cells is by utilising reverse transcriptase polymerase chain reaction (RT-PCR) to ascertain the expression of genes associated with the regulation of osteogenesis (bone growth). The more common genes associated with osteogenesis that are measured are alkaline phosphatase, osteocalcin, osteopontin, RUNX-2 and collagen type I (Brey et al., 2010; Chen et al., 2012; Conserva, Foschi, Cancedda, \& Mastrogiacomo, 2013; Fang et al., 2009; Thibault, Scott Baggett, Mikos, \& Kasper, 2010).

\section{Beyond Proof of Concept}

A number of additional tests can or are required by regulation to be performed before the synthetic nacre biomaterial can gain pre-market approval. Following is an incomprehensive discussion of a number of future testing that may be undertaken and the various outcomes required.

\section{Fixation Testing}

Fixation is a known problem within orthopaedic implants and a potential advantage for the synthetic nacre material is the characteristic surface reaction that occurs in phosphate buffer solution. The calcium carbonate component of the biomaterial is dissolved and precipitated back as calcium phosphate (Ni, 2003), the same chemical composition as bone, and may form a chemical bond with nearby natural bone.

It is possible to test this and the fixation strength of the biomaterial before animal trials; this may be desirable to do so due to the lower cost and to build more medically relevant data and confidence around the future product. Fabricated bone screw specimens are inserted into natural bone, either cadaver or more available bovine bone, and placed in buffer solution over a number of days and the pull out strength measured at different time points. It may be possible to show that the surface reactions chemically bonds the implant to the surrounding bone holding the implant more firmly until osteoconduction occurs.

\section{Animal models}

The ISO 10993 standing for biocompatibility has a number of requirements for animal studies including to limit the number of and harm to the animals involved. Two different species are required and rats and sheep are suggested to combine toxicology, degradation, and mechanical testing within the same trial. 
Rats will be used for toxicology and testing that degradation is complete and osteoconduction occurs, as the biology of rats is very different from humans the rate at which degradation and osteoconduction occurs cannot be extrapolated.

Sheep are the second species as they, along with pigs, provide good models suitable for testing the repair of critical sized defects with load bearing implants (Peric et al., 2014). While pigs provide a closer model to humans sheep are suggested due to being more available and less expensive.

From the two trials, of which rats should be completed first, the biocompatibility testing should be completed as well as extrapolated profiles and effectiveness of the osteoconduction, degradation, and strength capabilities.

\section{Comparison to Controls}

To support market claims and for the purposes of filing patent documents appropriate controls need to be selected. In particular comparisons need to be made to existing products and the material described in US2014/0271914 to ensure healing times and efficacy is improved in the NacreTech biomaterial. 


\section{Manufacturing and Scale Up}

\section{Manufacturing and Material System}

Meeting the requirements for tailored material properties described in the material target product profile a concept has been described for a material and manufacturing system. Once developed, this system will use 3D printing, or potentially another high resolution rapid prototyping manufacturing method, to fabricate any number of different implant designs using tailored synthetic nacre biomaterial. Design trade-off curves for the different biomaterial features, such as porosity, strength, and degradation, will be developed allowing the synthetic nacre to be tailored as needed by implant designers or even surgeons directly. The combination will form a system that allows multiple implant product lines with a range of different material properties to be manufactured using the same equipment and processes - greatly simplifying and reducing the cost of production (Yildirimer \& Seifalian, 2014).

3D printing, particularly a variant similar to Fusion Deposition Modelling (FDM), was selected as the most viable method to obtain the adaptive requirements of the material as it has been shown in the lab by VUW researchers to work in concept. It currently works by printing the chitosan scaffold followed by the various chemical processes required to manipulate the material properties and perform the mineralisation of the calcium carbonate. 3D printing using FDM also has the benefit of future proofing the material and manufacturing by enabling developing technologies such as the inclusion of living cells, different materials, and drugs to be incorporated into the scaffold once they become available (Yildirimer \& Seifalian, 2014).

\section{Scale up of 3D Printing}

A well-known challenge is the manufacturing scale up of 3D printing (The Economist, 2013) therefore a basic model was developed to test the costs and production quantities associated with using it. One advantage the current process has is that unlike plastic FDM the chemical process for printing the chitosan requires no heating element; this could potentially ease the incorporation of multiple printing heads in the same machine to increase production rates.

In the final configuration the basic model (Figure 5, Page 47) was used to estimate that $203 \mathrm{D}$ printing machines could produce 25,000 bone screw units annually; the bone screws were estimated by $4 \mathrm{~mm}$ in diameter and $16 \mathrm{~mm}$ in length cylinders. Depending on the target breakeven point and units manufactured per year, 100,000 units over 4 years estimated here, this will produce cylinders at a little over $\$ 446$ each including overheads. 
Manufacturing Target Profile

\begin{tabular}{|c|c|c|}
\hline \# $\quad$ Feature & Target & Minimum \\
\hline \multicolumn{3}{|l|}{ 3D printing } \\
\hline Resolution & $\begin{array}{l}30 \mu m \text { or enabling control } \\
\text { over pore shape as well as } \\
\text { wall thickness }\end{array}$ & $\begin{array}{l}<200 \mu m \text { or the maximum } \\
\text { wall thickness in the porous } \\
\text { structure }\end{array}$ \\
\hline Deposition speed & $\begin{array}{l}>1000 \mu \mathrm{m} / \mathrm{s} \text { for higher } \\
\text { production rates }\end{array}$ & $>100 \mu \mathrm{m} / \mathrm{s}$ \\
\hline Printing heads & $\begin{array}{l}>10 \text { for higher product } \\
\text { rates }\end{array}$ & 1 \\
\hline Per printer capital cost & $<\$ 10,000$ & $<\$ 30,000$ \\
\hline Printer working life & $>1$ year & $>6$ months \\
\hline \multicolumn{3}{|l|}{ Sterilisation } \\
\hline Gamma irradiation & $\begin{array}{l}\text { In addition to sterilisation } \\
\text { used for crosslinking to } \\
\text { make the material } \\
\text { stronger }\end{array}$ & $\begin{array}{l}\text { Used for bulk sterilisation as } \\
\text { other methods, such as } \\
\text { autoclaving, are not } \\
\text { suitable for the biomaterial }\end{array}$ \\
\hline
\end{tabular}

Table 3 Manufacturing target profile

\section{Raw Material Limitation}

Natural polymers, including chitin/chitosan, have different characteristics depending on the source of the raw material and the process used during extraction and processing. With limited sources already a guaranteed supply from the same source needs to be acquired; since moving to a different source may change the properties (Aranaz et al., 2009) of the final material and/or may require changing the manufacturing process. 


\section{Intellectual Property Strategy}

\section{Patent Landscape}

There are three key aspects to the biomaterial which has been developed. First is the use of chitin as a scaffold which is mineralised with calcium carbonate (aragonite) to form the initial biomaterial. Second is the manufacturing methods utilised to produce a 3D biomaterial and incorporate the internal porous structure. Third is the use of the chitin and calcium phosphate combination in bone growth applications. These are the key areas which the intellectual property landscape is focused on and explored.

The use of chitin as a scaffold which is mineralised with calcium carbonate is well known in the art. The IPRP for the initial patent application cited Hosoda \& Kato (2001), US2004/0131562, Kato, Suzuki, \& Irie (2000), Zhang \& Gonsalves (1995), Wu, Cheng, Yao, Chen, \& Shao (2011) which all disclose chitin that has been crystallised with calcium carbonate. The scaffold structure of the chitin in these documents has been electrospun or is in film form. It is further important to note for future patents that the inventor's publications disclose the method of manufacturing the material and therefore any crystalline structure formation, such as aragonite (Munro \& McGrath, 2012), (Munro et al., 2013).

The methods disclosed above result in 2D forms of the chitin and calcium carbonate combination. There has been some investigation to incorporate a porous structure into a chitin scaffold. In summary, these methods are non solvent-solvent exchange (Pakavadee Ratanajiajaroen, \& Masahiro Ohshima, 2012), gas bubbling, freeze-drying (Yin et al., 2003), injection moulding (Fernandez \& Ingber, 2014) or porogens (Chevalier, Chulia, Pouget, \& Viana, 2008). These methods result in internal material structures that have the incorrect pore structure when compared to the current invention (biomaterial). New developments in the manufacture of chitin have been investigated in the area of 3D printing however the current resolution of that particular 3D printing method is not very high (Ang et al., 2002). There does not appear to be a known method of producing a chitin and calcium phosphate material which has a highly specific internal porous structure.

The use of the chitin and calcium carbonate combination in bone regrowth materials or bone scaffolds would appear to be novel at the time of this report. The most significant patent application to consider is US2014/0271914 which was filed by Orthovita on the 13 March 2013. The Orthovita patent teaches osteoconductive bone graft material which is made from the combination of calcium phosphate and chitin. It further teaches where the material has a micro-, meso- and macro-porous structure; however the method of manufacturing the pore structure is by freeze-drying. 
Additional patents have been filed by Matsumoto dental college, IKO KK, Genus EHF and Kyocera. These patents discuss the use of the chitin and calcium phosphate combination for use in bone regrowth/healing. However, the final material produced by these patents is sufficiently different (i.e. dough-like) and do not discuss a porosity within the material. These patents are of interest only and are not considered to significantly affect the patent strategy.

\section{Current State of Protection}

There is currently one application filed for the initial form of the biomaterial which was filed with PCT international (PCT application number PCT/IB2012/057197) with a priority date of the 12 December 2012. This application provides protection for the current method of manufacturing the biomaterial and for the biomaterial itself in film form. The international preliminary report on patentability (IPRP) and international search report (ISR) on this application has been issued and reports that the invention as claimed is novel and has an invention step. This PCT application has subsequently been entered in the United States of America for national phase.

\section{Intellectual Property Strategy}

The key aspects of the invention are: the composition of the biomaterial with the porous structure; the method of manufacturing the biomaterial; and the use of the biomaterial as a bone growth scaffold. It is noted that further development of the material and manufacturing process in currently being completed. As such the current patent application discussed above is not considered to provide full protection for the scope of the invention.

In view of the additional advances the next application will need to reflect the developments within the 3D structure, porosity, and strength of the biomaterial. The advantage of these characteristics is that they will allow for enhanced osteoconduction of the biomaterial which is considered to be one of the advantages our product will have over our competitors. In particular, the application will need to protect the new chemical method of manufacturing the material, the composition of the biomaterial, the porosity, and the 3D structure of the material.

The second aspect is regarding the use of the biomaterial; this will reflect the osteoconductive properties of the biomaterial and provide protection for the intended use as a bone growth scaffold. In order to file for the protection of the biomaterial for use as a bone growth scaffold. The team will need to have completed at least the in vivo based biological testing as the exemplification will provide support and enablement of the invention within the specification.

At this stage it is considered that the specific details regarding the method of $3 \mathrm{D}$ printing the material should remain trade secrets or will be protectable by patents. It is currently unclear what IP 
will result from the current development process. It is anticipated that much of the 3D printing will be based upon existing machinery and techniques but utilising them for a new material. Therefore, there is little benefit currently perceived in filing an additional patent to protect what may be minor variations to known methods. As such, these variations are considered to be best protected as trade secrets.

In regards to the US2014/0271914, the licensing of this patent will strengthen the patent portfolio of NacreTech add a biomaterial consisting of chitin and hydroxyapatite (calcium phosphate). The use of chitin and hydroxyapatite is a logical next step in the development of the biomaterial and therefore licensing the patent will benefit NacreTech. The license of the patent will also protect us from any infringement suit from Stryker.

\section{Filing Dates and Locations}

The date of filing the patent applications is considered vital as the 20 year limitation will be consumed primarily by gaining regulatory approval. However, prolonging the filing date increases the risk of another patent application being filed which anticipates or prevents the acceptance of NacreTech's invention. Therefore, in view of the regulatory considerations and the requirements under support and enablement it is proposed that the best window for filing the patent applications will be shortly before the completion of the in vivo based biological testing but before the preclinical testing.

In addition, it is proposed that an initial provisional application is filed through the New Zealand patents office. This will then allow for 12 months to complete all in vivo biological testing before filing a complete PCT specification at WIPO in view of the Paris convention. The specification will contain all the details regarding the chemical manufacture of the material and the use of the biomaterial as a bone growth scaffold. If it is determined that the application cannot be prosecuted in a single application due to unity, then the applications can be divided out into the two areas for protection. This method of filing is considered to reduce PCT and ISR costs, while ensuring there is sufficient support and enablement for both areas for protection.

The primary filing countries are considered to be the United States of America and the European Union, as these are our largest target markets. This is followed by the countries of manufacture such as India, Mexico and China. Lastly we will be filing in the second tier countries such as Australia, New Zealand, Canada, Japan and South Korea. 


\section{Regulatory Considerations}

\section{Definition of a Medical Device}

A medical device is defined as an article which is intended by the manufacturer to be used for human beings for the purpose of treatment of an injury, disease or modification of the anatomy or physical function, wherein the principal intended action in the human body is not achieved by pharmacological or metabolic means but which may be assisted by such means (as per Medical Device Directive, MDD (93/42/EEC)).

The bioactive bone screw is considered to be a medical device as the intended purpose of the device is to act as a screw for the purpose of treating an injury. The bioactive feature of the screw is not considered to be the principal intended action but assists the intended purpose of the device. Therefore, as the bone screw is a medical device we are required to obtain regulatory approval for the device before the device can be sold.

\section{Explanation of Regulatory Systems}

There are two primary systems in order to obtain regulatory approval. The first is the FDA which grants regulatory approval for the United States of America, while the other is CE Mark which grants regulatory approval for the European Union. Each of these systems have slight differences which affect the speed, cost and risk associated with obtaining regulatory approval and will be discussed further below

The FDA has two main routes to market for medical devices. The first is via a Premarket Notification (510k) which is based upon the manufacturer providing satisfactory evidence that a device is substantially equivalent to an existing device with regulatory approval. The second is via a Premarket Approval (PMA) which requires clinical data to support the safety and efficacy of the device.

The CE mark regulations for medical devices is disclosed in the Medical Device Directive, MDD (93/42/EEC). In order to obtain regulatory approval the application needs to meet conformity assessment procedure as set out in the Annexes of the MDD (93/42/EEC), however which annexes a device needs to meet is determined by their classification. The classifications (Is, Im, Ila, IIb and III) define a medical device determine the relative risk of the device dependent upon how long the device is intended to be in continuous use, whether the device is invasive, whether the device is implantable or active, and whether the device contains a substance which is a medicinal substance or has an ancillary function to the device. Hence, the more invasive or the longer a medical device is in the body, the higher the risk and therefore the higher the classification number. 
There are several key differences between the FDA and CE Mark systems. The first is the aspects of the clinical data which are examined during application. In particular the CE mark examines primarily on the safety of the medical device while the FDA examines on the safety and efficacy of medical device. Hence, to obtain regulatory approval through the FDA the application needs to show that the device is both safe to a humans but also fulfils the claimed function. The effect of the differences in the examination of the application, is the CE mark system is often faster to grant regulatory approval than the FDA. It is further noted that the FDA will accept foreign clinical data in support of an application, however there are specific formalities as per the IDE in order for the data to be allowable. In particular, clinical studies for CE mark need to fulfil the Helsinki accords, however the FDA requires meeting Sec. 812 of Subchapter $\mathrm{H}$ of the Code of Federal Regulations, Food and Drugs.

\section{Key Considerations}

To gain regulatory approval a pre-clinical investigation needs to be conducted into the biocompatibility of the biomaterial in the form of a bioactive bone screw. The pre-clinical investigation needs to be conducted in line with ISO 10-993. ISO 10-993 sets out the requirements for testing genotoxicity, carcinogenicity, reproductive toxicity, in vitro cytoxicity, sensitization, and biodegradation testing. These tests are required before filing for an IDE exemption from the FDA to perform clinical trials; it is noted that many of the tests detailed in the ISO 10-993 can be carried out during animal trials in the proof of concept stage.

At the time of writing this report no medical device or drug has been granted regulatory approval utilising chitin within the human body. As a result the bioactive bone screw is considered to be a Class III medical device in both regulatory systems. Therefore clinical trials will need to be performed in accordance with both the Helsinki accords and Sec 812 of the FDA act. Performing the clinical trials will increase the cost of development and time to market significantly which is estimated between $\$ 10-15$ million and six to eight years. The benefit to performing clinical trials is that it increases clinician trust in the product and the brand; existing biomaterial bone screws have been granted regulatory approval based on the $510 \mathrm{k}$ process and therefore do not have any substantial clinical data before market approval.

To ensure that the submissions are in accordance with all relevant sections and appendices of the FDA and Medical Device Directive an important consideration will be to find regulatory specialists which are familiar with biomaterials and medical devices. Further areas which the will need to be considered upon completion of additional development is adherence to the goods manufacturing process, sterilisation of the device, and packaging. 


\section{Financial Overview}

The financial information regarding the separate sections has been listed and summed here to provide an estimate of the total cost to market. The production and cost model of the synthetic nacre material and 3D printer based manufacturing has been included, configured for bone screws, and its result justified with respect to expected cost compared to other products. The values are believed to be conservative at the time of writing but should not be considered accurate; for example much of the later, more expensive, costs would be conducted overseas and is subject to currency exchange rates.

\section{Commercialisation Costs}

Following is a summary of a number of costs involved in the bone project up until, and including an estimate of, the clinical trials stage.

\section{Task}

Cost(US\$়)

Material and 3D printer development

(seed funding to be sourced by research team)

$500,000.00$

Proof of Concept testing (Series A)

\begin{tabular}{|lr|}
\hline $\boldsymbol{\mu}$-CT scanning & $2,000.00$ \\
\hline Compression strength testing & $2,000.00$ \\
\hline 3-point bending testing & $2,000.00$ \\
\hline Torsion testing & $2,000.00$ \\
\hline Fatigue testing* & $100,000.00$ \\
\hline In vitro degradation testing & $100,000.00$ \\
\hline In vitro biological testing & $200,000.00$ \\
\hline Pre-clinical trials** & $\mathbf{2}$ \\
\hline
\end{tabular}

Intellectual Property Protection

Patents***

Total (Series A)

$608,000.00$

*Figures not available; determined by duration.

**Initial estimation through consultation with regulatory specialist.

***Takes into consideration attorney fees, filing fees, and maintenance costs over the life of the patents.

\section{Series B}

FDA approval costs including clinical trials

(Makower, Meer, \& Denend, 2010)

$75,000,000.00$

Overall total (Series A + B)

$75,608,000.00$

Table 4 Cost of commercialisation summary 


\section{D Printing Production and Cost Model}

Below is a model of the 3D printer screw production, represented by cylinders, and the final cost to manufacture each screw. The final result ( $\$ 446$ per screw), this is considered to be an acceptable cost per screw in view of the proposed health benefits outlined in the value proposition (p. 48). This highlights the importance of achieving significantly improved efficacy over current products and/or reduced total cost of care - synthetic nacre implants, using 3D printing, will be significantly more expensive. However this model is based off only one implant, whereas the adaptive manufacturing system should allow expanding to a range a products (spreading the cost to market) across greater production.

\begin{tabular}{|c|c|c|c|c|c|c|}
\hline \multicolumn{4}{|c|}{ Raw materials } & \multicolumn{3}{|c|}{ Cost to market } \\
\hline Cylinder & & Porosity & Density $\left(\mathrm{Mg} / \mathrm{m}^{3}\right)$ & Stages & & Total \\
\hline Diameter (mm) & 4 & $60 \%$ & 3 & Phase A & $608,000.00$ & $75,608,000.00$ \\
\hline \multirow[t]{2}{*}{ Length $(\mathrm{mm})$} & 16 & & Density $\left(\mathrm{g} / \mathrm{mm}^{3}\right)$ & Phase B & $75,000,000.00$ & \\
\hline & & & 0.003 & & & \\
\hline pi & 3.141592654 & & Cost/gram & & & \\
\hline r & 2 & & \begin{tabular}{|r|}
$\$ 2.10$ \\
\end{tabular} & & & \\
\hline \multirow[t]{2}{*}{$\mathrm{h}$} & 16 & & & \multicolumn{3}{|c|}{ Cost-driven price } \\
\hline & & & & Breakeven (units) & & Total cost/cylinder \\
\hline Volume $\left(\mathrm{mm}^{3}\right)$ & 201.0619298 & 120.6371579 & & \begin{tabular}{|r|}
100000 \\
\end{tabular} & & \begin{tabular}{|r|}
446.8325555 \\
\end{tabular} \\
\hline Mass (g) & 0.603185789 & 0.361911474 & & & \multicolumn{2}{|c|}{ \# machines for 4 year breakeven } \\
\hline \multirow[t]{3}{*}{ Raw materials } & $\$ 1.27$ & $\$ 0.76$ & & Overheads & & \begin{tabular}{r|}
21 \\
\end{tabular} \\
\hline & & & & Operations & Marketing & with annual units of \\
\hline & & & & $30 \%$ & $20 \%$ & 25869 \\
\hline \multicolumn{4}{|c|}{ 3D printing } & \multicolumn{3}{|l|}{ Production } \\
\hline Speed $(\mathrm{mm} / \mathrm{s})$ & \multicolumn{2}{|c|}{ Extrusion diameter (mm) } & & Heads per printer & 3D printer cost & Printer Life (months) \\
\hline 1 & \multicolumn{2}{|c|}{0.05} & & \begin{tabular}{|r|} 
\\
4
\end{tabular} & $\$ \quad 10,000.00$ & 6 \\
\hline $\mathrm{V} / \mathrm{s}\left(\mathrm{mm}^{3} / \mathrm{s}\right)$ & Total time fo & or cylinder (s) & Hours & 30 days (cylinders) & 365 days (cylinders) & Printer cost/cylinder \\
\hline \multirow[t]{2}{*}{0.001963495} & 0\% Porosity & 102400 & 28.44 & 101.25 & $\begin{array}{r}1231.88 \\
\end{array}$ & $\$ \quad 16.46$ \\
\hline & $60 \%$ Porosity & 61440 & 17.07 & 168.75 & 2053.13 & 9.88 \\
\hline Electricity & & & & \multicolumn{2}{|c|}{ Additional costs/screw } & \\
\hline Current (A) & 6 & Units/Printer & & \multicolumn{2}{|c|}{ cGMP Contract Manufacturing } & 20.00 \\
\hline Voltage (V) & 24 & 30 Days & & \multicolumn{2}{|c|}{ Post-processing } & 30.00 \\
\hline Power (W) & 144 & 365 Days & & & & \\
\hline kW.h/cylinder & 4.096 & & & & & \\
\hline Cost/kW.h & 0.26 & & & \multicolumn{2}{|c|}{ Production cost/screw } & \\
\hline Cost/cylinder & 1.06 & & & $\$$ & 68.79 & \\
\hline
\end{tabular}

Figure 5 3D production and cost model - This model is incomplete and makes some assumptions about the 3D printing method used, such as extrusion diameter, which may be incorrect.

\section{Summary}

At this early stage without knowing exactly what is required in terms of regulatory processes and material development and testing the total cost to reach market is an estimate. It is acknowledged that the cost to market of a single application is large therefore a product manufacturing platform strategy will expand the product range and as a result reduce the associated risks. 


\section{Value Proposition Summary}

\section{Material Advantages}

One of the features of the material is the formation of aragonite crystal. The aragonite, a form of calcium carbonate, is known to be stronger than the more common calcite (Roberts, 2009). Existing bone substitutes on the market comprising calcium carbonate such as coral only possess the calcite formation; as such a perceived advantage of the synthetic nacre material is increased strength.

The other composite constitute is the natural polymer chitin/chitosan which while retaining the strength of synthetic polymers breaks down into non-acidic by-products (Azevedo \& Reis, 2005). This is a perceived advantage of the existing PLA based materials currently used in the market.

The adaptive manufacturing concept includes a bottom up fabrication process, using 3D printing, which may simplify the production of multiple implant designs and types. This is a perceived advantage over existing methods for producing biomaterial implant products as it eliminates the need for multiple complex manufacturing steps required for different designs.

An additional perceived advantage of the material and manufacturing system is the ability to tailor the synthetic nacre biomaterial properties and product design to individual patient or surgeons requirements. This personalised medicine capability is currently not known to be available in any orthopaedic biomaterial.

\section{Healthcare Economics}

Significant benefits are available through the uptake of biodegradable bone screws. The main beneficiaries are expected to be both health care providers such as hospitals and insurance companies as the intention is to reduce the overall cost of treatment. Medical devices for the initial surgery are expected to increase in cost but these are believed to be offset by removing the need for additional surgery to removed said device.

Without the need for a second surgery it is expected that overall there will be additional resources available which can be used elsewhere. Saving in other relevant areas are expected, for example faster recovery timelines will result in reduced bed loads within the hospital. The knock-on effects of this are more effective use of hospital resources such as surgeon time as well as a reduction of waiting lists as the total number of surgeries will have been reduced. 


\section{Value to Patients}

Patients would benefit from the reduction of metallic devices both during the recovery process and that which remains once healing is complete. The other significant benefit for patients is from removing the need for a second surgery as this will support both their physical needs and reduce the psychological impact. It is expected that the length of time spent in hospital will be reduced, hospital stays can sometimes be harmful or even fatal for patients (Ulrich, Quan, Zimring, Joseph, \& Choudhary, 2004). It is expected that healing rates will be increased enabling faster overall recovery because of the gradual transfer of stress to the healing bone, better overall patient outcomes (Hovis, Kaiser, Watson, \& Bucholz, 2002). 


\section{Future Project Development}

\section{Market Research}

The material development is expected to take a significant amount of time, once development is complete it is recommended that the market research is repeated and taken to the next level of accuracy. To hasten the process it is recommended that a global report is purchased.

\section{Application Development Team}

Once the material is developed to such a stage that it become possible to know what can or cannot be made with the material it is recommended that an application development and design team is created. This team is intended to work with a number of potential users to find the initial specific surgical application for NacreTech to target.

\section{Technical Development}

It is necessary for the biomaterial to be developed to include at least the porosity and strength before further testing or development stages is started. Particularly interconnected porosity is required if there is any chance of achieving high quality osteoconduction. If the biomaterial is unable to achieve the porosity and strength targets outlined in the TPP then the target application as an orthopaedic biomaterial would not appear to be viable.

\section{TPP Individual Implant Requirements and Testing}

The current target profiles are for the biomaterial in the general load bearing use case and has not been developed for specific use cases as the current strength characteristics of the synthetic nacre material have not been determined. Bone screws is a subset of orthopaedic implants and individual applications will have their own variations. Once the first surgical procedure and its corresponding implant requirements are identified the TPP will need to be modified to reflect the specific requirements of the implant.

\section{Plant Automation and Costs}

The process regarding the $3 \mathrm{D}$ printing of the biomaterial is still underdevelopment, as a result the financial and scale-up model is only an estimate. Once the process is finalised, this model will need to be re-addressed. In addition, the cGMP manufacturing figure currently includes an estimate of the automation steps for handling, for example packaging and labelling which may not accurately reflect the final value given changes to the manufacturing processes. 


\section{Further Intellectual Property}

The proposed intellectual property strategy above considers only the intellectual property which has been developed or is currently being developed within Victoria University. However, there are additional areas to which IP will need to be created or licensed in order to commercialise the biomaterial and market a bioactive bone screw. The following areas are detailed below

\section{Internal Pore Structure}

The internal pore structure is the precise network of interconnected pores which allow for the osteoconduction but also optimise the strength of the material. Possibly IP could be sourced from ... (person who created the 3D model of a bones interior) or through the Victoria design school.

\section{Medical Device Design}

The design of the device is dependent upon the material strength results from the first stage of biomaterial testing. The strength of the material will provide guidance on specific uses within orthopaedic surgery and therefore which screw design will be needed. Further investigation is required within this area.

\section{D Printing}

As the 3D printing process is still under development, it is possible that licensing agreements may be required.

\section{Regulatory}

It is advised that a regulatory expert is acquired early into the proof of concept stage to ensure that the testing meets regulatory standards and to develop the specific plan to gain regulatory acceptance. Regulatory standards have been considered within the proof of concept plan, however only at a high level planning stage. 


\section{Bibliography}

Adams, R. (2010). If you build it will they come?: three steps to test and validate any market opportunity. Hoboken, N.J: Wiley.

Ahlers, G. K. C., Cumming, D. J., Guenther, C., \& Schweizer, D. (2012, October 14). Signaling in Equity Crowdfunding. Retrieved February 26, 2015, from http://dx.doi.org/10.2139/ssrn.2161587 alamarBlue ${ }^{\circledR}$-Rapid \& Accurate Cell Health Indicator. (n.d.). Retrieved February 21, 2015, from http://www.lifetechnologies.com/nz/en/home/brands/molecular-probes/key-molecularprobes-products/alamarblue-rapid-and-accurate-cell-health-indicator.html

Ang, T. ., Sultana, F. S. ., Hutmacher, D. ., Wong, Y. ., Fuh, J. Y. ., Mo, X. ., ... Teoh, S. . (2002). Fabrication of 3D chitosan-hydroxyapatite scaffolds using a robotic dispensing system. Materials Science and Engineering: C, 20(1-2), 35-42.

Anonymous. (2014). Bioretec Ltd. Equity Issue 2014 (pp. 1-15).

Aranaz, I., Mengíbar, M., Harris, R., Paños, I., Miralles, B., Acosta, N., ... Heras, Á. (2009). Functional characterization of chitin and chitosan. Current Chemical Biology, 3(2), 203-230.

Ashkenas, R. $(2012,05)$. It's Time to Rethink Continuous Improvement [Harvard Business Review]. Retrieved from https://hbr.org/2012/05/its-time-to-rethink-continuous/

Azevedo, H. S., \& Reis, R. L. (2005). Understanding the enzymatic degradation of biodegradable polymers and strategies to control their degradation rate. Biodegradable Systems in Tissue Engineering and Regenerative Medicine. Boca Raton, FL: CRC Press, 177-201.

Belleflamme, P., Lambert, T., \& Schwienbacher, A. (2013). Crowdfunding: Tapping the right crowd. Journal of Business Venturing. Retrieved from http://dx.doi.org/10.1016/j.jbusvent.2013.07.003

Böstman, O., \& Pihlajamäki, H. (2000). Clinical biocompatibility of biodegradable orthopaedic implants for internal fixation: a review. Biomaterials, 21(24), 2615-2621.

Boyce, L. (2013, June 26). BrewDog "Equity of Punks" crowdfunding proves an instant hit by raising f1m in a day - but is it a serious investment? [Newspaper]. Retrieved from 
http://www.thisismoney.co.uk/money/investing/article-2348203/BrewDog-Equity-Punkscrowdfunding-proves-instant-hit-drinkers--investment.html

Brey, D. M., Chung, C., Hankenson, K. D., Garino, J. P., \& Burdick, J. A. (2010). Identification of Osteoconductive and Biodegradable Polymers from a Combinatorial Polymer Library. Journal of Biomedical Materials Research. Part a, 93(2), 807-816.

Brinded, L. (2014). Scotland's Brewdog Defies Industry Norms and Applies Living Wage Policy for UK Staff. Retrieved from http://www.ibtimes.co.uk/scotlands-brewdog-defies-industry-normsapplies-living-wage-policy-all-staff-1468351

Bryman, A., \& Bell, E. (2007). Business Research Methods (Second). Oxford: Oxford university press.

Chen, Y., Huang, Z., Li, X., Li, S., Zhou, Z., Zhang, Y., ... Yu, B. (2012). In Vitro Biocompatibility and Osteoblast Differentiation of an Injectable Chitosan/Nano-Hydroxyapatite/Collagen Scaffold. Journal of Nanomaterials, 2012, e401084. doi:10.1155/2012/401084

Chevalier, E., Chulia, D., Pouget, C., \& Viana, M. (2008). Fabrication of porous substrates: A review of processes using pore forming agents in the biomaterial field. Journal of Pharmaceutical Sciences, 97(3), 1135-1154.

ConMed Corporation. (2014, 02). CONMED Corporation Announces Board and Governance Changes. Retrieved from http://www.marketwired.com/press-release/conmed-corporationannounces-board-and-governance-changes-nasdaq-cnmd-1882814.htm

Conserva, E., Foschi, F., Cancedda, R., \& Mastrogiacomo, M. (2013). In vitro and in vivo osteoinductive and osteoconductive properties of a synthetic bone substitute. The International Journal of Oral \& Maxillofacial Implants, 28(6), e432-439.

Di Martino, A., Sittinger, M., \& Risbud, M. V. (2005). Chitosan: A versatile biopolymer for orthopaedic tissue-engineering. Biomaterials, 26(30), 5983-5990.

Fang, B., Wan, Y.-Z., Tang, T.-T., Gao, C., \& Dai, K.-R. (2009). Proliferation and Osteoblastic Differentiation of Human Bone Marrow Stromal Cells on Hydroxyapatite/Bacterial Cellulose 
Nanocomposite Scaffolds. Tissue Engineering Part A, 15(5), 1091-8.

doi:http://dx.doi.org.helicon.vuw.ac.nz/10.1089/ten.tea.2008.0110

Fernandez, J. G., \& Ingber, D. E. (2014). Manufacturing of Large-Scale Functional Objects Using Biodegradable Chitosan Bioplastic. Macromolecular Materials and Engineering, 299(8), 932938.

Forbes.com. (2014, October). Biomet. Retrieved from http://www.forbes.com/companies/biomet/ Gefen, A. (2002). Optimizing the Biomechanical compatibility of orthopedic screws for bone fracture fixation. Medical Engineering \& Physics, 24, 337-347.

Gleckman, H. $(2014,11)$. How Did Medical Device Makers Become Poster Children for Obamacare Critics? [Newspaper]. Retrieved February 25, 2015, from http://www.forbes.com/sites/beltway/2014/11/13/how-did-medical-device-makersbecome-poster-children-for-obamacare-critics/

Gower, L. B., Olszta, M. J., Douglas, E. P., Munisamy, S., \& Wheeler, D. L. (n.d.). Fluid swellable fibrous matrix containing minerals; artificial bone.

Hanson, B., van der Werken, C., \& Stengel, D. (2008). Surgeons' beliefs and perceptions about removal of orthopaedic implants. BMC Musculoskeletal Disorders, 9, 73-73. doi:10.1186/1471-2474-9-73

Hing, K. A., Annaz, B., Saeed, S., Revell, P. A., \& Buckland, T. (2005). Microporosity enhances bioactivity of synthetic bone graft substitutes. Journal of Materials Science: Materials in Medicine, 16(5), 467-475.

Holmes, K. R. (2011). Thermal properties. Cortex (dog), 491, 16.

Hosoda, N., \& Kato, T. (2001). Thin-Film Formation of Calcium Carbonate Crystals: Effects of Functional Groups of Matrix Polymers. Chemistry of Materials, 13(2), 688-693.

Hovis, W. D., Kaiser, B. W., Watson, J. T., \& Bucholz, R. W. (2002). Treatment of Syndesmotic Disruptions of the Ankle with Bioabsorbable Screw Fixation. The Journal of Bone \& Joint Surgery, 84(1), 26-31. 
Industry Experts. (2011). Biomaterials - A global market overview.

Johnsson, T., \& Hagg, I. (1987). Extrapreneurs-Between Markets and Hierarchies. International Studies of Management \& Organization, 17(1), 64-74.

Jones, S. J., \& Boyde, A. (1977). The migration of osteoblasts. Cell and Tissue Research, 184(2), 17993.

Joukainen, A. (2008). New Bioabsorbable Implants for the Fixation of Metaphyseal Bone. Kuopio University.

Kato, T., Suzuki, T., \& Irie, T. (2000). Layered Thin-Film Composite Consisting of Polymers and Calcium Carbonate: A Novel Organic/Inorganic Material with an Organized Structure. Chemistry Letters, (2), 186-187.

Klein, G. (2013). Seeing what others don't: The remarkable ways we discover insights.

Kousa, P., Jarvinen, T. L., Pohjonen, T., Kannus, P., Kotikoski, M., \& Jarvinen, M. (1995). Fixation strength of a biodegradable screw in anterior cruciate ligament reconstruction. Journal of Bone \& Joint Surgery, British Volume, 77(6), 901-905.

Lee, E. J., Kasper, F. K., \& Mikos, A. G. (2014). Biomaterials for Tissue Engineering. Annals of Biomedical Engineering, 42(2), 323-337.

Makower, J., Meer, A., \& Denend, L. (2010). FDA impact on U.S. Medical Technology Innovation. Retrieved from http://www.google.co.nz/url?sa=t\&rct=j\&q=\&esrc=s\&source=web\&cd=1\&ved=0CB4QFjAA\& url=http\%3A\%2F\%2Feucomed.org\%2Fuploads\%2FPress\%2520Releases\%2FFDA\%2520impact \%2520on\%2520U.S.\%2520Medical\%2520Technology\%2520Innovation.pdf\&ei=vbsVIfmEcT4mAWatYGgDQ\&usg=AFQjCNHNSLExeOHJwxehXqEkNpkeAJBhOQ\&bvm=bv. 86475 890, d.dGY\&cad=rja

Markets.ft.com. (2015, February 25). Takiron Co Lts. Retrieved February 25, 2015, from http://markets.ft.com/research/Markets/Tearsheets/Business-profile?s=4215:TYO 
Markides Constantinos, \& Sosa, L. (2013). Pioneering and First Mover Advantages: The Importance of Business Models. Long Range Planning, 46(4-5), 325-334.

medicaldevicedevelopments. (n.d.). Invibio Biomaterial Solutions - A step ahead.

medicaldevicedevelopments. Retrieved from http://www.medicaldevice-

developments.com/contractors/biomaterials-and-biotechnology/invibio-biomaterialsolutions/

MedTech Europe. (2012). The European Medical Technology Industry in Figures. The European Medical Technology Industry in Figures.

Moncrieff, J. (1999). Is strategy making a difference? Long Range Planning, 32(2), 273-276. doi:10.1016/S0024-6301(99)00033-3

Morrison, A., \& Wensley, R. (1991). Taylor \& Francis Online :: Boxing up or Boxed in?: A Short History of the Boston Consulting Group Share/ Growth Matrix - Journal of Marketing Management Volume 7, Issue 2. Journal of Marketing Management, 7(2), 105-129. doi:10.1080/0267257X.1991.9964145

Munro, N. H., Green, D. W., \& McGrath, K. M. (2013). In situ continuous growth formation of synthetic biominerals. Chemical Communications, 49(33), 3407-3409.

Munro, N. H., \& McGrath, K. M. (2012). Biomimetic approach to forming chitin/aragonite composites. Chemical Communications, 48(39), 4716-4718.

Musson, D. S., McIntosh, J., Callon, K. E., Chhana, A., Dunbar, P. R., Naot, D., \& Cornish, J. (2013). The Need for Thorough in Vitro Testing of Biomaterial Scaffolds: Two Case Studies. Procedia Engineering, 59, 138-143. doi:10.1016/j.proeng.2013.05.103

Nielsen. (2015, January 20). Growing Global Health Awareness Could Mean Big Business For Manufacturers. Retrieved February 28, 2015, from http://www.prnewswire.com/newsreleases/growing-global-health-awareness-could-mean-big-business-for-manufacturers300023319.html 
$\mathrm{Ni}, \mathrm{M}$. (2003). Nacre surface transformation to hydroxyapatite in a phosphate buffer solution. Biomaterials, 24(23), 4323-4331.

NZ National Testing Facilities. (2014, September 20). NZProduct Accelerator. Retrieved from http://www.materialsaccelerator.co.nz/nationaltestings.html

O'leary, Z. (2010). The essential guide to doing your research project. Thousand Oaks: Sage Publications.

Or, C. K. L., \& Karsh, B.-T. (2009). A Systematic Review of Patient Acceptance of Consumer Health Information Technology. Journal of the American Medical Informatics Association, 16(4), 550-560. doi:10.1197/jamia.M2888

Orthoworld. (2010). The Orthopaedic industry annual report : 2009-2010 (pp. 1-180).

Pakavadee Ratanajiajaroen, \& Masahiro Ohshima. (2012). Preparation of highly porous-chitin structure through nonsolvent-solvent exchange-induced phase separation and supercritical CO2 drying. The Journal of Supercritical Fluids, 68, 31-38.

Peng, M. W. (2012). The global strategy of emerging multinationals from China. Global Strategy Journal, 2, 97-107. doi:10.1111/j.2042-5805.2012.01030.x

Peric, M., Dumic-Cule, I., Grcevic, D., Matijasic, M., Verbanac, D., Paul, R., ... Vukicevic, S. (2014). The rational use of animal models in the evaluation of novel bone regenerative therapies. Bone. Retrieved from http://linkinghub.elsevier.com/retrieve/pii/S8756328214002622

Pilia, M., Guda, T., \& Appleford, M. (2013). Development of Composite Scaffolds for Load-Bearing Segmental Bone Defects. BioMed Research International, 2013, 1-15. doi:10.1155/2013/458253

Raine, J., O'Reilly, P., Teicher, M., New Zealand, \& Ministry of Science and Innovation. (2011). Powering innovation: improving access to and uptake of $R \& D$ in the high value manufacturing and services sector. Retrieved from http://www.msi.govt.nz/assets/1.pdf Roberts, J. M. (Ed.). (2009). Cold-water corals: the biology and geology of deep-sea coral habitats. Cambridge, UK ; New York: Cambridge University Press. 
Robinson, D. T., \& Stuart, T. E. (2006). Network Effects in the Governance of Strategic Alliances. Journal of Law, Economics, and Organization, 23(1), 242-273. doi:10.1093/jleo/ewm010

Rouwkema, J., Rivron, N. C., \& van Blitterswijk, C. A. (2008). Vascularization in tissue engineering. Trends in Biotechnology, 26(8), 434-441.

Schaper, M., Volery, T., Weber, P., \& Lewis, K. (2011). Entrepreneurship and small business (3rd AsiaPacific ed). Milton, Qld: John Wiley \& Sons Australia.

Solomon, M. R. (2008). Marketing: real people, real choices. Oamaru, N.Z.: Pearson Education New Zealand.

Subha N. Rath, Leonie A. Strobel, Andreas Arkudas, Justus P. Beier, Anne-Kathrin Maier, Peter Greil, ... Ulrich Kneser. (2012). Osteoinduction and survival of osteoblasts and bone-marro stromal cells in 3D biphasic calcium phosphate scaffolds under static and dynamic culture conditions. J. Cell. Mol. Med., 16(10), 2350-2361.

Suchman, M. C. (2012). Managing Legitimacy: Strategic and Institutional Approaches. The Academy of Management Review, 20(3), 571-610.

Summers, L. H. (1989). Some simple economics of mandated benefits. The American Economic Review, 79(2), 177-183.

Teoh, S. H. (2000). Fatigue of biomaterials: a review. International Journal of Fatigue, 22(10), 825837.

The Economist. (2013, September 7). 3D printing scales up. The Economist, 408(8852), 11.

Thibault, R. A., Scott Baggett, L., Mikos, A. G., \& Kasper, F. K. (2010). Osteogenic Differentiation of Mesenchymal Stem Cells on Pregenerated Extracellular Matrix Scaffolds in the Absence of Osteogenic Cell Culture Supplements. Tissue Engineering Part A, 16(2), 431-40. doi:http://dx.doi.org.helicon.vuw.ac.nz/10.1089/ten.tea.2009.0583

Torr-Brown, S. (2013). Crowdsourcing for Science and Medicine: Progress and Challenges. The Journal of OncoPathology, 1(2), 75-81. 
Transparency Market Research. (2013). Trauma Fixation Devices Market Global Industry Analysis, Growth,Trends and Forecast 2019. Retrieved from http://www.transparencymarketresearch.com/trauma-fixation-devices-market.html

Truumees, E., \& Herkowitz, H. N. (1999). Alternatives to Autologous Bone Harvest in Spine Surgery. The University of Pennsylvania Orthopaedic Journal, 12, 77-88.

Turner, C. H., Wang, T., \& Burr, D. B. (2001). Shear Strength and Fatigue Properties of Human Cortical Bone Determined from Pure Shear Tests. Calcified Tissue International, 69(6), 373378.

Ulrich, R., Quan, X., Zimring, C., Joseph, A., \& Choudhary, R. (2004). The role of the physical environment in the hospital of the 21 st century: a once-in-a-lifetime opportunity (pp. 1-69). Retrieved from http://static1.squarespace.com/static/52af4c8ae4b09df32bf4b041/t/541c4a3ee4b0381477 9e2228/1411140487595/Role+Physical+Environ+in+the+21st+Century+Hospital_0.pdf

Väänänen, P. (2009). Testing of Biodegradable Bone Fixation Implants. Kuopio University.

Vanderson. (2010). OneMedPlace - Sentinel http://www.onemedplace.com/blog/wpcontent/uploads/2010/09/footprint.jpg. Retrieved from http://www.onemedplace.com/blog/archives/6286

Vandrovcová, M., \& Bacakova, L. (2011). Adhesion, growth and differentiation of osteoblasts on surface-modified materials developed for bone implants. Physiol Res, 60(3), 403-417.

Wagner, K. L. (Filed). Bone graft materials containing calcium phosphate and chitosan. Retrieved from http://www.google.com/patents/US20140271914

Wheelen, T. L., \& Hunger, J. D. (2006). Strategic management and business policy: concepts and cases (10th ed). Upper Saddle River, NJ: Pearson Prentice Hall.

World Health Organization. (2014). World health statistics 2014. 
Wu, Y., Cheng, C., Yao, J., Chen, X., \& Shao, Z. (2011). Crystallization of Calcium Carbonate on Chitosan Substrates in the Presence of Regenerated Silk Fibroin. Langmuir, 27(6), 28042810.

Yarbrough, A. K., \& Smith, T. B. (2007). Technology Acceptance among Physicians: A New Take on TAM. Medical Care Research and Review, 64(6), 650-672. doi:10.1177/1077558707305942

Yildirimer, L., \& Seifalian, A. M. (2014). Three-dimensional biomaterial degradation - Material choice, design and extrinsic factor considerations. Biotechnology Advances, 32(5), 984-999. Yin, Y., Ye, F., Cui, J., Zhang, F., Li, X., \& Yao, K. (2003). Preparation and characterization of macroporous chitosan-gelatin/ $\beta$-tricalcium phosphate composite scaffolds for bone tissue engineering. Journal of Biomedical Materials Research Part A, 67A(3), 844-855.

Zamparo, E. (2011, November 9). The costs of a microCT scan. Retrieved from http://microctworld.net/the-costs-of-a-microct-scan/

Zhang, S., \& Gonsalves, K. E. (1995). Synthesis of calcium carbonate-chitosan composites via biomimetic processing. Journal of Applied Polymer Science, 56(6), 687-695. doi:10.1002/app.1995.070560606

Zhou, C., Ma, L., Li, W., \& Yao, D. (2011). Fabrication of tissue engineering scaffolds through solidstate foaming of immiscible polymer blends. Biofabrication, 3(4), 045003.

Zikmund, W. G., Ward, S., Lowe, B., Winzar, H., \& Babin, B. J. (2010). Marketing research. South Melbourne, Vic.: Cangage Learning. 